\title{
New ISO standards for hearing protectors (A)
}

\author{
Poulsen, Torben
}

Published in:

Acoustical Society of America. Journal

Publication date:

2000

Document Version

Publisher's PDF, also known as Version of record

Link back to DTU Orbit

Citation (APA):

Poulsen, T. (2000). New ISO standards for hearing protectors (A). Acoustical Society of America. Journal, 108(5), 2619-2619.

\section{General rights}

Copyright and moral rights for the publications made accessible in the public portal are retained by the authors and/or other copyright owners and it is a condition of accessing publications that users recognise and abide by the legal requirements associated with these rights.

- Users may download and print one copy of any publication from the public portal for the purpose of private study or research.

- You may not further distribute the material or use it for any profit-making activity or commercial gain

- You may freely distribute the URL identifying the publication in the public portal

If you believe that this document breaches copyright please contact us providing details, and we will remove access to the work immediately and investigate your claim. 


\title{
Session 4aAA
}

\section{Architectural Acoustics: Theme Park Acoustics}

\author{
David E. Marsh, Chair \\ Pelton Marsh Kinsella, 1420 West Mockingbird Lane, \#400, Dallas, Texas 75247-4932 \\ Chair's Introduction-8:25 \\ Invited Papers
}

8:30

4aAA1. Challenges and more in the acoustic design of an attraction. Neil A. Shaw and Ray Van den Broeck (Menlo Sci. Acoust., Inc., P.O. Box 1610, Topanga, CA 90290-1610)

Building a theme park from scratch involves not only providing a "theme" for the attractions contained therein, but also integrating the acoustic control and enhancement measures so that they work with, and not against, the goal of the attraction, which is the suspension of disbelief, at least for the age group to which the attraction is designed. The challenges encountered by the authors, the analysis of the same, as well as the proposed solutions, in the acoustic design for an enclosed attraction that includes hands-on interactive activities, live performances, and a roller coaster will be reviewed. Field work to determine potential mitigation paths will also be discussed.

\section{9:00}

4aAA2. Creativity in theme park acoustics. Marshall Long (Marshall Long Acoustics, 13636 Riverside Dr., Sherman Oaks, CA 91423, m_long@pacificnet.net)

Theme parks are places where the entire environment is a theater in which the guests are active participants. As such it emphasizes creativity of concept and design, including acoustical design. Creative solutions have been found for acoustical problems including innovative uses of materials, mechanical and sound systems. A number of examples are presented from the author's 20-year experience with theme park acoustics illustrating examples of creative use of scientific principles.

\section{9:30}

4aAA3. Knott's Camp Snoopy at the Mall of America: A theme park under glass, revisited. Steven J. Thorburn (2867 Grove Way, Castro Valley, CA 94546, SJT@TA-Inc.com)

Over ten years ago, the design of a special theme park was born. The plans for Knott's Camp Snoopy at the Mall of America in Minneapolis, Minnesota would eventually grow into a popular tourist destination, but there were initial concerns about acoustics. The owners were familiar with the high noise levels of other indoor amusement parks and wanted to avoid that same situation. Having just completed the West Edmonton Mall, where many complaints were received about noise levels and guest visits were very short, it was very important to the owners that the design would prevent problems associated with noise. To do this, an acoustical consulting engineering firm was brought in. In this paper, the original acoustical design goals and solutions will be reviewed and compared against the final constructed project and its success over the first decade.

\section{0:00}

4aAA4. Community noise from theme parks. Ted N. Carnes (Pelton Marsh Kinsella, 1420 W. Mockingbird Ln., Ste. 400, Dallas, TX 75247)

Theme and amusement parks are made to generate fun and excitement. As part of the guest experience a great amount of sound energy is often generated and it may be perceived as noise in the surrounding community. Defining outdoor noise criteria for the parks is often done for you by the local municipality zoning and building officials. Thus, one can use sound-modeling techniques to determine potential problems before the parks are built or expanded. While noise control of sound levels at the source is always desirable, there are many sources that defy their use. Thus, control of sound levels along the sound path is all that is often available. All of these concerns about theme parks will be addressed along with some suggestions on how to minimize community noise impacts. Some actual project examples will be discussed with the architectural acoustics aspects emphasized.

10:30

4aAA5. The impact of propagation anomalies on noise emissions from entertainment facilities. Robert Bronsdon (Walt Disney Imagineering, 1401 Flower St., Glendale, CA 91221)

Most measurements made on environmental noise sources are done whenever it is convenient without any real consideration to the effects of environmental factors such as wind and temperature gradients. Predictions are typically done assuming that the atmospheric conditions are stable and benign. When the predictions and test results are compared and do not match, a generalization is given: "It 
must have been caused by some type of atmospheric condition." A computational Gaussian beam program that accounts for the effects of wind and temperature inversions was presented at Inter-noise 98 in New Zealand and is now currently a part of the SoundPLAN program for evaluating environmental noise. That program is designed primarily to deal with temperature inversions and wind that is blowing from the source to the receiver, but it does provide insight into upwind propagation. This paper explains how upwind propagation of sound in temperature inversion conditions can impact noise receivers in urban settings.

\title{
11:00
}

4aAA6. Noise abatement strategies and modeling approaches for outdoor attractions at theme parks. Christopher W. Menge (Harris Miller Miller \& Hanson, Inc., 15 New England Executive Park, Burlington, MA 01803, cmenge@hmmh.com)

Recent experience in noise abatement approaches and results for a new theme park are presented. Significant contributions from different source components on roller coasters lead to different abatement strategies taken for each. Measured source data will be presented. The relative performance of abatement applied separately to the source and sound path will be discussed, as well as the effects of source directivity. Source and propagation modeling approaches are presented, including how a customized version of a recently developed national noise model for highway traffic was applied for roller coasters and other sources.

\section{Session $4 \mathbf{a A B}$}

\section{Animal Bioacoustics: Instrumentation for Animal Bioacoustics Monitoring and Measurement}

\author{
William C. Burgess, Chair \\ Greeneridge Sciences, 1411 Firestone Road, Goleta, California 93117
}

Chair's Introduction-7:25

Invited Papers

$7: 30$

4aAB1. Reflector microphones for field recording of natural sounds. George W. Swenson, Jr. (Dept. of Elec. and Computer Eng., Univ. of Illinois at Urbana-Champaign, 1308 W. Main St., Urbana, IL 61801, gswenson@uiuc.edu)

A directional microphone system for field recording of sounds in the air, for example, bird song or other animal activity, usually involves either a parabolic reflector to focus the sound waves on the microphone (transducer) element, or a linear array of transducers so phased as to respond preferentially to sounds from one directional sector. The latter system (the so-called "shotgun" microphone) can be analyzed in a fairly straightforward manner. The reflector system, however, involving as it does a structure comparable to a wavelength in linear dimension, is not susceptible to the conventional approximate methods of computation. Recently developed computational techniques now permit exact calculation of the directional responses of small reflectors. One result is a proposal for a very economical and effective system involving a plane reflector. No directional microphone can, in practice, reproduce sounds with fidelity to the sounds as emitted by the source.

4aAB2. Portable instrumentation for the recording, analysis, and playback of infrasonic animal vocalizations. Elizabeth von Muggenthaler (Fauna Commun. Res. Inst., P.O. Box 1126, Hillsborough, NC 27278)

Several bioacoustic experiments required an affordable system capable of field recording, real-time analysis, signal editing, and real-time playback of infrasonic animal vocalizations. The synthesis of several types of recording devices and signal processing software has resulted in the capacity to record infrasound and perform analysis even during rain, heat, and high humidity. Small portable microphones and recorders can record in the field from $3 \mathrm{~Hz}$ to $22 \mathrm{kHz}$. On-site analysis including real-time FFT, color spectrographic function, filtering, cross-correlation, and other functions can be facilitated with the use of any portable computer with 92 Mbytes RAM. Signal editing, including frequency, amplitude, and cut/paste can be accomplished with readily available music signal processing software. Real-time field playback from $10 \mathrm{~Hz}$ to $45 \mathrm{kHz}$ can be acheived by using portable car audio speakers. This system was used for the real-time analysis of elephant vocalizations, which were edited and played back immediately during recording sessions. It has also been used in field research involving tiger, binturong, rhinoceros, and giraffe. Tigers and elephants appear to respond behaviorally to real-time playback of original and edited vocalizations. [Work supported by National Instruments, Momentum Data Systems, and Sonic Foundry.] 
4aAB3. Animal sound analysis and playback software: Building a software tool useful to field researchers. John M. Burt (Cornell Lab of Ornithology, 159 Sapsucker Woods Rd., Ithaca, NY 14850)

Many researchers would like to conduct sound-playback experiments with the level of control potentially available using a computer, and yet there are few programs specifically designed for computer-based playback. The lack of PC playback software led to the development of a WINDOwS 98 application called SYRINX, which is specifically designed for both sound analysis and playback in the field. The goal was to provide a host of playback features that would allow researchers to conduct sophisticated field playback experiments using a sound card-equipped laptop PC and a portable speaker. SYRINX can display multiple sound files as playable spectrograms, has a small suite of tools for editing sounds, displays a real-time scrolling spectrogram of audio input, allows users to log behavioral events, and has a number of other features useful for playback research. The talk will focus on the field-research-driven design goals for the SYRINX project, how the program was implemented, what signal-processing tools and features were thought to be necessary for field researchers, what new features may be implemented in the future, and the challenges involved in developing a researcher- and field-friendly playback program.

8:30

4aAB4. Geographically distributed acoustical monitoring of migrating birds. Harold Mills (Bioacoustics Res. Prog., Cornell Lab. of Ornithology, 159 Sapsucker Woods Rd., Ithaca, NY 14850, hgm1@ cornell.edu)

A geographically distributed system of largely automated acoustical monitoring stations was developed to monitor the migration of small passerine birds. The targeted species, wood warblers and sparrows, migrate at night and produce short (about $120 \mathrm{~ms}$ at the longest), high-pitched (between roughly 6 and $10 \mathrm{kHz}$ ) calls as they fly, often audible from the ground. The monitoring system consisted of ten stations, located in New Jersey, Maryland, Pennsylvania, and New York, and a central data repository located at the Cornell Lab of Ornithology. Each station comprised an outdoor, weatherproof microphone connected to a volunteer's home computer. The computer automatically ran acoustic transient detection software each night that listened continuously to the microphone signal and extracted all transients matching certain criteria, each to its own audio file. The detected transients were uploaded by the volunteer each morning via the Internet to the central repository for classification and archival. Results, including spectrograms of all detected transients and bird call counts, were displayed on a public web site updated daily. [Work supported by EPA.]

\section{8:50}

4aAB5. A compact digital recording system for autonomous bioacoustic monitoring. Thomas A. Calupca, Kurt M. Fristrup, and Christopher W. Clark (Bioacoustics Res. Prog., Cornell Lab. of Ornithology, 159 Sapsucker Woods Rd., Ithaca, NY 14850)

A digital recording system with several months' endurance was designed to monitor biological sounds. The system combined off-the-shelf components (microcontrollers, notebook hard drives) with an IDE interface designed for this purpose. The present storage capacity of the system is 25 Gbytes, with a maximum sampling rate of $50 \mathrm{kHz}$ at a resolution of 12 bits. Power consumption during typical long-term continuous recording averages 250-500 milliwatts, and its physical dimensions are approximately $3 \times 7$ $\times 10 \mathrm{~cm}$, including the hard drive. Hardware and software support for GPS clock synchronization enables correction of clock drift in the system, as well as precise synchronization of multiple units in an array. This unit has been extensively utilized in bottom-moored recording buoys, called "popups." Popups include an acoustic communication system that receives and acknowledges commands from a surface support vessel, and an electronically controlled galvanic release. Everything is housed in a single 17-in. glass pressure housing. More recently, these systems have been used to monitor low-frequency terrestrial signals in studies of forest and savannah elephant populations. An upcoming project will drift these systems over inaccessible habitats using helium balloons.

9:10

4aAB6. An autonomous acoustic recorder using a directional sensor for locating calling bowhead whales. Robert G. Norman and Charles R. Greene, Jr. (Greeneridge Sci., Inc., 1411 Firestone Rd., Goleta, CA 93117)

A new directional acoustic recorder has been developed to assess the movement and distribution of vocalizing marine mammals. Called DASAR (Directional Autonomous Seafloor Acoustic Recorder), the instrument incorporates an omnidirectional and two orthogonal horizontal sensors from a DIFAR (DIrectional Frequency and Recording) sonobuoy, as well as a magnetic compass. With appropriate processing, the horizontal directions can be computed for received sounds. The acoustic bandwidth is $1000 \mathrm{~Hz}$, appropriate for bowhead calls, and recording is continuous for 45 days. DASARs were deployed in a hexagonal array seaward of an artificial gravel island recently constructed for oil and gas production northwest of Prudhoe Bay, Alaska. Operation was during the fall migration of bowhead whales past the island. The objective was to relate calling-whale locations to the character and levels of sounds emanating from the island and from nearby tugs and barges. [Work supported by BP Exploration (Alaska).]

\section{9:30}

4aAB7. A digital acoustic recording tag for measuring the response of marine mammals to sound. Mark Johnson, Peter Tyack, Douglas Nowacek (Woods Hole Oceanogr. Inst., Woods Hole, MA 02543, majohnson@whoi.edu), and Alex Shorter (Univ. of Colorado, Boulder, CO 80302)

There is growing concern about the effect on marine mammals of underwater sound sources such as vessels, industrial equipment, and sonar. In order to quantify the reaction of these animals to sound, controlled exposure experiments have been attempted using surface observations or vocal monitoring to estimate response. However, the short surface time of most marine mammals, and their often unknown vocalization rates, limit the effectiveness of such experiments. To address these problems, a small digital recording tag, the DTAG, has been developed capable of simultaneously sampling the acoustic environment of the host animal, together with its orientation, heading, and depth. The tag has a 400-Mbyte memory array sufficient to record audio and sensor signals for several hours. 
The tag is encapsulated in a plastic resin and can operate at a water depth up to $3000 \mathrm{~m}$. Programing and data off-load are accomplished with an infrared communications link to a personal computer. The DTAG has been deployed on northern right whales and sperm whales using suction cup attachments. Delivery was by means of a cantilevered pole from a small boat. The resulting data indicate a number of behavioral response metrics as well as new results on dive behavior and vocalization rates.

9:50

4aAB8. The bioacoustic probe: A general-purpose acoustic recording tag. William C. Burgess (Greeneridge Sci., Inc.)

Recent research has demonstrated the utility of acoustic recording tags to assess the exposure and response of marine wildlife to sound [e.g., W. C. Burgess et al., Deep-Sea Res., Part II 45, 1327-1351 (1998)]. The experimental nature of these tags, however, has limited their adoption by the wider bioacoustical community. To enable broader use of acoustic recording tag technology, a new general-purpose tag is being developed. Miniaturization to a cylinder approximately $3 \mathrm{~cm}$ in diameter by $16 \mathrm{~cm}$ in length will allow application with a variety of species and attachment methods. Initial versions of the device will sample acoustics with 16-bit resolution at bandwidths up to $14 \mathrm{kHz}$, as well as temperature and depth with 12-bit resolution. Longevity will depend on the choice of sampling schedule; constant acoustic sampling at $2 \mathrm{kHz}$ will fill the $288 \mathrm{MB}$ solid-state flash disk in $21 \mathrm{~h}$, but this lifetime can be extended by reducing resolution or by recording only during times of interest. Results from initial test deployments on free-ranging marine mammals are expected in late fall 2000. [Work supported by ONR.]

\section{0:10-10:20 Break}

\section{Contributed Papers}

10:20

4aAB9. Echolocation signals of wild white beaked dolphins measured with a four-hydrophone short base line array in real-time. Whitlow W. L. Au (Hawaii Inst. of Marine Biol., P.O. Box 1106, Kailua, HI 96734), Marianne H. Rasmussen, and Lee Miller (Ctr. for Sound Commun., Odense Univ., Odense, Denmark)

The echolocation signals of Lagenorhynchus albirostris were measured in the waters off Keflavik, Icelend during 22-26 August 1998. A fourhydrophone array was used with each hydrophone located at the tip of an equilateral triangle and one located at the center of the triangle. Each of the outer three hydrophones was spaced $0.61 \mathrm{~m}$ from the center hydrophone and the separation angle between outer hydrophones was $120 \mathrm{deg}$. An underwater video camera was attached $10 \mathrm{~cm}$ from the center hydrophone. The array was mounted on a long pole that was lowered to a depth of about $2 \mathrm{~m}$ from the side of a 10-m diesel driven boat. The hydrophones were connected to two GAGA-1210 data acquisition boards operating at a $500-\mathrm{kHz}$ sample rate. The computer clock was synchronized with the clock on portable video recorder. Two-hundred pretrigger and 200 posttrigger points per channel with four channels of data were acquired simultaneously and stored in the memory of a transportable computer. The results indicate that the white beaked dolphins emitted high-frequency clicks with peak frequencies close to $120 \mathrm{kHz}$. The maximum source level of the echolocation signals was $219 \mathrm{~dB}$ and the source levels increased with range from the array.

\section{0:35}

4aAB10. Miniature wildlife telemetry transmitter equipped with a microphone. Larry Pater (USA CERL, 2902 Farber Dr., Champaign, IL 61821), William Cochran (Univ. of Illinois), and George Swenson, Jr. (Univ. of Illinois)

A miniature microphone was integrated into a telemetry transmitter package with a total weight of 1.2 grams (operating life of 7 days) or 2.0 grams (21-day life). To obtain meaningful acoustical information, the radio transmitter is continuous transmission, rather than the pulsed type typically used for wildlife telemetry. It is sensitive enough to pick up bird songs at a distance of over $30 \mathrm{~m}$ and small airplane engine sounds at several kilometers. This device is useful for remote monitoring in inaccessible locations and to avoid the intrusive impact of direct observation by humans. It can be used to document acoustical environment and auditory stimuli and to monitor vocalizations and behavior of the animal on which the transmitter package is mounted. Applications include studying behavior and responses to disturbance. The transmitter has been successfully used to monitor bird calls and wing-beat patterns during various types of activity, including foraging and nighttime migration flights.
10:50

4aAB11. Use of a radio microphone array to study banded-wren song interactions at the neighborhood level. John M. Burt (Cornell Lab. of Ornithology, 159 Sapsucker Woods Rd., Ithaca, NY 14850)

An array of distributed microphones was used to locate and identify singing banded wrens in Santa Rosa National Park, Costa Rica. Wrens were recorded both during natural singing interactions and during song playback experiments. The array consisted of 11 weatherproofed radiomicrophones distributed among neighborhoods of up to seven bandedwren territories, with a centrally located radio receiver/recording station. Audio from the radio receivers was stored to a laptop computer using a multichannel data-acquisition card and custom recording software. Singing birds were located after recording using software developed at the Cornell Lab of Ornithology's Bioacoustics Research Program. The system's durability and flexibility enabled several different array configurations to be tested at two field sites under a range of recording conditions. Preliminary results suggest that this system is a powerful and flexible tool for studying the dynamics of vocal interactions across entire neighborhoods of singing birds.

\section{1:05}

4aAB12. Remote infrared acoustically triggered video monitoring unit. Robert Ambrose (U.S. Fish and Wildlife Service, 1011 E. Tudor Rd., Anchorage, AK 99503), Michael Donaldson (Far North Aquatics, Box 61264, Fairbanks, AK 99706), Timothy Lavallee, and Troy Andersen (Geo-Marine, Inc., Newport News, VA 23606)

A remotely operable, infrared system for recording digital video and audio information immediately before, during, and after an acoustical event was developed. The primary application of this system to date has been the recording and documentation of effects of noise on wildlife, including Peregrine falcons (Falco peregrinus), Mexican spotted owls (Strix occidentalis lucida), and brown pelicans (Pelecanus occidentalis). The system triggers, records, and stores digital video and audio data on an IBM compatible computer running wIN9X. Serial communication with a Larson Davis sound-level meter allows a field video camera to store video and audible sound data on the computer. The need for field crew collection of bioacoustical effects data at sites with video acquisition systems is minimized. The video information reduces observation or interpretation bias in the data collection procedures. The systems can be maintained from remote locations using cellular modem technology and can be operated without being visited for extended periods. It is low powered and can be operated by a system of batteries and solar panels for indefinite field deployment. With the correct lens the system is capable of seeing in complete darkness up to $25 \mathrm{ft}$ or as far away as $200 \mathrm{ft}$ in the daytime. 
$11: 20$

4aAB13. Programmable DSP datalogger for animal bioacoustics monitoring. David A. Mann and Timothy J. Tucker (Tucker-Davis Technologies, 4637 NW 6th St., Gainesville, FL 32609)

Two hardware modules for animal bioacoustics monitoring based on the Analog Devices Sharc DSP are described. The RP2 Real-Time Processor connects to a PC via the USB port and can be used for signal detection and direct logging to disk. The SD2 Smart Datalogger is a battery-powered digital datalogger. The RP2 has two channels of A/D with $100-\mathrm{kHz}$ bandwidth, two channels of D/A with $200-\mathrm{kHz}$ bandwidth, eight digital inputs, eight digital outputs, and up to 64 MB RAM. The SD2 Smart Datalogger has two channels of A/D and D/A with a $25-\mathrm{kHz}$ bandwidth on each channel, two digital I/O channels, and up to 64 MB RAM. The datalogger weighs 2 ounces and its dimensions are $5.6 \times 7.6$ $\times 1.8 \mathrm{~cm}^{3}$. DSP programming for both devices is accomplished through the use of a drag-and-drop interface for configuring detection circuits using a library of over 120 DSP components including digital filters, signal detectors, and memory operations. The use of the RP2 for detecting and recording animal sounds in the field will be described where sounds in given frequency bands are detected, time-stamped, and saved to memory or hard disk. [Work supported by NIH.]

\section{1:35}

4aAB14. The development of a compact instrument for the measurement of biological sea noise. Alec J. Duncan (Ctr. for Marine Sci. and Technol., Curtin Univ. of Technol., Bentley, Western Australia), Douglas H. Cato (Defence Sci. and Technol. Organisation, Pyrmont, NSW 2009, Australia), Frank Thomas, and Robert D. McCauley (Curtin Univ. of Technol., Bentley, Western Australia)

This paper describes a compact, low-power instrument that has been developed to monitor biological sound sources and other sea noise over extended periods in remote locations. The instrument is based on a low power microcontroller and was designed to provide flexibility in specify- ing sampling rates and sampling schedules so that the utilization of battery power and disk space could be optimized for any given application. In its basic configuration the signals from two hydrophones are sampled and stored on a 12-GByte hard disk drive. Multiple drives can be installed if greater capacity is required. The electronics fits inside a 100 -mm i.d. underwater housing resulting in a package that is easy to transport and deploy. Further details of the instrument design will be given, together with the results of deployments off the Western Australian coast where it has been used to monitor the passage of migrating humback whales.

11:50

4aAB15. Detection of harbor porpoises and white whales (Beluga) sound using the high frequency bioacoustics and their echo structures. Erhan Mutlu (Inst. of Marine Sci., METU, P.O. Box 28 Erdemli, 33731 Turkey, mutlu@ims.metu.edu.tr)

Acquisition of sounds emitted by marine mammals (porpoise and beluga) living in the Black Sea was made using active echosounder (BioSonics Model 102) with down-looking 120- and 200-kHz transducers. The sounds of the mammals displayed different structures not similar to each other. The individual amplitude of the porpoise sound was significantly discriminated and varied between -55 and $-62 \mathrm{~dB}$ at $200 \mathrm{kHz}$. The echoes were characterized with decreasing amplitude from the deep water to the surface. It looked like vertical lineations. The sound scattering was fluctuated in a range of $10-20 \mathrm{~dB}$. Sound emitted by beluga displayed a structure like rakes of a comb. The echo was captured first very weak at $120 \mathrm{kHz}$ and then strong at $200 \mathrm{kHz}$. The volume scattering changed within a range of 60 and $-70 \mathrm{~dB}$ at $200 \mathrm{kHz}$. While the whale was close to the transducer, it emitted the sound every $300 \mathrm{~s}$. It sometimes gave sound in different patterns from some reason. As we got away from the whale, while on the way, we detected the sound at $10 \mathrm{~s}$. Each lineation of the comb-rake structure appeared in a certain time delay and the strength decreased toward the surface.

\title{
Session $4 \mathrm{aAO}$
}

\section{Acoustical Oceanography: Acoustical Oceanography and Satellite Remote Sensing}

\author{
Daniel L. Hutt, Chair \\ Defence Research Establishment Atlantic, P.O. Box 1012, Dartmouth, Nova Scotia B2Y 3Z7, Canada
}

Chair's Introduction-7:50

Invited Papers

\begin{abstract}
8:00
4aAO1. Estimation of subsurface ocean density structure using remote sensing and data assimilation. Josko Bobanovic and Keith Thompson (Dept. of Oceanogr., Dalhousie Univ., Halifax, NS B3H 4J1, Canada, josko@phys.ocean.dal.ca)

Recently, researchers from Dalhousie University, The Royal Military College, and the Defence Research Establishment Atlantic started work on estimating water density, and eventually underwater acoustical properties, from in situ and remotely sensed data. This requires the development of assimilation schemes to project vertically into the ocean interior the surface information gathered by remote sensing. Initially, we are concentrating on inferring the seasonal distributions of temperature and salinity on the continental shelf. We are experimenting with new ways of assimilating such data into fully nonlinear, baroclinic models using the incremental approach. In a nutshell, the approach uses two parallel ocean models: a complex model to be fit to the data, and a simple model with known adjoint that is used to correct the control variables of the complex model. The result is a set of seasonal fields that is consistent with the observed data, and dynamical constraints imposed by the model, within prescribed errors bars. Our long-term objective is to develop practical schemes for assimilating time sequences of remotely sensed (e.g., SST, ocean color, altimetry, HF radar) data into any ocean model. Progress will be reviewed.
\end{abstract}


4aAO2. Using MODAS to derive subsurface sound speed estimates from satellite observations. Charlie N. Barron, Daniel N. Fox (Naval Res. Lab., Oceanogr. Div., Stennis Space Center, MS 39529), and Eleanor Holmes (IPD/A\&T/Anteon, Arlington, VA 22202)

The Modular Ocean Data Assimilation System (MODAS) provides a set of tools for analysis and manipulation of ocean data. One of its functions is the estimation of subsurface temperature and salinity fields from satellite observations of height deviation and temperature. A two-dimensional optimal interpolation of the satellite data produces fields of surface height anomaly and temperature. These data are provided to the MODAS synthetic profile routine to predict subsurface temperature and salinity fields compatible with the surface observations and local climatological statistics. Available in situ measurements may be assimilated at this stage. A sound speed field calculated from the temperature and salinity estimates provides the basis for additional acoustical applications. MODAS products, evaluated by comparison with finely sampled surveys, show significant improvement over estimates from static climatologies. [This work was funded by the Space and Naval Warfare Systems Command (SPAWAR) and the ONR through the Advanced Data Fusion Center Support and the On-Scene Tactical Ocean Forecast Capability projects (SPAWAR, Program Element 603207N) and the MODAS Improvements and the Prediction of Coastal Buoyancy Jets projects (ONR, Program Element 602435N).]

9:00

4aAO3. MEANS: Multiscale environmental assessment network studies. Henrik Schmidt (MIT, 77 Massachusetts Ave., Cambridge, MA 02139), Allan Robinson (Harvard Univ., Cambridge, MA 02139), Lakshmi Kantha (Univ. of Colorado, Boulder, CO), and Jurgen Sellschopp (SACLANT Undersea Res. Ctr., La Spezia, Italy)

The small-scale coastal acoustic environment is extremely difficult to predict and forecast. The very shallow near-shore region, with water depths of up to a few tens of meters, is particularly challenging due to the variability of the critical seabed properties due to sediment transport processes, biological processes, and changes in small-scale bathymetry due to wave action, with strong implications for the high-frequency reverberation characteristics in particular. The potential of using small autonomous underwater vehicles (AUV) with high dependency on acoustic systems in such environments has led to an increased need for forecasting the acoustic characteristics. The coastal ocean environment is a result of a very complex dynamical system with strong coupling among different scales. To investigate the predictability of this process, the MEANS effort implements a nested forecasting framework for the small-scale oceanography in Procchio Bay, Elba during the GOATS'2000 AUV technology experiment in Sep.-Oct. 2000. A local high-resolution model is constrained by assimilating oceanographic data from AUVs and moorings, and nested within a regional Ligurian Sea model and a basin scale circulation model, both constrained by surface ship measurements and satellite remote sensing data. The nested observation and modeling framework will be described, and preliminary results will be presented and discussed. [Work supported by ONR and SACLANT.]

9:30

4aAO4. The dependence of low-frequency underwater surface scattering on remotely sensed oceanographic variables. Roger C. Gauss (Naval Res. Lab., Washington, DC 20375-5350, roger.gauss@nrl.navy.mil), Eric I. Thorsos, Frank S. Henyey (Univ. of Washington, Seattle, WA 98105-6698), and Joseph M. Fialkowski (Planning Systems, Inc., McLean, VA 22102-3304)

Recent low-frequency $(<1500 \mathrm{~Hz})$ underwater acoustic measurements have demonstrated that scattering processes at the air-sea interface depend primarily on the wind speed and the surface wave spectrum. The scattering strength from the rough interface is proportional to the spectral density at the Bragg wavelength with modifications due to tilt and modulation by longer waves. These modifications are accounted for in the small slope approximation. When wave breaking becomes significant, rough interface scattering is augmented by bubble cloud scattering, which depends primarily on wind speed. In this regime, bubble cloud scattering dominates at low grazing angles, and rough interface scattering dominates at high grazing angles. A physics-based empirical model is used to describe bubble scattering. The mean-frequency-shift characteristics of acoustic signals scattered from both the moving sea surface and bubble clouds have been successfully modeled given the 2-D surface wave spectrum. These scattering-strength and frequencyshift models are used to explore the sensitivity of low-frequency scattering to environmental variables obtainable by remote sensing. [Work supported by ONR.]

10:00

4aAO5. The estimation of acoustic properties of internal waves using synthetic aperture radar (SAR). Justin Small and Mark Prior (Defence Evaluation Res. Agency, Winfrith Technol. Ctr., DT2 8XJ, UK)

This paper looks at some methods relevant to the assessment of the acoustic properties of internal waves from satellite data. The currents in these internal waves strain the sea surface leading to signatures, which can be imaged by instruments such as radar, photography, and SAR. Two lines of approach for estimating internal wave amplitudes from SAR will be illustrated. The first approach uses length-scale information on SAR (separation of waves, width of waves), combined with some knowledge of the stratification (from a database or survey) and a solitary internal wave or undular bore model. The second approach uses radar imaging models to investigate the strength of current which would lead to the observed signatures in the given wind conditions. One important acoustic property of internal waves is the influence on surface duct propagation due to the large measured excursions of the mixed layer. Examples will be shown of the effects on surface duct propagation for different frequencies and scenarios using experimental data taken (1) off the northwest of the UK; (2) in the central Bay of Biscay; and (3) on the Portuguese coast.

10:30-10:45 Break 


\title{
Contributed Papers
}

10:45

4aAO6. Horizontal refraction modal tomography (HRMT) for ocean front and ocean current monitoring. E. C. Shang and Y. Y. Wang (CIRES, Univ. of Colorado/NOAA/Environ. Technol. Lab., 325 Broadway, Boulder, CO 80303)

It has been pointed out by Voronovich and Shang [IEEE J. Ocean. Eng. 24, 224-231 (1999)], that the HRMT has the ability of reducing the mode-coupling effect. In this paper, two examples of the application of HRMT in strong mode-coupling environment are discussed: (1) Polar front, and (2) transverse current in Fram Strait. In both cases, strong mode coupling takes place for the used frequency and mode number. Due to the modal phase difference is measured by two close paths, the modecoupling effect can be significantly reduced. The results of the retrieved frontal parameters and the transverse component of the current based on adiabatic theory are pretty good even without any iteration. For the Polar front, the horizontal refraction angle is estimated. The parameters that can be retrieved by using HRMT are: the normal direction of the front, the center location of the front, and the sound speed profile difference of the two water masses. The interaction of those acoustically estimated parameters with satellite remote sensing is discussed. [Work supported by NOAA and ONR.]

\section{1:00}

4aAO7. A comparison of acoustic and TOPEX/POSEIDON altimetric data in the Kuroshio Extension region during summer 1997. Gang Yuan, Humio Mitsudera (IPRC-SOEST, Univ. of Hawaii, 2525 Correa Rd., Honolulu, HI 96822-2219), Iwao Nakano, Hidetoshi Fujimori, Yasushi Yoshikawa, and Toshiaki Nakamura (Japan Marine Sci. and Tech. Ctr., Yokosuka, 237-0061 Japan)

Approximately two months of data from a five-transceiver acoustic tomography array in the Kuroshio Extension (KE) in the summer of 1997 are used for comparing with the TOPEX/POSEIDON (T/P) altimeter measurements. The acoustic data show that travel time (TT) variations are very consistent with contemporaneous variations of sea surface height anomalies (SSHA) as in the T/P in the KE front region, but have significant differences in the KE recirculation region. This difference is assumed to be due to the fact that the altimeter might not pick up the signal associated with the variations occurring below the seasonal thermocline. The inversions from a new approach show that the temperature in the surface layer $(0-100 \mathrm{~m})$ had been warming up $1.0{ }^{\circ} \mathrm{C}$ during the experimental period, but cooling down about $0.2{ }^{\circ} \mathrm{C}$ in the subsurface layer (100-1500 $\mathrm{m})$. This temperature warming up in the surface layer derived from acoustic data is excellent, consistent with the steric height change determined from net heat flux data of the NCEP/NCAR. Zonal range and depth averaged (barotropic) westward-flowing current velocity determined from reciprocal acoustic TTs in the recirculation region is about $5 \mathrm{~cm} / \mathrm{s}$; it is comparable with the surface geostrophic current velocity determined from the SSHA of the T/P. Acoustic tomography complements the altimetry.

\section{1:15}

4aA08. Application of space-based synthetic aperture radar imagery for estimating underwater ambient noise. Daniel L. Hutt (Defence Res. Establishment Atlantic, P.O. Box 1012, Dartmouth, NS B2Y 3Z7, Canada,daniel.hutt@drea.dnd.ca)

Synthetic aperture radar (SAR) imagery of the ocean surface provided by the Radarsat satellite can provide estimates of surface waves, winds, and shipping density. These are the parameters which are mainly responsible for underwater acoustical ambient noise. The remotely sensed surface conditions can be combined with knowledge of the acoustical propagation environment and geoacoustic properties of the bottom to estimate the ambient noise field. Although difficulties exist with SAR-derived wind fields and ship detection, these are balanced by the wide area coverage and high spatial resolution of the SAR sensor. We review space-based SAR capabilities relevant to ambient noise estimation and compare Radarsatderived wind fields to in situ measurements for two Radarsat passes over the Atlantic Ocean. Ambient noise levels measured during the overpasses compare favorably with those estimated using the Radarsat wind data

THURSDAY MORNING, 7 DECEMBER 2000

PACIFIC SALONS A, B AND C, 7:55 TO 11:00 A.M.

\section{Session 4aEA}

\section{Engineering Acoustics: Acoustic Noise Characterization and Mitigation}

\author{
James O. Buntin, Chair \\ Brown-Buntin Associates, Inc., 7996 California Avenue, Suite A, Fair Oaks, California 95628
}

Chair's Introduction-7:55

\section{Contributed Papers}

8:00

4aEA1. Gas turbine noise mitigation for a residential development. Jim Buntin (Brown-Buntin Associates, Inc., 7996 California Ave., Ste. A, Fair Oaks, CA 95628)

A residential development was proposed adjacent to a gas turbine electrical power production peaking facility. To determine compliance with local standards, noise levels and frequency content were measured at reference locations under summer and winter conditions. The Environ. Noise
Model (ENM) was calibrated on the basis of the reference noise level data, and noise measurement results at other locations were compared to the ENM predictions. The measurement results were also used to determine the effective noise source height for calculations of shielding. The ENM was then used to predict noise levels at the development site. To evaluate methods of obtaining compliance with the standards, the ENM was used to assess the effects of berms, walls, and grading on the development site, and an enclosure for the gas turbines. Recommendations were then prepared for noise abatement and mitigation measures, including the use of an enclosure, an onsite berm, and reduction of low frequency noise. 
4aEA2. Computational investigation of wave steepening in a twodimensional duct. W. R. Erskine, A. Selamet, and P. M. Radavich (Dept. of Mech. Eng., The Ohio State Univ., Columbus, OH 43210)

The need for accurate modeling of nonlinear phenomena, such as wave steepening, in unsteady flows is important in many engineering applications. Wave steepening causes the initial sinusoidal shape of the oscillation to distort and eventually form a shock wave. Both pressure and velocity waves steepen as flow moves downstream inside a duct. A computational fluid dynamics code, which solves the compressible, unsteady, two-dimensional Navier-Stokes equations, is used to investigate this nonlinear behavior and predict the flow field in a long narrow duct. A sinusoidal inlet velocity and an anechoic termination outlet are imposed as boundary conditions. The shape and frequency content of the traveling wave are predicted at various locations inside the duct. Inlet velocity is varied to cover a range of small-to-large amplitude pressure fluctuations. Results indicate that wave steepening is enhanced as the wave travels downstream and/or as the fluctuation amplitude increases. At large enough amplitudes and/or duct lengths, a shock is produced which travels to the outlet. Regardless of the oscillation frequency of the inlet velocity, the frequency spectra reveal sound-pressure level amplitude peaks at multiples of both quarter- and half-wavelength resonances based on the length of the duct.

\section{8:30}

4aEA3. Fast acoustic power computations for structural optimization problems. John B. Fahnline (Applied Research Lab., 16 Appl. Sci., University Park, PA 16802)

Boundary element analyses in acoustics are often computationally inefficient because a large complex-valued matrix has to be solved at every frequency for which a solution is desired. This problem can be alleviated somewhat by using appropriate mesh densities for both the structural acoustic analyses, as discussed previously by the author. To achieve further enhancements, a computer program has been written to interpolate the acoustic resistance matrix over a frequency band from values calculated at sparse intervals. A preliminary analysis is performed to map out the resistance matrix as a function of frequency, requiring specification of only the frequency band of interest and the desired error tolerance. Automatic refinement is performed in regions where the resistance varies rapidly, as it does near acoustic resonances. A simple interpolation scheme is then used to compute the resistance matrix at any arbitrary frequency knowing the precomputed matrices, resulting in an order of magnitude reduction in the computation times when compared to a direct calculation. Because the acoustic analysis is independent of structural parameters, this formulation is ideal for problems where structural parameters are to be optimized without changing the radiating surface's shape. Examples are given to demonstrate the formulation's accuracy and computational efficiency.

\section{$8: 45$}

4aEA4. Lagging application with use of sound intensity. Sokcheat Monh (Brown-Buntin Associates, Inc., 7996 California Ave., Ste. A, Fair Oaks, CA 95628)

Improvements were proposed to an existing county waste water sump station. The improvements included installation of a scrubber unit used to filter odor from the sump chambers. Noise from the operation of the scrubber unit raised concerns among nearby residents. To address compliance with local standards and minimize impacts on nearby homes, sound intensity testing was used to determine which modules of the scrubber unit made significant contribution to the overall noise levels. Lagging was recommended, and it was implemented by the county. Before and after sound intensity levels of the scrubber unit demonstrated the effectiveness of lagging as a mitigation measure.
4aEA5. Computation of free-jet turbulence and exhaust-mixing noise from a turbofan engine. David B. Schein (Northrop Grumman Corp., 9L53/W6, One Hornet Way, El Segundo, CA 90245-2804 and Dept. of Mech. and Aerosp. Eng., UCLA, scheida@mail.northgrum.com) and William C. Meecham (UCLA, Los Angeles, CA)

A model presented previously [D. B. Schein, paper 3pEA3, 136th Meeting of ASA (1998)] has been extended and applied to prediction of exhaust noise from a high Reynolds number, hot exhaust flow. Simulations for a Williams Research WR19-4 turbofan engine exhaust have been performed and results compared with far-field acoustic data measured during static test runs of the engine at exhaust Mach numbers up to 0.8. The hybrid CFD/CAA approach employs a deductive subgrid scale model for small-scale compressible turbulence combined with the time-derivative form of Lighthill's source-integral acoustic analogy, containing the fluctuations represented by the time-varying stress tensor. Overall sound pressure level directivity and representative spectra are presented. Analytical results indicate generally good agreement with measured data and popular semiempirical prediction methods. Spectral analysis indicates that significantly larger computational domains and simulation times will be required to obtain low-frequency energy content radiated from aircraft engine exhausts.

9:15

4aEA6. Noise and vibration control for the Alameda Corridor project. Joel Vazquez (Parsons Eng. Sci., Inc., 100 W. Walnut St., Pasadena, CA 91124, jvazquez@parsons.com)

The Alameda Corridor project is a 20-mile railroad cargo expressline that will connect the ports of Los Angeles and Long Beach to the transcontinental rail network east of Los Angeles. Once completed, the corridor will create a fast and efficient way to move cargo through United States and overseas world markets. The midcorridor section of the project consists of a 10-mile long, 33-ft descent into a by-railway Trench. Although this Trench will be the featured portion of the midcorridor project, the construction of a by-pass track will facilitate cargo-laden freight passage while the Trench is constructed as well as provide access for local businesses after completed. On its path from the ports to downtown Los Angeles this massive construction project will traverse potential noise and vibration-sensitive areas that house single and multi-family residences, motels, schools, daycare centers, parks, and churches. One of the greatest challenges encountered with projects of this magnitude is the successful control of construction and operational noise and vibration. The presentation will focus on various noise and vibration control methods that have been implemented as part of an abatement program. The scope of this successful program includes monitoring, equipment noise certification, and impact studies.

\section{9:30-9:45 Break}

\section{9:45}

4aEA7. The multiple source feedback of supersonic jet screech, I: Theory-frequency. Alan Powell (Dept. of Mech. Eng., Univ. of Houston, Houston, TX 77204-4792)

Screech sound, generated by the interaction of jet instability waves with the spatially periodic cell structure of choked or "off-design" supersonic jets, feeds back to initiate new instability waves at the nozzle. The interaction produces a phased array of effective point sources distance $s$ apart, with a single equivalent (centroidal) source at distance $n^{\prime} s$ from the nozzle. The feedback criteria discussed at the last Meeting yield frequency $f=f_{P R} \phi$, where $f_{P R}=(U / s) \cdot 1 /(1+M)$ for perfect reinforcement at the nozzle; $U, M=$ instability wave velocity, Mach number; $\phi=(N+p+\nu$ $+I) / n^{\prime} \rightarrow 1$ with $n^{\prime} s \equiv(N+\mu+\nu) s, \nu s=$ distance of equivalent source from nearest cell end at $(N+\mu) s$ from the nozzle, $N=$ integer, $|\mu| \leqslant 1 / 2$, 
and $I=-1,0$, or +1 . Exact for any number of symmetrically disposed source strengths, numerical solutions show it to be a good approximation for arbitrary strengths and for inclusion of differing source distances (with weighted equivalent source). The mean $s$ is appropriate for unequal source spacing; but then perfect reinforcement cannot occur. With perfect reinforcement unlikely, the 1953 hypothesis that $f \rightarrow f_{P R}$ is confirmed as a simple and useful approximation.

\section{0:00}

4aEA8. The multiple source feedback of supersonic jet screech, II: Space-time diagrams and the standing wave puzzle elucidated. Alan Powell (Dept. of Mech. Eng., Univ. of Houston, Houston, TX 77204-4792)

Space-time diagrams for defined phases of the periodic instability wave and the feedback sound waves from the resultant equivalent point sources provide a simple physical picture of the multiple source feedback process, illustrated for cases of perfect reinforcement when $\phi=1$, and for $p=1 / 2$ and for $\mu= \pm 1 / 2$ when $\phi \neq 1$. They also provide a simple interpretation of the nature of the (sometimes necessary) approximation. The intersections of the maxima of the instability and sound waves form "partial standing waves", with maxima $L_{S W}=s / \phi$ apart, involving one complete cycle of the instability and sound wave. This provides a simple explanation of the puzzling standing waves observed by Westley and Woolley (1975), Rice and Taghavi (1992), and by Panda (1996, 1997), it having been speculated that they may indicate, or be fundamentally associated with, the actual sound sources. Rather, $n^{\prime} s \equiv(N+\mu+\nu) s=(N$ $+p+\nu) L_{S W}$ showing, for $\nu=0$, that $(N+p)$ standing waves (cycles) must fit in the nozzle-to-source distance, closely analogous to the $N$ 'th stage of (high-speed) edge tones. Then both $\mu$ and $p$ can be estimated from such experimental data.

\section{0:15}

4aEA9. The multiple source feedback of supersonic jet screech, III: Space-time diagrams applied to the helical mode. Alan Powell (Dept. of Mech. Eng., Univ. of Houston, Houston, TX 77204-4792)

The foregoing feedback theory applies directly to the sinuous and toroidal instability modes of supersonic jets, but needs modification for the helical $C$ mode since the acoustic feedback to the nozzle must at the axial trace velocity of the resultant helical sound field (Powell, Umeda, and Ishii, 1990). Here the space-time diagram is equivalent to an instantaneous map of the positions of the helical instability and acoustic wave on the cylindrical bounding surface of the jet, one period being replaced by one circumference. The instantaneous space-time (sound) pressure measurements of Westley and Woolley (1975) are interpreted in this way. The acoustic feedback trace velocity is found to be approximately sonic. The circumferential velocity $(=\pi f \times$ diameter source orbit diameter), in a plane normal to the jet axis is inferred to be supersonic. The phase velocity of the helical wave along the curved surface is then found to be subsonic rather than sonic as originally assumed in the tentative theory. A simple numerical simulation (including the reflection from the flange) reproduces the main features of the complex map of instantaneous pressure, including the prominent standing waves.
10:30

4aEA10. Hydrodynamic lift and drag fluctuations of a spheretheoretical model. M. S. Howe (College of Eng., Boston Univ., 110 Cummington St., Boston, MA 02215), G. C. Lauchle (Penn State Univ., State College, PA 16804), and J. Wang (The Trane Co., LaCrosse, WI 54601-7599)

A theoretical investigation is made of the unsteady lift and drag exerted on a sphere in a nominally steady high Reynolds number, incompressible flow. The net force on the sphere has previously been ascribed to fluctuations in the bound vorticity in the meridian plane normal to the force, produced by large-scale coherent structures shed into the wake. A simplified model of vortex shedding is proposed that involves coherent eddies in the form of a succession of randomly oriented vortex rings, interconnected by pairs of oppositely rotating line vortices, and shed at quasiregular intervals with a Strouhal number $\sim 0.19$. The rings are rapidly dissipated by turbulence diffusion, but it is shown that only the nascent vortex ring makes a significant contribution to the surface force, and that the force spectrum at Strouhal numbers exceeding unity is effectively independent of the shape of the fully formed vortex. Predictions of the lift and drag spectra at these frequencies are found to be in good accord with new towing tank measurements performed on a neutrally buoyant sphere configured as an acoustic velocity hydrophone. [Work supported by ONR Code 321 SS.]

\section{0:45}

4aEA11. Hydrodynamic lift and drag fluctuations of a sphereempirical model. J. Wang (The Trane Co., 3600 Pammel Creek Rd., LaCrosse, WI 54601-7599), G. C. Lauchle (Penn State Univ., State College, PA 16804), and M. S. Howe (Boston Univ., Boston, MA 02215)

An empirical model is developed to estimate the unsteady lift and drag induced on a spherical body subjected to a steady, uniform flow. A wind tunnel is used to measure the statistics of the surface pressure fluctuations on a sphere under subcritical Reynolds number conditions. The measurement results are incorporated into the model for predicting the unsteady force induced on the sphere. The model is based on a separable assumption of the cross-power spectral densities of the surface pressure fluctuations. This assumption is shown to be a proper engineering approximation except in low-frequency ranges. The model functions are obtained using least mean-square curve fits of the data. Tow tank experiments are performed, where the flow-induced unsteady side force and drag are measured independently of each other on spheres configured as acoustic velocity hydrophones. The empirical model and the tow tank measurements are in very good agreement with each other and also with a simplified vortex ring model presented in the accompanying paper. The predicted forces are used to determine the flow-induced noise on underwater inertial sensors subjected to a steady, uniform flow. [Work supported by ONR Code 321 SS.] 


\title{
Session 4aED
}

\section{Education in Acoustics and Noise: Public Education in Noise and Acoustics}

\author{
Daniel R. Raichel, Chair \\ 532 Spencer Drive, Wykoff, New Jersey 07481
}

\section{Invited Papers}

9:00

4aED1. Nonprofits and noise education, the experience of the Noise Pollution Clearinghouse. Leslie D. Blomberg (Noise Pollution Clearinghouse, Box 1137, Montpelier, VT 05601, les@nonoise.org)

During its four-year history, the Noise Pollution Clearinghouse has become a national leader in educating the public about noise and its effects. This paper explains the work of the Noise Pollution Clearinghouse, the reasons for its success, and the unmet needs and opportunities that remain. Particular attention is paid to the potential for nonprofits and professional societies to be leaders in noise education.

9:20

4aED2. Boom cars: Noise pollution at its worst. Daniel R. Raichel (Grad. School, CUNY, New York, NY 10031)

Boom cars, sometimes referred to as "boomers," are vehicles that have stereo sound equipment installed specifically to generate exceedingly loud sound levels. Because the market is so lucrative, particularly among automobile owners under 30 years of age, manufacturers aggressively engage in promotion of ultra-high-power amplifiers, auxiliary bass drivers, midrange drivers, tweeters, crossovers, etc. Loudness competitions are held; and levels as high as $170 \mathrm{~dB}$ have been reported. Most advertising emphasizes loudness - for example, a TV spot shows a van's sound system shattering store windows as it speeds down a street. In addition to an extremely high probability of hearing loss, those exposed to the excessive vibration inside a boomer's interior may experience other sorts of physiological trauma, this in addition to annoying the surrounding neighborhood with excruciatingly high sound levels. The phenomenon of boom cars compares to that of tobacco addiction- a danger to vehicle occupants and a nuisance to "secondary" listeners. Countermeasures against such public nuisances include: (1) enactment and diligent enforcement of municipal laws specifying limits on discernable sound levels, (2) education in secondary schools on the dangers of excessive noise levels, and (3) public criticism of manufacturers' promotional efforts that emphasize extremely high sound power.

\section{Contributed Papers}

9:40

4aED3. Noise control in buildings-A noise-control class for architectural engineers. Ralph T. Muehleisen (Dept. of Civil, Environ., and Architectural Eng., Univ. of Colorado, Boulder, CO 80309)

As public awareness of noise control grows and the public tolerance for noise diminishes, interest in building noise control is increasing. Since noise control is better applied as an integral part of the building design, rather than as an afterthought, building designers need to be educated in the basics of noise control. To this end a new class entitled Noise Control in Buildings has been developed for architectural engineering majors at the University of Colorado (CU). The class is a senior-level/graduate-level design class that introduces the basics of acoustics and noise control as applied to building design. In the class students learn from lecture, homework, guest lecturers from industry, field trips, experimental sessions, and projects. A discussion of the class content and teaching method is provided.

\section{9:55}

4aED4. The 1-day public symposium as a format for acoustics dissemination. Sten Ternstrom (Dept. of Speech, Music and Hearing, Kungl. Tekniska Hogskolan, Drottning Kristinas Vag 31, SE-100 44 Stockholm, Sweden, sten@ speech.kth.se)

The 1-day public seminar or symposium is a combined education and dissemination format that has been successfully applied by the Music Acoustics group at KTH in Stockholm, about once a year for the past 25 years. Such a seminar is typically structured as a full morning and afternoon of scheduled, invited lectures on a particular theme, followed by a public evening concert or other performance that corresponds to the theme. One or two international guests are keynote speakers, while the bulk of the program is contributed by leading Scandinavian researchers, teachers, and performers. The target style for the presentations is that of an introduction or overview for the interested and motivated but nonexpert academic. The topics are chosen for their expected public appeal, from ongoing or recent research activities in music acoustics and voice acoustics. Recent topics have included "Music and motion," "Real-time biofeedback in voice therapy and pedagogy," and "Randomness and noise in music." Attendance usually ranges from 70 to 200 . When possible the lectures are also published in book form, complete with sound examples. Aspects of content, public relations, and the financing of such symposia are discussed.

10:10

4aED5. "Sound" science and technical education for sixth graders: A practical approach. Harry Alter (19 Beechtree Ln., Granville, OH 43023) and Rebecca Evans (1655 River Rd., Granville, OH 43023)

Following the renovation of the Granville Middle School Cafeteria in Granville, $\mathrm{OH}$ it was noticed by students and faculty that noise levels during lunch period had dramatically increased. As a result, speech became unintelligible and eating became both unenjoyable and uncomfortable. The resident sixth-grade science teacher with the assistance of a local acoustical engineer saw the opportunity to teach the sixth-grade science class about acoustics and to get the children directly involved in solving the school's cafeteria noise problem. The class met on a weekly basis to 
learn about sound and its influences on the school environment. Ultimately, the students would build a single microphone impedance tube and test the sound absorption characteristics of various materials. From their investigation they would design and install an acoustical wall treatment system to reduce reverberant noise levels in the school's cafeteria. Acous- tical measurements of the cafeteria were taken before and after treatment to compare the student's calculations and final work. The paper also outlines the comprehensive effort involved from securing local grant monies to technical discussions and photographs of the children's work.

THURSDAY MORNING, 7 DECEMBER 2000

YAWL ROOM, 8:15 TO 9:45 A.M.

\title{
Session 4aMUa
}

\section{Musical Acoustics: Modal Analysis Techniques in Musical Acoustics}

\author{
Daniel A. Russell, Chair \\ Science and Mathematics Department, Kettering University, 1700 West Third Street, Flint, Michigan 48504-4898
}

Invited Papers

8:15

\begin{abstract}
4aMUa1. Application of modal analysis and synthesis of reed and pipe to numerical simulations of a clarinet. Matteo Facchinetti (Laboratoire d'Hydrodynamique, Ecole Polytechnique, 91128 Palaiseau Cedex, France, matteo@ladhyx.polytechnique.fr), Xavier Boutillon (Laboratoire d'Acoustique Musicale, CNRS, Universite Paris 6, Paris, France), and Andrei Constantinescu (Laboratoire de Mecanique des Solides, Ecole Polytechnique, 91128 Palaiseau Cedex, France)
\end{abstract}

A finite-element model of a complete clarinet is proposed in order to simulate the acoustical dynamics of the instrument. Following the work of Pinard and Laine on isolated reeds [unpublished reports of the Ecole Polytechnique], modal analysis of clarinet reeds performed with holographic interferometry has been compared with modal synthesis derived from the FEM. In this linear model, the reed is considered alone first, then coupled to the mouthpiece, and finally to the mouthpiece and the barrel. The good agreement between the two approaches makes the mixed solid-acoustical FEM usable in a numerical simulation of the whole clarinet. The rest of the pipe is modeled by lumped mechanical elements whose parameters are fitted with measurements of the acoustical input impedance. Eigenmodes of the complete system—reed, mouthpiece, barrel, pipe—are computed and damping is subsequently attributed to each mode. The evolution of the system subject to nonlinear boundary conditions-incoming airflow, one-sided contact of the reed against the curved lay of the mouthpiece-is computed in the time domain: at each time step, external forces and internal variables are projected onto the modal basis and the evolution of each modal amplitude is integrated. Findings of the simulations will be discussed.

8:45

4aMUa2. Modal analysis of musical instruments using TV holography. Thomas D. Rossing (Phys. Dept., Northern Illinois Univ., DeKalb, IL 60115)

Holographic interferometry gives the greatest spatial resolution of any method of modal analysis. However, recording holograms on photographic film is time consuming and often inconvenient. Electronic systems that use CCD TV cameras to record images and form the interferograms in a small digital computer avoid these inconveniences. In a typical electronic TV holography system, a new interferogram is displayed 30 times a second, providing the opportunity to view the interferograms in real time as some parameter is varied. We illustrate this method by describing the vibrational modes of several musical instruments, including bells, gongs, cymbals, drums, violins, cellos, guitars, mandolins, and steelpans.

\section{Contributed Papers}

9:15

4aMUa3. Recent work and new axes of research for MODALYS, a sound synthesis program based on modal representation. Cristophe Vergez, Joel Bensoam, Nicolas Misdariis, and Rene Causse (IRCAM, 1 Pl. Igor Stravinsky, 75004 Paris, France)

MODALYS, developed at Ircam after 10 years for research and musical applications, is based on modal representation of vibrating objects, mechanical or acoustical. Modal data (natural frequencies, damping factors, and deflection shapes) are obtained from: (1) analytic modeling for simple structures as ideal string, cylindrical or conical open/closed tubes, rectangular plate with a simply supported boundary condition or violin bridge,
...); (2) experimental measurements for real structures with complex shapes (violin body, soundboard of piano, flute, ...); and (3) finally by numerical methods (finite-element methods) which allow the creation of any arbitrary virtual shapes. The vibrating object involved is generally a set of simple and linear substructures. Nonlinearities, when they exist, are introduced in the coupling of substructures, for example reed and tube for the clarinet. Dissipation plays a critical role in the perception of some vibrating musical objects. The type of damping used in our modal representation is referred to as proportional damping (dissipation phenomena are in this case constant or proportional to the square of frequency). However, this type of damping may not apply in any case to the real objects. In this presentation, some sound illustrations on recent results of research and application development with MODALYs will be presented and discussed. 
4aMUa4. Acoustic and modal analysis of an African djembe drum. Daniel A. Russell and Wesley S. Haveman (Sci. \& Math Dept., Kettering Univ., 1700 W. Third Ave., Flint, MI 48504)

The African djembe drum consists of a goat skin stretched over a hand-carved shell with a large cavity, open at the bottom. The shape of the ponent around $75 \mathrm{~Hz}$. The shell exibits several bell- or wineglass modes, some of which have frequencies close to membrane modes. This talk will present results from acoustic and modal analysis tests of the drum with and without the skin membrane intact. Mode shapes and frequency spectra will be presented as well as a discussion of how the shell, cavity, and membrane couple together to provide the djembe's unique sound.

THURSDAY MORNING, 7 DECEMBER 2000

YAWL ROOM, 10:00 A.M. TO 12:00 NOON

\title{
Session 4aMUb
}

\section{Musical Acoustics: General Topics in Musical Acoustics}

\author{
Roger J. Hanson, Chair \\ Physics Department, University of Northern Iowa, Cedar Falls, Iowa 50614
}

Contributed Papers

10:00

4aMUb1. Bilingual speakers perceive a musical illusion in accordance with their first language. Diana Deutsch (Dept. of Psych., Univ. of California, San Diego, La Jolla, CA 92093), Trevor Henthorn (Univ. of California, San Diego, La Jolla, CA 92093), and Mark Dolson (E-mu Creative Technol. Ctr., Scotts Valley, CA 95067)

The tritone paradox is a musical illusion that is perceived quite differently by different listeners. Previous findings have indicated that perception of this illusion varies with the language or dialect to which the listener has been exposed, particularly in childhood. In this study, subjects were employed whose first language was Vietnamese and who now reside in California. One group of 16 subjects had arrived in the U.S. as adults. All spoke perfect Vietnamese but little English. Another group of 16 subjects had arrived in the U.S. as children. All spoke perfect English, but most did not speak fluent Vietnamese (though they still heard some Vietnamese spoken). The two groups perceived the tritone paradox in strikingly similar ways, and their perceptions differed significantly from those of another group of Californian subjects who were native speakers of American English. Another experiment, employing fluent Vietnamese speakers, obtained a close correspondence between the way a subject perceived the tritone paradox and the pitch range of his or her speaking voice. The bilingual subjects, therefore, perceived the tritone paradox in accordance with the pitch characteristics of their first language, even when they no longer spoke this language fluently.

\section{0:15}

4aMUb2. Laryngeal configuration and acoustical properties during flute vibrato. Joon-Hee Beth Hwang and C. William Thorpe (Faculty of Health Sci., The Univ. of Sydney, East St. Lidcombe, NSW 2141, Australia)

This study reports on simultaneous measurements of glottal aperture and spectral analysis of flute vibrato for a group of six expert flute players. The glottal aperture was recorded using a nasendoscope and the sound was recorded using a portable DAT recorder. Video images were selected from stable portions and digitized, and measurements of the vocal fold opening were made from the display. The sound recording was also digitized and was analyzed using MATLAB. The results showed an oscillatory pattern on the glottal opening together with the vibrato oscillations in the acoustic signal. The change in glottal opening between maximum and minimum adduction during this oscillation was an average of $7.42 \mathrm{~mm}$ for loud playing and $1.73 \mathrm{~mm}$ for soft playing. The spectral analysis also showed that the amplitude modulation occurs mostly in the higher partials of the tone. These results strongly suggest that the larynx plays an important role in developing vibrato in the flute tone. It appears that the larynx modulates the airflow, thus producing pressure fluctuations in the mouth that generate the oscillatory "vibration" of the acoustic tone as observed in previous studies [N. H. Fletcher, J. Acoust. Soc. Am. 57, 233-237 (1975)].

10:30

4aMUb3. A study of brass instrument "lipping" using an artificial mouth. Mark Neal, Francis H. Barnes, D. Murray Campbell, Clive A. Greated (Dept. of Phys. and Astron., Univ. of Edinburgh, Edinburgh EH9 3JZ, Scotland), John S. Cullen (Flat 12, Stow Pk. Ct., Newport NP20 4HB, UK), and Joel Gilbert (Universite du Maine, 72085 Le Mans Cedex 9, France)

Sound is generated in a brass wind instrument as a result of aeroelastic coupling between the mechanical vibratory system formed by the lips and the acoustical vibratory system of the air column. The sounding frequency depends on the parameters of both vibratory systems, and is in general not exactly equal to one of the acoustical resonance frequencies of the air column. A skilled brass instrument player can vary the method of blowing to pull the note above or below an acoustic resonance; this technique is known as "lipping." In order to obtain a clearer understanding of the lipping mechanism, an artificial mouth has been used to drive a trombone mouthpiece coupled to a cylindrical tube of variable length. The phase relationship between mouthpiece pressure and lip opening has been determined for near-threshold auto-oscillation over a range of air column lengths and embouchure adjustments, corresponding to lipping both above and below the acoustic resonance. Implications for the classification of the lip reed mechanism are discussed.

\section{0:45}

4aMUb4. Self-sustained oscillation as a function of blowing pressure in a one mass lip reed model. Jeffrey Moffitt and Daniel Ludwigsen (Dept. of Phys., The College of Wooster, 1189 Beall Ave., Wooster, OH 44691, jmoffitt@acs.wooster.edu)

The lip reed is one of the least understood components of the brass player-instrument system. Its biological nature allows a wide range of player control over the parameters that are vital to the regeneration of the pressure wave. The lumped-element model of Adachi and Sato [J. Acoust. Soc. Am. 99, 1200-1209 (1996)] accomplishes the goal of self-oscillation and displays the correct phase relationships with only two parameters to specify. The embouchure is captured by the natural frequency of the massspring system, and the blowing pressure must be sufficient to develop self-sustained oscillation. Now the behavior of a model player-instrument system can be compared with the results of the thorough investigation of blowing pressure of human players by Fletcher and Tarnopolsky [J. 
Acoust. Soc. Am. 105, 874-881 (1999)]. The 2D parameter space is mapped to show regions of self-sustained oscillation as a function of both blowing pressure and the lip's natural frequency. [Work supported by REU Grant NSF-DMR No. 9987850.]

\section{1:00}

4aMUb5. Effective lengths of trombone bells with mutes. Michael S. Lodin, R. Dean Ayers, and Lowell J. Eliason (Dept. of Phys. and Astron., California State Univ., Long Beach, 1250 Bellflower Blvd., Long Beach, CA 90840-3901, rdayers@csulb.edu)

A brass player employs mutes in performance to modify spectral characteristics of the sound output for musical effect. An undesirable side effect is a modification in pitch center, which is easily compensated for in the trombone by a small adjustment in playing slide position. The effects of various mutes upon the sounding frequencies of the trombone are studied by determining the change each mute produces in the effective length of an equivalent, closed-end air column. For both the open-bell case as well as with various mutes inserted, an impulse-response technique is used to determine the phase shift of each frequency component induced at the bell end, leading to a calculation of a frequency-dependent effective length for each configuration [R. W. Pyle, Jr., J. Acoust. Soc. Am. 57, 13091317 (1975); R. D. Ayers, ibid. 98, 81-87 (1995)]. The various lengths are compared with each other, as well as with the practical experience of brass players in using them during performance. [Work supported in part by the Scholarly and Creative Activities Committee at CSULB.]

\section{1:15}

4aMUb6. Nonlinear effects in an asymmetrical vibrating wire. Roger J. Hanson, H. Kent Macomber, and Andrew Morrison (Dept. of Phys., Univ. of Northern Iowa, Cedar Falls, IA 50614)

The asymmetries of a vibrating harpsichord wire which yield different free vibrational frequencies along two characteristic orthogonal transverse axes were described previously [Hanson et al., J. Acoust Soc. Am. 106, 2141 (1999)]. This splitting of the fundamental vibrational frequency is of the order of $0.3 \%$. Its value depends upon the particular wire sample and the tension, with lower tensions yielding larger absolute splittings. For the 2nd, 3rd and 4th vibrational modes, splittings differ greatly from integral multiples of the splitting of the fundamental. If the wire is driven sinusoidally there can be large nonlinear generation of motion in a direction $(P)$ perpendicular to the driving direction $(D)$ and of higher harmonics of the driving frequency. These nonlinear effects are, however, very sensitive to the frequency splitting and the relation of the driving direction to the characteristic axes. Theory indicates that $P / D \sim 1$, independent of the vibrational amplitude. However, with a small splitting of $0.11 \mathrm{~Hz}$ out of 77
$\mathrm{Hz}$, it was found that to obtain $P / D \sim 1$ an amplitude at the antinode of about $0.15 \%$ of the wire length was necessary. The driving frequency region for generation of higher harmonics is sharply dependent on the higher free-vibration mode frequencies.

\section{1:30}

4aMUb7. Study on double decay of individual partials of piano sound-Preliminary results on the "unison quality." Rene Causse (IRCAM, 1 Pl. Igor Stravinsky, 75004 Paris, France) and Simon Felix (Universite du Maine, 72017 Le Mans, France)

A characteristic of piano tones is their double decay (the initial sound followed by the "aftersound"). The difference in mistuning between the three strings of a unison group, and also the adjustment of the regularity of the action of the hammer in the case of important irregularities in hammer striking, are parameters used by the tuner to adjust the temporal evolution of the sound. This study is a part of a more general study to explore what tuners call "unison quality,', which means finding objective and qualitative criteria to decide that the temporal evolution is well adjusted. The results of experiments are reported where the decay of the first 12 partials of real sounds recorded under different conditions is analyzed and then modified by signal processing. The results of informal listening tests indicate that experts (tuner, professional pianists, and composers) are sensitive to these modifications (removal of very brief amplitude falls observed for the different partials). The decay of the different partials seems to be more complex than the double decay observed for the global sound and for the fundamental frequency. Sound illustrations will be presented.

\section{$11: 45$}

4aMUb8. Nonlinear modeling of the "mitnahme-effekt"' in coupled organ pipes. Domenico Stanzial, Davide Bonsi, and Diego Gonzalez (Lab. di Acustica Musicale e Architett. FSSG-CNR, Isola di S. Giorgio Maggiore, c/o Fond. Cini, I-30124 Venezia, Italia)

Two slightly mistuned organ pipes of the same nominal tone placed on neighboring holes of the same groove of the slider chest can synchronize if simultaneously sounded. The frequency difference between the two pipes disappears (mitnahme-effekt) giving up on a common frequency which is, in general, slightly higher than both natural frequencies of the pipes [Angster et al., "Coupling between simultaneously sounded organ pipes,' AES preprint 3534 (F1-2), Berlin (1993)]. A related phenomenon is observed when the separation between the two natural frequencies is bigger than $1 \%$ : this gives up on an increased difference frequency. This paper shows that a numerical simulation, where the two coupled pipes are modeled with a pair of coupled nonlinear oscillators, qualitatively obtains both these results. Furthermore the existence of a parameter range fitting all the experimental data is presented. 


\title{
Session 4aPA
}

\section{Physical Acoustics: Propagation and Mixed Physical Topics}

\author{
Roger M. Waxler, Chair \\ National Center for Physical Acoustics, University of Mississippi, Coliseum Drive, University, Mississippi 38677
}

\section{Contributed Papers}

8:00

4aPA1. A simple procedure for the selection of singular values in acoustical travel-time tomography. Amin Bassrei (Inst. of Phys. and CPGG/UFBA, Campus Univ. Ondina, 40210-340 Salvador BA, Brazil)

Inverse problems are in general ill-posed and acoustical travel-time tomography is no exception. One common way to solve this inverse problem is by the generalized inversion through singular value decomposition (SVD). The crucial issue that arises here is the avoidance of small singular values which generally contribute to inconsistent solutions. The selection of singular values used in this work is based on the analysis of some estimators: rms error between the true model and the estimated one, rms error between the observed data and the calculated one, rms of the model, and the entropy of the model. In a numerical simulation with $N$ equations and $N$ unknowns, the SVD technique provides $N$ singular values; $N$ inverse matrices are then built, using $1,2, \ldots$, up to all the $N$ singular values. For each matrix one solution is obtained and the above estimators are calculated and analyzed. Solutions with, for instance, small rms error are accepted, and solutions with inconsistent entropies are rejected. Thus, it is possible to determine the number of singular values related to the solution with a maximum of information and a minimum of discrepancy. [Work supported by CNPq and PGS.]

\section{8:15}

4aPA2. Greens function modified spherical harmonic analysis. John MacGillivray and Victor Sparrow (Grad. Prog. in Acoust., Penn State Univ., 217 Appl. Sci. Bldg., University Park, PA 16802, creeve@sabine.acs.psu.edu)

In an effort to develop a model for impulse responses in reverberant spaces, techniques for source radiation modeling were explored. One method of decomposing a source field involves an analysis based on spherical harmonic expansions of the field. Spherical harmonic decomposition is a powerful tool, but it is a mathematical approach which is completely independent of the type of source that creates the field. As a consequence, this technique cannot, in general, be used to gain insight into the underlying physical nature of the source. Moreover, because this is a brute force technique, the analysis can be extremely inefficient. In this presentation, modifications to the spherical harmonic analysis and synthesis equations are made to overcome these problems. These are based on the (theoretical) source types associated with the individual spherical harmonic expansion coefficients, and determination of the location of the primary components of the (physical) source. These modifications can greatly improve the computational efficiency and allow the source to be described by a set of coefficients which is more directly related to actual source types present. Illustrative examples will be shown. [Work supported by the Applied Research Laboratory at Penn State University under the Educational and Foundational Research Program.]
4aPA3. Infinite impulse response-based simulation of room acoustics. Parita Amin, Jing Tsui, and Charles Thompson (Ctr. for Adv. Computation and Telecommun., ECE, Univ. of Massachusetts, Lowell, Lowell, MA 01854, Charles_Thompson2@uml.edu)

In this work, the problem of filter construction for the point-to-point impulse response in a room is examined. Our particular interest is infinite impulse response (IIR) filters. It will be shown that such filters can be constructed from image-based models. The use of subband IIR filter models is investigated. Our model provides perfect reconstruction of early arrivals. The late arrivals in the impulse response are in agreement only in the least-squared sense. Experimental results will be given for the room impulse response.

\section{$8: 45$}

4aPA4. On the estimations of sound field by source-distributed methods. Gee-Pinn James Too (Dept. of Naval Architecture and Marine Eng., Natl. Cheng Kung Univ., Tainan, Taiwan 70101, ROC)

Source-distributed methods were developed earlier by Stepanishen et al. and many others to study the radiation sound field. There are several articles discussing the properties of these methods, although there are alternative names for the source-distributed methods. In the present study, the properties of these methods are summarized. The applications of source-distributed methods for radiation and scattering sound fields are demonstrated. It is interesting to show that the errors in the near-field measurement might be canceled out in the estimation in the far field. In the application for estimation of interior sound field, the source-distributed methods also give good results compared with the results of the Helmholtz integral formulation. In addition, it is pointed out that there are some restrictions in the methods. [Work supported by NSC of ROC.]

9:00

4aPA5. Application of random walk methods to wave propagation. Bair Budaev and David Bogy (Dept. of Mech. Eng., Univ. of California, Berkeley, \#1740, Berkeley, CA 94720-1740)

We present an approach to problems of diffraction that has its roots in a number of well-established theories such as the geometric theory of diffraction, the methods of Feynman's path integrals and Wiener's functional integrals, and the theory of stochastic processes. We start our analysis of the Helmholtz equation by closely following the scheme of the ray method, but instead of approximating the resulting second-order auxiliary equation by a first-order transport equation, we study the original auxiliary equation and obtain its exact solution as the mathematical expectation of a specified functional in the space of Brownian trajectories with the Wiener probabilistic measure. The obtained solution appears to be a direct improvement of the ray method approximation to the exact solution of the Helmholtz equation, and it is shown to admit efficient numerical evaluation. [Work supported by NSF Grant 9730779.] 


\section{9:15-9:30 Break}

9:30

4aPA6. On the square-root distance dependence of sound field near an edge of a semi-infinite plane. Mitsuhiro Ueda (Dept. of IDE, Tokyo Inst. of Technol., 2-12-1 O-okayama, Meguro-ku, Tokyo, 152-8552 Japan, ueda@ide.titech.ac.jp)

It is known that the 2-D sound field near an edge of a semi-infinite plane depends on the square-root of a distance between the observation point and the edge. The boundary value problem for the potential on the semi-infinite plane is formulated using a new principle of diffraction, that is, virtual discontinuity principle of diffraction (VDPD). According to this formulation, a new response function, that describes mutual dependence of potentials on the plane, is obtained and is localized near the edge. Since the square-root distance dependence also appears only near the edge, it becomes clear that the dependence can be explained by the analysis localized near the edge. Consequently the boundary value integral is carried out for a very short interval including the edge assuming that the observation point is very near the edge. The square-root dependence arises from the restriction that the same potential is reconstructed from the integration over the short interval. It is interesting to note that the new response function contains a scheme in it to fix the distance dependence of potential at points near the edge.

\section{9:45}

4aPA7. On the model of propagation of high-velocity subsurface waves. Edouard G. Nesvijski (Ctr. of Technol., Federal Univ. of Santa Maria, Campus Universitaio, Santa Maria, RS 97110-970, Brazil)

There are a great number of theoretical and experimental works published on the problem of analysis and classification of surface waves, but some recent experimental results cannot be explained unambiguously by existing basic theories because the velocity of registered waves is considerably higher than expected. For a better understanding of this phenomenon it is proposed to take a fresh look at the classical elaboration of the Rayleigh problem. This paper considers additional real roots of the Rayleigh equation in an attempt to explain the existence of a special type of high-velocity leaky waves propagating under the surface of half-space solid bordering with vacuum or gas. Their main energy concentrates in a local zone under the surface, and below this zone they transform into bulk waves taking away the wave energy. Some computer simulations and calculations are presented as a theoretical explanation of the conditions of high-velocity leaky subsurface waves propagation. An original representation of the Rayleigh function in 3-D gives one the opportunity to better understand the physical nature of the subsurface wave behavior in solids with different elastic characteristics. The proposed model of propagation of high velocity subsurface waves fits well with experimental data.

\section{0:00}

4aPA8. Stress measurement using critically refracted longitudinal waves. Don E. Bray (Don E. Bray, Inc., P.O. Box 10315, College Station, TX 77842)

Critically refracted longitudinal waves (LCR) offer considerable advantage in achieving nondestructive measurement of stress in engineering components and structures. Of the various wave types, the LCR is most sensitive to stress and least sensitive to texture. Also called the surface skimming longitudinal wave (SSLW), the LCR wave skims along just underneath the surface of a material at bulk wave speeds. Further, there is experimental evidence of frequency dependence where the higher frequencies concentrate their energy more toward the surface than do the lower frequency waves. This effect, which has not been fully explained theoretically, enables stress gradient evaluation below the surface. Further, there is observed scatter in the travel times found at low stress levels. At higher levels of stress, the data often become very linear. The specific nature of the LCR wave, acoustoelasticity, LCR probes and beam patterns, and methods for its excitation, are described. Probe frequencies from 1.0 to 20 $\mathrm{MHz}$ have been used. System travel-time repeatability is $0.01 \%$ or better and stress resolution of $\pm 21 \mathrm{MPa}(3 \mathrm{ksi})$ is obtained in typical applications. Tests showing the frequency dependence and the scatter observed at low levels will be discussed. Several applications described using the LCR technique for stress measurements include pressure vessels, piping, welds, titanium turbine engine blades, aluminum plate and a turbine rotor. Future applications and research using the technique will be mentioned.

\section{0:15}

4aPA9. Analysis of fields and forces on particles in an open-sided acoustic resonator using FEM with a perfect matched layer. Yibing Zheng and Robert E. Apfel (Dept. of Mech. Eng., Yale Univ., New Haven, CT 06520-8286, yibing.zheng@yale.edu)

The standing acoustic field in an axisymmetric open-sided resonator with a curved reflector is studied using the finite element method (FEM) with an anisotropic perfectly matched layer (PML) boundary condition. This kind of open-sided resonator is widely used in acoustic and acoustoelectric levitators, where the acoustic field is not considered as onedimensional due to the shape of the reflector. The method has been successfully employed to calculate the acoustic field profiles, the resonance frequencies, and the quality factors of the resonant modes. The numerical results of resonators with a concave or convex reflector are compared with those of the analytical asymptotic method. In the resonator of a real acoustic levitator, this method can calculate the acoustic fields driven by the surface vibration of the transducer, with and without particles levitated. The acoustic radiation forces on levitated particles are analyzed in such acoustic standing fields. The authors are trying to extend this study to characterize the levitation of a three-dimensional drop array in an acoustoelectric levitator. [Work supported by NASA (NAG3-2147) and by NSF (CTS-9870015).]

\section{$10: 30$}

4aPA10. The effect of time-dependent void fractions in the oscillations of wet foam drops. Iskander Akhatov (Inst. of Mech., Ufa Branch of the Russian Acad. of Sci., 6 Karl Marx St., Ufa 450000, Russia, iskander@anrb.ru), J. Gregory McDaniel, and R. Glynn Holt (Boston Univ., 110 Cummington St., Boston, MA 02215)

We develop a model for the nonlinear oscillations of spherical drops composed of aqueous foam. Beginning with a simple mixture law, and utilizing a mass-conserving bubble-in-cell scheme and a uniform pressure approximation, we obtain a Rayleigh-Plesset-type equation for the dynamics of bubbles in a foam mixture. The dispersion relation for sound waves in a bubbly liquid is then coupled with a normal-modes expansion to derive expressions for the frequencies of modal oscillations. These modal (breathing plus higher-order shape modes) frequencies are elicited as a function of the void fraction of the foam. A Mathieu-type equation is obtained for the dynamics of the higher-order shape modes and their parametric coupling to the breathing mode. Numerical results are presented. [Work supported by NASA.]

\section{0:45-11:00 Break}

\section{1:00}

4aPA11. A study of knock and adiabatic process in multi-fuel engines. Salvador Cerdá, José Romero, Joaquín Navasquillo (Lab of Acoust., Dept. of Appl. Phys., Univ. of Valencia, Spain), and Grover Zurita (Lulea Univ.)

For both engine designers and control purposes, a better understanding of the knocking that occurs during combustion in engines can lead to optimal decisions which will make engines operate more efficiently. The rapid rise in diesel engines is recognized as an audible impulse noise, which is known as "knock." The knock is caused by the spontaneous combustion of a significant volume of fuel/air mixture. The knock produces shock waves within the combustion chamber and results in a great number of combustion chamber resonance frequencies. The engine used in the experiments was a six-cylinder modified diesel engine running in ethanol conditions. The main purpose of this research is to determine the 
knock phenomena and the adiabatic process. To perform the data analysis a time-frequency spectral approach was used. The results revealed both radial and circumferential resonances. The most important adiabatic coefficients were carried out and used to calculate the heat release rate.

\section{1:15}

4aPA12. Sound attenuation in the critical region of the ionic binary mixture ethylammonium nitrate/ $\boldsymbol{n}$-octanol. Sirojiddin Z. Mirzaev (Heat Phys. Dept., Uzbek Acad. of Sci., Katartal 28, Tashkent 700135, Uzbekistan) and Udo Kaatze (Drittes Physikalisches Institut, Georg-August-Universitaet, 37073 Goettingen, Germany)

Ultrasonic attenuation spectra $(100 \mathrm{kHz}<f<4 \mathrm{MHz})$ of the ionic mixture ethylammonium nitrate $/ n$-octanol of critical composition and also of the ethylammonium nitrate itself $(100 \mathrm{kHz}<f<400 \mathrm{MHz})$ are discussed at different temperatures. The measured spectra are evaluated in terms of the Bhattacharjee-Ferrell dynamic scaling theory. Using literature data for the amplitude of the fluctuation correlation length, the background and critical part of the heat capacity, the sonic attenuation spectrum as predicted by the Bhattacharjee-Ferrell model has been calculated for the critical mixture at the critical temperature. Again following this theoretical model, the contribution due to concentration fluctuations has been subtracted from the measured spectra at the temperatures of measurement and also subtracted has been the high-frequency asymptotic background contribution. The measured data are consistent with the Bhattacharjee-Ferrell theory of critical concentration fluctuations but show an additional frequency dependence. [Work supported by the Volkswagen-Stiftung, Hannover, Germany.]

\section{$11: 30$}

4aPA13. Results of attenuation calculations for a three-component gas mixture. Yefim Dain and Richard M. Lueptow (Dept. of Mech. Eng., Northwestern Univ., 2145 Sheridan Rd., Evanston, IL 60208, r-lueptow@northwestern.edu)

Acoustic attenuation in a mixture of gases results from the combined effects of molecular relaxation and the classical mechanisms of viscosity and heat conduction. As a result, the attenuation depends on the composition of the gas, frequency, temperature, and pressure. A model of the relaxational attenuation that permits the prediction of acoustic attenuation is used to predict the effect of composition, frequency, temperature, and pressure on the acoustic attenuation in a three-component gas mixture of nitrogen, methane, and water vapor. The attenuation spectrum is dependent upon the composition through the appearance of peaks in the spectrum related to the relaxation frequency of the particular components and their relaxing complexes. The relaxation peak related to methane dominates the spectrum except at low methane concentrations, where the nitrogen peak, which is dependent upon the water vapor concentration, is evident. Temperature and pressure significantly alter the relaxation frequency and the degree of attenuation, but water vapor plays little role in the attenuation at moderate and high methane concentrations. [This work was funded by the Department of Energy under subcontract from Commercial Electronics, Inc. (Broken Arrow, OK) and by the Ford Motor Company.]

\section{1:45}

4aPA14. A study of simulation methods of limited diffraction beams. Hu Peng, Zuosheng Zhang (Univ. of Sci. Tech. China, Hefei, PROC 230026), and Jian-yu Lu (Univ. of Toledo, Toledo, OH 43606)

Limited diffraction beams such as Bessel beams and $X$ waves have been studied extensively because of their potential applications in many areas such as medical imaging and optical communications. Computer simulations have been powerful tools for understanding the fundamentals of these beams. In this study, limited diffraction beams, specifically, Bessel beams and $X$ waves, were simulated. The simulations were based on the frequency-domain method (Rayleigh-Sommerfeld diffraction formula), time-domain method, and finite-element or finite-difference method. For Bessel beams, a circular transducer divided into multiple rings was assumed. For pressure distributions on axial axis, the simulations were simplified due to the symmetry. In the case of $X$ waves, the differential property of acoustic waves was used. The results of the simulation methods were compared with each other. Advantages and disadvantages of these methods will be reported. In all cases, the results are very close to those predicted by theory. [This work was supported in part by Grant HL 60301 from the National Institute of Health of the U.S.A.]

\title{
Session 4aPP
}

\section{Psychological and Physiological Acoustics: Pitch, Loudness and Localization}

\author{
Kourosh Saberi, Chair \\ Department of Cognitive Sciences, University of California, Irvine, California 92697
}

\section{Contributed Papers}

8:30

4aPP1. Franssen à la phones. William M. Hartmann, Scott R. Lawton (Phys. and Astron., Michigan State Univ., East Lansing, MI 48824), and Brad Rakerd (Michigan State Univ.)

According to current explanation, the Franssen effect reflects a competition between localization cues from the onset of a tone and cues from the steady-state portion. For a sine tone in a room, the steady-state interaural time and level cues (ITD and ILD) are implausible because of room reflections. Therefore, onset cues dominate, and a listener localizes the tone at its onset position regardless of the true origin of the steady state.
To test this explanation we used headphones to simulate the simplest kind of Franssen stimulus: The ITD was fixed throughout the tone. At onset, the ILD was zero. After onset, the ILD grew so as to contradict the ITD in the steady state. Equal numbers of trials were done with abrupt and slow onsets. The listener's task was to localize the steady-state part of the tone. A Franssen analog was identified by comparing responses to abrupt-onset and slow-onset tones. Results were compared with the standard Franssen effect in a large room. Clear evidence of a Franssen effect, with its dependence on plausibility, appeared in the headphones experiments, but the data were less dramatic than data obtained in the room. [Work supported by the NIDCD.] 
five observers. For every participant, psychometric functions were assessed at five hearing levels ranging from 40- to $80-\mathrm{dB}$ HL. The outcome

4aPP2. Specific learning phenomena in pitch discrimination tasks. Laurent Demany, Catherine Semal, Cecilia Maubaret, and Odon Noblia (Lab. de Neurophysiol., CNRS and Univ. Victor Segalen, 146 rue Leo Saignat, F-33076 Bordeaux, France, Laurent.Demany@psyac.u-bordeaux2.fr)

In experiment 1 , listeners were trained to discriminate, during ten sessions (11000 trials), a monaural pure tone of $3000 \mathrm{~Hz}$ from ipsilateral pure tones with slightly different frequencies. The resulting perceptual learning (improvement in thresholds) appeared to be frequency-specific since, in subsequent sessions, a new learning was observed when the tone frequencies were shifted to a range near $1200 \mathrm{~Hz}$, or $6500 \mathrm{~Hz}$. By contrast, when the initial tones were subsequently presented to the contralateral ear, thresholds were immediately optimal; therefore, the initial learning was not ear-specific. In experiment 2, performed on different listeners, training in pitch discrimination was initially provided using complex tones that consisted of harmonics 3-7 of a missing fundamental (near $100 \mathrm{~Hz}$ for some listeners, $500 \mathrm{~Hz}$ for others). After 11000 training trials, these complex tone stimuli were replaced by pure tones with frequencies that compared to the complex tone frequencies in three different ways: (condition A) their missing fundamentals, (condition B) their median spectral components, (condition C) completely novel. New learning was observed in each condition. Although the pure tones used in condition A were matched in pitch to the previously presented complexes, there was no evidence for a transfer of learning in this condition.

9:00

4aPP3. The upper boundary of temporal pitch coding inferred from electric stimulation of the auditory nerve. Fan-Gang Zeng (Univ. of California, 364 Med Surge II, Irvine, CA 92697)

Pitch can be represented by a vibration pattern in the cochlear partition (the place code) or a spike firing pattern in the auditory nerve (the temporal code). Generally, pitch of high tones has been assumed to use the place code and pitch of low tones to use the temporal code. Because both place and temporal codes change simultaneously as a function of frequency in a normal auditory system, the upper boundary of temporal encoding of pitch is not clear. Here, we use cochlear implants to infer the upper boundary of temporal pitch coding. The cochlear implant electrically stimulates the auditory nerve, providing an opportunity to assess the temporal pitch coding independent of the place code. Cochlear implant listeners performed pitch estimate and discrimination tasks as a function of frequency. The tasks were performed when either basal or apical electrodes were stimulated independently. The results showed that pitch is monotonically increased for frequencies lower than $300 \mathrm{~Hz}$ and does not change for frequencies above that. Pitch discrimination results also showed a break point at about $300 \mathrm{~Hz}$. Together, the present results suggest that $300 \mathrm{~Hz}$ is the upper boundary of temporal pitch coding.

\section{9:15}

4aPP4. Testing a new theory of Fechnerian scaling: The case of auditory intensity discrimination. Karin Zimmer and Hans Colonius (Inst. fur Kognitionsforschung, FB5/A6, Universitat Oldenburg, 26111 Oldenburg, Germany)

Recently, Dzhafarov and Colonius [Psych. Bull. Rev. 6, 239-268 (1999)] proposed a theory that refines Fechner's ideas of deriving the perceptual distance between stimuli from discriminability measures in the unidimensional case, and generalizes it to multidimensional stimulus domains. In this theory, the existence of a Fechnerian metric is based on testable assumptions about the shape of the psychometric functions. Thus, "same-different" psychometric functions: (1) have to attain a single minimum at some point; (2) must be locally symmetric around their minima; and (3) must show the same degree of cuspidness, or flatness, along the stimulus continuum. In the present investigation, these assumptions were evaluated experimentally employing acoustical stimuli differing along one stimulus dimension, intensity. In a "same-different" paradigm, using the method of constant stimuli, seven $1000-\mathrm{Hz}$ tones deviating from 0 to $2.1 \mathrm{~dB}$ with respect to a standard were presented diotically to of the "same-different" experiments generally supports the assumptions of the theory. Therefore, data such as these are, in principle, suitable for deriving a global loudness scale from local discriminability measures.

\section{9:30}

4aPP5. Intensity-difference limens as a function of level for Gaussianshaped packets of broadband noise. Lance Nizami, Walt Jesteadt, and Jason F. Reimer (Boys Town Natl. Res. Hospital, 555 N. 30th St., Omaha, NE 68131, nizami@boystown.org)

The nonmonotonic level dependence of the intensity-difference limens (DLs) for a broadband stimulus, the click [Raab and Taub, J. Acoust. Soc. Am. 46, 965-968 (1969)], can also be observed for a narrow-band stimulus, a Gaussian-enveloped 2-kHz tone [Nizami et al., J. Acoust. Soc. Am. 107, 2820 (2000)]. Although the DLs for a 32-ms tone-pip (measured at plus-or-minus four standard deviations) follow the near-miss to Webers Law, the DLs for a 4-ms tone-pip are higher, and peak mid-level, at about $40 \mathrm{~dB}$ SL. The DLs are larger still, and the peak more profound, for an 8-ms tone-pip. Energy-spectrum changes as duration changes, however, obscuring the source of the nonmonotonicity. Energy-spectrum was therefore held constant by using a broadband-noise carrier, while preserving the Gaussian envelope. DLs were obtained from two male and four female subjects (ages 18-40) having normal hearing thresholds. Noise-pip levels ranged from 30 to $90 \mathrm{~dB}$ SPL at durations of 2, 4, 8, 16, and $32 \mathrm{~ms}$. Although the across-subject mean DLs for 32- and 16-ms noise-pips follow Weber's law, the means for $8 \mathrm{~ms}$ are larger, and rise at mid-level. The DLs grow as duration decreases from 8 to 4 to $2 \mathrm{~ms}$, unlike the case for 2 $\mathrm{kHz}$.

\section{$9: 45$}

4aPP6. Temporal weights for loudness comparisons. Wolfgang Ellermeier and Sabine Schrödl (Institut für Psychologie, Universität Regensburg, 93040 Regensburg, Germany)

Temporal integration of the loudness of level-fluctuating sounds was investigated using the trial-by-trial analysis developed by Berg [J. Acoust. Soc. Am. 86, 1743-1746 (1989)]. In a two-interval, forced-choice procedure, two noise samples of 1-s duration were presented on each trial. Their levels were randomly varied every $100 \mathrm{~ms}$ by repeatedly, and independently, drawing from normal distributions having expected values of 60 or $61 \mathrm{~dB}$ SPL, respectively. The subject's task was to decide which of the two noise samples sounded louder. Based on a few thousand judgments, conditional psychometric (COSS) functions were estimated in order to determine to what extent each temporal segment contributed to the judgment. Data collected on six subjects showed considerable individual differences in the weighting functions, but-in contrast to averaging techniques widely used in sound evaluation-exhibited a statistically significant trend to accentuate the onset of the noise. These results were compared to the outcome of a second experiment, in which the total duration of the noise samples was shortened to $200 \mathrm{~ms}$ to address a different stage of echoic memory. Conclusions regarding the sensory and cognitive mechanisms involved in short-term loudness integration will be presented.

\section{0:00-10:15 Break}

\section{0:15}

4aPP7. Effect of frequency region and noise bandwidth on the AMFR. Shaum P. Bhagat and Craig A. Champlin (Univ. of Texas, Austin, Austin, TX 78712)

This study investigated the effect of varying stimulus frequency region and noise bandwidth on the amplitude modulation following response (AMFR). The stimulus for the AMFR was an amplitude-modulated burst of bandlimited white noise. Two carrier frequency regions (1 and $4 \mathrm{kHz})$ and two noise bandwidths $(100 \mathrm{~Hz}$ and $1 \mathrm{kHz})$ were employed. Three women and three men with normal hearing participated in the study. Low carrier-frequency regions produced significantly larger AMFRs than high 
carrier-frequency regions. Wide-noise bandwidths generated significantly larger AMFRs than narrow-noise bandwidths. The noise bandwidth effect was dependent on the rate of amplitude modulation. Modulation transfer functions were derived by plotting the AMFR amplitude as a function of modulation rate. At the greatest modulation depths, the transfer function resembled a bandpass filter. For lower modulation depths, the transfer function resembled a low-pass filter. The results of the study are in general agreement with previous research that investigated the AMFR with tone and wideband noise carrier signals.

\section{0:30}

4aPP8. Distance localization using nonindividualized head-related transfer functions. Pavel Zahorik (Dept. of Psych. and Waisman Ctr., Univ. of Wisconsin-Madison, Madison, WI 53705)

Past research has suggested that virtual auditory displays are most accurate when implemented using individualized head-related transfer functions (HRTFs). Directional localization accuracy, for example, is often substantially degraded when nonindividualized HRTFs are used, due to the resulting distortion of spectral cues to sound source direction. Although equally important for accurate spatial representation, little is known about distance localization accuracy in virtual auditory displays, and specifically, whether individualized HRTFs are necessary for accurate distance localization. Here, distance localization accuracy was assessed for six listeners, using both individualized and nonindividualized HRTFs in separate blocks of trials. The virtual sound sources were produced from special HRTF measurements (source distance ranging from 0.3 to $13.8 \mathrm{~m}$ ) that also contained the acoustic transfer characteristics of a semireverberant room environment. This provided listeners with additional acoustic cues to source distance, including the ratio of direct-to-reverberant sound energy. Since no significant differences were observed in listener's judgments of apparent sound source distance in the two stimulus conditions, it is concluded that distance localization accuracy, and degree of sound source externalization, do not depend on the use of individualized HRTFs when natural reverberation is available to the listener. [Work supported by NIH-NIDCD, NASA, and NIH-NEI.]

\section{$10: 45$}

4aPP9. Reverberant speech intelligibility for monaural and binaural listening. Brad Libbey and Peter Rogers (Mech. Eng., Georgia Inst. of Technol., Atlanta, GA 30332-0405, gt1556a@prism.gatech.edu)

Humans listening to speech in a small room are frequently unaware of reverberation. It is unknown if neurological processes remove these echoes or if they are simply disregarded when speech is phonetically processed. In other words, is there a neurological mechanism that is capable of removing echoes to create a clean speech neurological signal before phonetic processing? Or is the brain capable of processing reverberant phonemes? These questions have been explored in the process of investigating the hypothesis; "Binaural listening does not improve speech intelligibility.' Previous experiments have shown that binaural listening does not improve intelligibility for highly reverberant small rooms, T60 $=1.5 \mathrm{~s}$. The current experiment investigates the intelligibility of words in three reverberant rooms, $\mathrm{T} 60=1.1,0.7$, and $0.4 \mathrm{~s}$. Subjects listen with one or two ears to lists of words taken from a standardized testing procedure, ASA 85. In addition the rooms are simulated using the method of images and the reverberant words are presented in a diotic and binaural format over headphones. Intelligibility results from these rooms with monaural and binaural listening are presented.

\section{1:00}

4aPP10. A model to simulate human sound localization and detection in presence of a distracter. Jonas Braasch (Institut für Kommunikationsakustik, Ruhr-Universität, Bochum, Germany)

To simulate human auditory localization performance of a target in the presence of a distracter, existing models focus the analysis of the targets on frequency bands or time gaps where the distracter is not present. However, human localization also works quite well if the frequency ranges of target and distracter are identical and the distracter is played during the complete duration of the target-as long as the onsets of target and distracter are separated in time. This is taken into account in the proposed model. For each frequency band, the interaural time differences of the target are calculated from the difference of the interaural cross correlation (ICC) of the total sound (target+distracter) and the ICC of the distracter alone $\left(\Phi_{T}=3 D \Phi_{T D}-\Phi_{D}\right.$, assuming that target and distracter are uncorrelated). The latter is calculated from the part of the distracter that precedes the target. A similar procedure is used to measure the interaural level differences of the target. Auditory events are simulated assuming an ideal observer and internal noise. The model is able to mimic a number of psychoacoustic effects, including spatial shifts of the auditory events for low SNR situations. [Work supported by DFG.]

\section{1:15}

4aPP11. Individualized HRTFs using computer vision and computational acoustics. Ramani Duraiswami, Larry Davis, Shihab A. Shamma, Howard C. Elman (Inst. for Adv. Computer Studies, Univ. of Maryland, College Park, MD 20742), Richard O. Duda, V. Ralph Algazi (Univ. of California, Davis, Davis, CA 95616), Qing-Ho Liu (Duke Univ., Durham, NC 27708), and S. T. Raveendra (Automated Anal. Corp., Ann Arbor, MI 48104)

For a given sound source location and frequency content, the sound received at the two ears of a person is first scattered by his/her torso, head, and pinnae, leading to interaural differences in the intensity and spectral features of the sound received. These features are used as cues to perceive the spatial location of the source, and are encoded in an extremely individual "head-related transfer function" (HRTF) that depends on the person's external anatomy (structure of the torso, head, and pinnae). This individuality has made it difficult to use the HRTF to render perceptually convincing spatially distributed audio for applications such as entertainment or virtual reality. We have commenced a long-term project that will use numerical methods to compute individualized HRTFs from accurate 3D surface models of a person's torso, head, and pinnae (obtained using computer vision techniques). These surface models will be used with boundary-element methods to solve the wave equation and compute the HRTFs. An overview of our long-term project, as well as preliminary results that compare the numerical techniques to be used with analytical and experimental results for scattering from simple shapes, will be presented. [Work supported by NSF.]

\section{$11: 30$}

4aPP12. AM depth versus degree of amplitude fluctuation: Implementation error, adjustment, and implications. Pantelis Vassilakis (Systematic Musicology, Music Percept. and Acoust. Lab., UCLA, Box 951657, Los Angeles, CA 90095-1657)

AM depth values are often used in signal processing as a measure of amplitude fluctuation. In such cases, AM implementation produces an error reflecting the nonlinear relationship between the presumed and applied AM depths. A distinction has been made between two concepts of AM depth: (a) "spectral AM-depth," referring to the relationship between AM depth and degree of spectral energy spread and (b) "envelope AMdepth," referring to the relationship between AM depth and degree of amplitude fluctuation. The usual AM implementation equation represents consistently only the "spectral AM-depth." It is shown that, in order to apply an intended "envelope AM-depth," an adjusted AM depth coefficient must be inserted in this equation. The adjustment affects studies where changes in the degree of amplitude fluctuation have been correlated to changes in some physical, physiological or perceptual measurement. In general, all functions derived from the results of studies based on the miscalculation of amplitude fluctuation have been distorted by a factor proportional to the identified error. If and how this distortion influenced the interpretation of the provided functions will vary according to the importance and meaning attributed to each specific functions shape. Specific examples and general implications are discussed. 
11:45

4aPP13. Audiometric tests with various earphones, signals of short duration, and signals of high frequencies. Morten Lydolf, Brian L. Karlsen, Arturo O. Santilln, and Henrik Moller (Dept. of Acoust., Aalborg Univ., Fredrik Bajers Vej 7B-4, DK-9220 Aalborg O, Denmark, acoustics@acoustics.dk)

New audiometric standards on tests with signals of short duration and tests with signals of high frequencies are in the course of preparation by the international working group, ISO/TC 43/WG 1 . In this paper, first a selection of five different audiometric earphones is compared by using threshold measurements in the frequency range $125 \mathrm{~Hz}-8 \mathrm{kHz}$. These data are meant for validation of the reference threshold for regular pure-tone audiometry given in ISO 389-1. Signals of short duration consist of either rectangular pulses with a duration of $0.1 \mathrm{~ms}$ or pure-tone samples including only seven full periods at the octave band frequencies $0.5-8 \mathrm{kHz}$. Both single events and bursts with 20 signals presented during $1 \mathrm{~s}$ were used as stimuli. Finally, audiometric tests were made with high-frequency pure-tone stimuli of 1-s duration at: 8, 9, 10, 11.2, 12.5, 14, and $16 \mathrm{kHz}$. For all experiments the chosen psychophysical method was the ascending method, implemented nearly as described in ISO 8253-1. Twenty-five young, otologically normal test subjects were used in order to contribute to the standardization work. Representative reference thresholds are needed with respect to signals of short duration for the coming ISO 389-6 and for high frequencies in ISO 389-5.

\title{
Session 4aSA
}

\section{Structural Acoustics and Vibration: Acoustic Microsensors}

\author{
Vasundra V. Varadan, Chair \\ 212 Earth Engineering Sciences Building, Pennsylvania State University, University Park, Pennsylvania 16802 \\ Chair's Introduction-8:25 \\ Invited Papers
}

8:30

4aSA1. Acoustic atomic force microscopy applications in nanotechnology. Bernhard R. Tittmann, Weilou Gao, and Jikai Du (Dept. of Eng. Sci. and Mech., Penn State Univ., University Park, PA 16802)

In this work, acoustic atomic force microscopy is presented for nanometer material characterization. A transducer with a frequency range from $0.1 \mathrm{kHz}$ to $1 \mathrm{MHz}$ is typically fixed under a sample. The resonant frequency for the composite system is determined experimentally. The amplitude and phase images of the vibrating composite are obtained simultaneously as well as surface topography. Plasm spray drying process is studied by use of the acoustic AFM. Enhanced contrast in amplitude images shows unique capability to determine the plasm distribution in Alumina matrix. Further study is conducted on the contrast mechanism with a sample comprised of three different materials: $\mathrm{Si} 3 \mathrm{~N} 4, \mathrm{Cu}$, and steel. Theoretical analysis based on finite-element methods (FEM) and Johnson, Kendall, Roberts (JKR) model is conducted to simulate the system and determine the resonant frequency, amplitude, and phase. The results of the numerical simulations compare favorably with the experiment results. Furthermore, an amplitude-based method is proposed for estimating the material modulus through a semitheoretical, seminumerical process. The method shows higher sensitivity than the resonant frequency-based method. Also, microelectronic devices are studied for the analysis of diffusion.

\section{9:00}

4aSA2. Silicon micromachined ultrasonic transducers for bulk, Lamb, and Rayleigh waves. B. T. Khuri-Yakub (E. L. Ginzton Lab., Rm. 11, Stanford Univ., Stanford, CA 94305-4085, khuri-yakub@stanford.edu)

Capacitive micromachined ultrasonic transducers (cMUT) can match and even outperform piezoelectric transducers in terms of efficiency and bandwidth. With the advent of silicon micromachining, it is now possible to make capacitors with very thin gaps that sustain electric fields of the order of $109 \mathrm{~V} / \mathrm{m}$ or more. At these levels of electric field, the transformer coupling between the electrical and mechanical parts of the capacitor transducer, and thus its performance, are comparable to that of piezoelectric transducers. We will review the design and performance of cMUTs for airborne, immersion, Lamb and Rayleigh waves' ultrasound applications. We will show examples of immersion transducers (single element, 1-D and 2-D arrays of elements), air transducers and Lamb wave transducers. We will also present transducer systems (single element, 1-D and 2-D arrays of elements) with dynamic ranges of over $150 \mathrm{~dB} / \mathrm{Volt} / \mathrm{vHz}$, which are in excellent agreement with the theoretical predictions.

9:30

4aSA3. Wireless surface acoustic wave and MEMS-based microsensors. Vijay K. Varadan (Penn State Univ., 212 Earth/Eng. Sci. Bldg., University Park, PA 16802)

The integration of surface acoustic waves (SAW), microelectromechanical systems (MEMS), and required microelectronics and conformal antennas to realize programmable microsensors suitable for many engineering and biomedical applications will be reviewed in this talk. This unique combination of technologies results in novel conformal sensors that can be remotely sensed by a wireless communication system with the advantage of no power requirements at the sensor site (passive sensor). The required features in many 
of these applications are high precision, wide dynamic range, and wide frequency range. The MEMS-SAW-based devices presented possess typical advantages of MEMS sensors including the additional benefits of robustness, excellent sensitivity, surface conformability, and durability. After a brief overview of SAW sensors and MEMS, the paper is focused on the design and fabrication of MEMS devices for engineering and medical applications, for example: accelerometer and gyroscopes for automobile, inertial navigation; sensors for pressure, torque, temperature, and strain; health monitoring of structures; "smart tongue and electronic nose;" micropump for drug delivery; humidity, temperature, and ethlyne oxide sensor for hospital sterilization system; microsensors for monitoring bone healing; and pollution and chemical sensing.

10:00-10:15 Break

10:15

4aSA4. Finite-element method for determination of electromechanical coupling coefficient for piezoelectric and capacitive micromachined ultrasonic transducers. John D. Fraser (ATL Ultrasound, a Philips Medical Systems Co., 22100 Bothell Everett Hwy., P.O. Box 3003, Bothell, WA 98041-3003, john.fraser@philips.com) and Paul Reynolds (Weidlinger Assoc., Inc., Los Altos, CA 94022-1049)

Research has been reported on ultrasonic transduction using capacitive micromachined ultrasonic transducers (cMUTs). These are thin membranes of diameters of order $100 \mu \mathrm{m}$ suspended over a silicon substrate with a gap of order $1 \mu \mathrm{m}$. The devices become transducers when a bias voltage is applied, causing electrostatic forces to draw the membranes closer to the substrate. Operation is by applying an electrical signal to excite a vibration in the membrane, or an ultrasonic wave to excite an ac voltage. The cMUT literature includes equivalent circuits and studies on losses and spurious waves, and discussion of output, sensitivity, and bandwidth. Transducer engineers would like to see analogies to the properties of piezoelectric transducers, in order to compare the cMUTs to traditional designs. An extension of the definition of the piezoelectric coupling coefficient to an effective coupling coefficient for cMUT transducers is presented. A finite-element method has been developed using the PZFLEX software package to determine its value, and used for evaluating cMUT designs. Accepted values are obtained for a piezoelectric material, and it is shown that an ideal cMUT has a coupling coefficient approaching 1 at the membrane collapse voltage, while practical devices are often limited to lower values.

10:45

4aSA5. Computational modeling and analysis of SAW devices. Guanshui Xu (Dept. of Mech. Eng., Univ. of California at Riverside, Riverside, CA 92521)

Direct full-scale computational simulation of electronic acoustic wave devices is among the most challenging problems for designing high performance devices. The problem also becomes urgent as the conventional methods fail to characterize the behavior of miniaturized devices that operate at high frequency for demanding applications. The effective computational methodology has not yet been developed for the full-scale simulation of multi-physical phenomena in many micro-electromechanical devices of complex geometric structure on the nano- and micro-scale. Here a direct finite element model for the full-scale analysis of the frequency response of surface acoustic wave (SAW) devices is presented. The ability of the method to quantitatively evaluate the influence of the bulk waves at the high-frequency end of the filter passband and the influence of the number of electrodes on insertion loss is noteworthy. Some practical computational challenges of finite element modeling of SAW devices are also discussed parenthetically.

\section{Contributed Papers}

\section{1:15}

4aSA6. Development of a $\mathrm{SAW}$ microgas sensor for monitoring $\mathrm{SO}_{2}$ gas. Yongrae Roh, Youngjin Lee, and Hakbong Kim (Dept. of Sensor Eng., Kyungpook Natl. Univ., Sankyug-Dong 1370, Taegu 702-701, Korea, yryong@eeg.kyungpook.ac.kr)

We have developed a new type $\mathrm{SO}_{2}$ gas sensor applying a particular inorganic thin film on SAW devices. The sensor consists of twin SAW oscillators of the center frequency of $54 \mathrm{MHz}$ fabricated on $\mathrm{LiTaO}_{3}$ piezoelectric single crystals. One delay line of the sensor is coated with a $\mathrm{CdS}$ thin film that selectively adsorbs and desorbs $\mathrm{SO}_{2}$, while the other is uncoated for use as a stable reference. Deposition of CdS thin film has been carried out by the spray pyrolysis method using an ultrasonic nozzle. Mass loading induced by the $\mathrm{SO}_{2}$ gas adsorbed onto the CdS film results in corresponding frequency shifts directly proportional to the gas concentration. The relative change in the frequency of the two oscillators is monitored with a digital signal processing circuit. $\mathrm{SO}_{2}$ sensor properties investigated include selectivity, sensitivity, response time, and repeatability. The sensor shows promising performance as a microsensing tool and is capable of measuring concentrations in air less than 200 parts per billion of $\mathrm{SO}_{2}$.
11:30

4aSA7. Wireless surface acoustic wave ice sensor. K. A. Jose, S. Gangadharan, V. V. Varadan, and V. K. Varadan (212 Earth-Eng. Sci. Bldg., Penn State Univ., University Park, PA 16801)

Ice formation on an aircraft can cause serious safety problems, and hence, there is a real need for ice detection and monitoring on aircrafts. It is well known that acoustic wave devices offer a simple and inexpensive technique for a number of sensing applications. In this paper, we present a wireless ice detection and monitoring system using a surface acoustic wave (SAW) device utilizing shear horizontal (SH) waves as the sensing mechanism. The SAW device is designed and fabricated on a quartz substrate. Silicon dioxide is then deposited to serve as a guiding layer for $\mathrm{SH}$ waves. The acoustic wave concentrated in the guiding layer is highly sensitive to surface perturbations. The experimental setup used to measure the effect of ice formation is by an oscillator setup. The SAW device is designed to oscillate at a particular frequency and any fractional change in wave velocity due to surface perturbations leads to a change in oscillation frequency. It is observed that phase transformation from water to ice causes a shift in oscillator frequency of $2 \mathrm{MHz}$. The ice sensor can be operated wirelessly by connecting the SAW sensor to a stand-alone oscillator and the oscillator can communicate with a remote monitoring system using a microstrip antenna. 
4aSA8. Solid-state MOSFET-based micro hydrophone array. Bei Zhu, J. Ziang, Vijay K. Varadan, and Vasundara V. Varadan (Penn State Univ., University Park, PA 16802)

The fabrication of low-cost, high-sensitivity, and miniaturized hydrophone array is highly desirable for application in sonar technology. However, as hydrophone size decreases, it becomes necessary to provide an amplifier or buffer in close proximity to avoid sensitivity loss due to interconnect capacitance. This suggests the concept of hydrpophone integration with microelectronics in an integrated circuit environment. A proptotype of an integrated transducer was thus realized in which a P(VDFTrFE) film was bonded to the extended gate of a metal-oxide- a silicon wafer using standard NMOS process technology. This technique is compatible with IC fabrication processes and enables the integration of active elements with the transducer element on the same chip. The integrated structure eliminates long leads between the transducer and the amplifier and makes the net transducer-amplifier detectivity superior to the ceramic transducer-amplifier system. The devices are then encapsulated with Rho-C rubber which results in good acoustic impedance to that of water. The acoustic sensing performance was carried out in a pulse tube over a frequency range from 4 to $10 \mathrm{kHz}$. The experimental results along with the theoretical prediction by Mason equivalent circuit will be presented.

\title{
Session 4aSCa
}

\section{Speech Communication: Auditory Function and Segmental Perception (Poster Session)}

\author{
Ann R. Bradlow, Chair \\ Department of Linguistics, Northwestern University, 2016 Sheridan Road, Evanston, Illinois 60208
}

Contributed Papers

\begin{abstract}
All posters will be on display from 8:30 a.m. to 11:00 a.m. To allow contributors an opportunity to see other posters, contributors of odd-numbered papers will be at their posters from 8:30 a.m. to 9:45 a.m. and contributors of even-numbered papers will be at their posters from 9:45 a.m. to 11:00 a.m.
\end{abstract}

4aSCa1. Auditory temporal resolution and speech performance in cochlear implant users. Qian-Jie Fu (House Ear Inst., 2100 W. Third St., Los Angeles, CA 90057)

It has been widely assumed that speech recognition depends, among other factors, on the auditory temporal resolution function. The present study was conducted to investigate the relationship between auditory temporal resolution and speech performance in nine Nucleus-22 cochlear implant (CI) listeners fitting with the SPEAK strategy. A modulation detection threshold task was used to evaluate auditory temporal resolution. Modulation detection thresholds were measured as a function of current levels. Results showed some marked difference in the temporal modulation function across CI users. Some patients showed increased modulation thresholds across the entire dynamic range while others showed thresholds asymptoted as low as $30 \%$ of the dynamic range. There is a strong correlation between consonant recognition scores and the mean modulation detection thresholds (across the entire dynamic range) among CI users. The correlation between the mean modulation detection thresholds and vowel recognition scores was relatively weak but was still significant. The results suggest that phoneme recognition, especially consonant recognition, depends highly on the patients' capability to detect temporal modulation. One implication from the present results is that the high variability of speech performance is at least partly due to the differences in the temporal resolution of CI users. [Work supported by NIDCD.]

4aSCa2. Intelligibility of words by reduced-channel and cochlear implant listeners. Thomas Carrell, Kathy Shapley (Commun. Disord., Univ. of Nebraska, Lincoln, NE 68583), and Dawn Koch (Northwestern Univ., Evanston, IL 60208)

One of the differences in the way a cochlear implant, versus a normally functioning cochlea, delivers sound to auditory centers in the brain is that the implant delivers far fewer channels of frequency information. This experiment investigated how speech intelligibility in cochlear implant
(CI) listeners may be related to reduced frequency resolution as opposed to other factors. A closed-response set Modified Rhyme Test was administered to CI listeners [House et al., J. Acoust. Soc. Am. 37 (1965)] using naturally produced words spoken in isolation. The stimuli presented to the listeners without implants were a reduced-channel (RC) version of exactly the same stimuli, which were filtered and processed in software in a manner similar to the hardware method described by Shannon [Science $\mathbf{2 7 0}$ (1995)]. The effect of the signal processing was to divide each word into four frequency channels for the first group of RC listeners and into eight channels for the second group. Results indicated final phoneme identification appeared to be more difficult for CI listeners than for four- or eightband RC listeners. Results were more variable for phonemes in the initial position. CI listeners were better than both four- and eight-band listeners on affricates and better than eight-band listeners for weak fricatives.

4aSCa3. Speech perception, production, and intelligibility improvements in vowel-pair contrasts among adults who receive cochlear implants. Jennell Vick, Harlan Lane, Joseph Perkell, Melanie Matthies (Speech Commun. Group, Res. Lab. of Electron., Rm. 36-511, M.I.T., 50 Vassar St., Cambridge, MA 02139), John Gould, and Majid Zandipour (Res. Lab. of Electron., Cambridge, MA 02139)

This study investigates relations among speech perception, speech production, and intelligibility in postlingually deaf adults who receive cochlear implants (CI). Measures were made for seven vowel pairs that neighbor in acoustic space from eight postlingually deafened adults, preand postimplant. Improvements in a speaker's production, perception, and intelligibility of a given vowel contrast tended to occur together. Subjects who produced vowel pairs with reduced contrast in the preimplant condition (measured by separation in the acoustic vowel space) and who showed improvement in their perception of these contrasts postimplant (measured with a phoneme identification test) were found to have improved production contrasts post-CI. These enhanced production contrasts 
were associated with enhanced intelligibility, as measured from responses of a group of normal-hearing listeners. The results support the hypothesis that the implant user's improving speech perception contributes, at least in part, to that speaker's improving speech production. [Work supported by the NIDCD, NIH.]

4aSCa4. Channel interaction and speech processing strategies for cochlear implants. Ginger S. Stickney, Philip Loizou, Lakshmi Narayan Mishra, Peter F. Assmann (Univ. of Texas at Dallas, Box 830688, Richardson, TX 75083-0688), Robert V. Shannon (House Ear Inst., Los Angeles, CA 90057-1902), and Jane M. Opie (InSonus Medical, Inc., Newark, CA 94560)

A limitation of multichannel cochlear implants is that electrical channel interactions can arise when multiple electrodes are stimulated simultaneously. Channel interaction can be reduced by decreasing current levels delivered to each electrode, through improved electrode positioning and design, or by using speech processing strategies that stimulate electrodes sequentially. The goal of the present study was to examine the relationship between channel interaction and speech recognition for several speech strategies varying in the number of electrodes stimulated simultaneously. Based on previous research, susceptibility to channel interaction is expected to vary as a function of electrode configuration. Thus patients with strong susceptibility to channel interaction will produce higher speech recognition scores for sequential as opposed to simultaneous speech strategies. Speech strategy performance was evaluated with acute listening trials where patients identified consonants and vowels with each of the following speech strategies, listed in order from sequential stimulation to fully simultaneous stimulation: Continuous Interleaved Sampler (CIS), Paired Pulsatile Sampler (PPS), Hybrid Analog Pulsatile (HAP), and Simultaneous Analog Stimulation (SAS). Preliminary results showed that a greater degree of channel interaction was associated with the highest speech recognition scores for CIS, midrange scores for HAP, and the lowest scores for SAS. [Work supported by NIH.]

4aSCa5. Cochlear-implant effects on sibilants. Melanie Matthies (Dept. of Commun. Disord., Boston Univ., 635 Commonwealth Ave., Boston, MA 02215, matthies@bu.edu), Joseph Perkell, Jennell Vick, and Majid Zandipour (MIT, Cambridge, MA 02139)

To investigate sibilant production in persons with cochlear implants, this study compared acoustic analyses of three sets of speech stimuli: (1) A synthesized continuum of sibilants from "sa" to "sha" was constructed where the characteristics of the sibilant noise as well as the F2 and $F 3$ transitions were varied. (2) The synthesized speech was processed through a cochlear-implant simulation program [cf. Shannon et al., Science 270, 303-304 (1995)]. (3) Longitudinally recorded speech production data were obtained from postlingually deafened adults who had received cochlear implants. Formant frequencies and amplitudes were obtained at midvowel and at vowel onset. The amplitude in the sibilant at the frequency of vowel $F 3$ was measured, as were the spectral characteristics of the sibilant noise for all three sets of speech. Judgments about the identity and quality of the synthesized sibilants were obtained from a panel of listeners with normal hearing. Correlations indicated that the selected acoustic cues were highly salient to the listeners for both the unprocessed and cochlear-implant-simulated sibilants. Observed improvements in sibilant production from pre-CI to post-CI are likely due to the improved availability of these salient cues for auditory feedback and selfmonitoring. [Work supported by MURI Z883402, NIDCD DC03007.]

4aSCa6. Feature extraction in human speech recognition. J. B. Allen, M. Furst, L. Saul, and M. Rahim (AT\&T Labs, Florham Park, NJ 07932)

In 1955 George Miller and Patricia Nicely explored the human speech code and discovered that a five-channel feature code is used to extract phonemes from speech. Their mutual information analysis of the confu- sion matrices of filtered noisy speech showed that the five channels were virtually independent of each other, accounting for the robustness of speech communication quantified by Miller, Heise, and Lichten in 1951. While the 1955 analysis was a major step forward, it did not lead to practical results, since there are no known methods for extracting these features from the speech. This weakness is the basis of the present research program, to define the signal processing required to robustly extract features from narrow bands of speech. Our emphasis is to work in narrow bands, starting from critical bands. In this paper we discuss the first step of this process, which is to measure (a) the jnd for the detection of speech in noise and (b) the jnd for residue pitch given narrow bands of speech in noise. We have been investigating these features with the ultimate goal of trying to build a speech recognition machine based on distinctive feature signal processing.

4aSCa7. Vowel perception in varied symmetric CVC contexts. Jim Talley (Dept. of Linguist., Univ. of Texas, Austin, TX 78712)

In the three-decade-long debate over static versus dynamic specification of vowels, perceptual studies in which subjects are tasked with identifying naturally spoken vowels under various ablation conditions have been a mainstay. While not directly producing an understanding of how humans go about recognizing this major subclass of phones, this type of study [e.g., Strange, Jenkins, and Johnson, J. Acoust. Soc. Am. 74, 695705 (1983)] has provided compelling results which must be accounted for in any successful theory of vowel perception. This paper presents results from yet another perceptual study of human vowel identification under ablation conditions. This study uses CVC syllables spoken rapidly by three male speakers in a carrier sentence. Syllables consist of ten American English vowels in each of four consonantal contexts (b_b, d_d, g_g, and $\mathrm{h} \_d$ ). The conditions studied are silent centers (SC), centers only (CO), and the control condition (full). A very robust hierarchy of full $>\mathrm{CO}>\mathrm{SC}$ is found. Consonantal contexts also have a clear ordering ( $h \_d>b \_b>d \_d>g \_g$ ) with respect to the ease with which they are perceived. Interesting interactions between vowels and their contexts are also evident.

4aSCa8. Vowel formant discrimination in natural speech. Chang Liu and Diane Kewley-Port (Dept. of Speech and Hearing Sci., Indiana Univ., Bloomington, IN 47405, liuchang@indiana.edu)

Vowel formant discrimination in syllables, phrases, and sentences was measured for nearly natural speech resynthesized by STRAIGHT [Kawahara et al., Speech Commun. 187-207 (1999)]. The purpose of experiment 1 was to validate the use of STRAIGHT to modify formants by comparing vowel discrimination in formant-synthesized and -resynthesized isolated vowels. The resulting DLs for $F 1$ and $F 2$, calculated in barks, were the same as previously reported. In experiment 2 , DLs were measured in natural speech for $F 1$ and $F 2$ for the vowels /ih, eh, uh, ae/. Different phonetic contexts included syllables, phrases, and sentences, as well as sentences with the addition of an identification task. Results showed that longer phonetic context was not a strong factor in degrading DLs, although performance for syllables was significantly better than for sentences. The addition of the identification task did not have a significant effect on vowel discrimination. In comparison to the formant-synthesized speech in Kewley-Port and Zheng [J. Acoust. Soc. Am. 106, 2945-2958 (1999)], vowel discrimination was similar for syllables, but significantly degraded for more complex contexts. However, the magnitude of the results suggests that vowel formant discrimination is similar for synthetic and natural speech in a variety of phonetic contexts. [Work supported by NIHDCD-02229.] 
4aSCa9. Extracting vowel characteristics from smoothed spectra. Jean-Sylvain Lienard (LIMSI-CNRS, B.P.133, 91403 Orsay Cedex, France, lienard@limsi.fr) and Maria-Gabriella Di Benedetto (Universita' di Roma La Sapienza, 00184 Rome, Italy)

The object of this study was to define vowel spectral features by means of a spectral representation making no specific reference to formants and fundamental frequency, though preserving vowel identity and extralinguistic factors such as effort and gender. This investigation complements the analysis in Lienard and Di Benedetto [J. Acoust. Soc. Am. 106, 411-422 (1999)] which used manually measured formants of the same data. The database consisted of isolated French vowels, uttered by male and female speakers, with different vocal efforts. The analysis was carried out by selecting one segment along the vowel corresponding to a joint maximum of stability and level. A spectral envelope, based on prominent spectral peaks selected by a frequency-domain masking function, was computed. The frequency axis was Bark-transformed. The envelope was then decomposed by successive smoothings (cepstral filtering). The output was a representation where vowels are characterized by the presence or absence of energy concentration at different frequency locations ("spectral bumps"). Spectral bumps, although related to formants, do not correspond to formants themselves; rather, they reflect an integrated information similar to what might be used by the perceptual system.

4aSCa10. Effect of masking of spectrally reduced speech with modulated noise. Angélique Grosgeorges, Frédéric Berthommier (Institut de la Commun. Parlée, 46, Av. F. Viallet, 38031 Grenoble Cedex, France), and Christian Lorenzi (LPE, 92774 Boulogne-Billancourt, France)

Accurate recognition performance can be obtained with spectrally degraded speech [R. V. Shannon et al., Science 270, 303-304 (1995)]. Part of this experiment was initially reproduced for $16 \mathrm{VCVCV}$ stimuli $(\mathrm{a} / \mathrm{C} /$ a/C/a) spectrally degraded and presented in quiet. Then, the contribution of low- and high-frequency amplitude modulations (AM) of speech and noise masker presented at 6-dB SNR was investigated, using a 2 factors/2 levels experimental design: The speech envelope was low-pass filtered at 10 or $500 \mathrm{~Hz}$; the masker was a white noise with low-frequency AM components $(<8 \mathrm{~Hz})$, which was sinusoidally amplitude modulated at $100 \mathrm{~Hz}$ or not. In addition, the low-frequency AM components of the masker were co-modulated across the four frequency bands or not. Compared to the scores obtained in quiet, a decrease of about $50 \%-75 \%$ in the transmitted information for place and voicing cues was observed in all conditions, and transmission of manner was dramatically suppressed. As expected, recognition of spectrally degraded speech was strongly affected by temporal masking, but the detrimental effects of noise were not uniform for all speech features, giving some insight about their respective temporal encoding. [Work supported by RESPITE.]

4aSCa11. Gross versus detailed spectral cues in spectrally distorted speech. Michael Kiefte) (Dept. of Linguist., Univ. of Alberta, Edmonton, AB T6G 2E7, Canada)

An experiment was designed to address the importance of gross versus detailed spectral features in the perception of stop-consonant place of articulation. This is done by presenting listeners with noise-vocoded stimuli in which the spectral resolution of the processed tokens is severely reduced. While it can be assumed that such a transformation will effectively eliminate detailed spectral features, such as burst-peak and formant frequencies, previous work has shown that this is not necessarily the case [M. Kiefte, J. Acoust. Soc. Am. 106, 2273 (1999)]. Nevertheless, it is shown that gross spectral features, such as spectral tilt and compactness, are better able to predict listeners' identifications of noise-vocoded stimuli. While this suggests that listeners attend primarily to gross spectral shape cues, which are assumed to be particularly robust in such distorted speech, it is also shown that detailed spectral features are better able to model listeners' responses to undistorted speech. [Work supported by SSHRC.] a) Now at the Dept. of Psychology, University of Wisconsin, Madison, WI.
4aSCa12. Long-term speech spectra for Taiwanese-Mandarin bilingual speakers. Chin-Hsing Tseng, Hsiao-Chuan Chen (Dept. of Special Education, Natl. Kaohsiung Normal Univ., Kaohsiung, Taiwan, ROC), and Shing Chen (Industrial Technol. Res. Inst., Hsin-Chu, Taiwan, ROC)

Audiologists and otologists in Taiwan often rely on the English normative data during the hearing aid gain prescription procedure. Thus, the measurement of long-term speech spectra for both Taiwanese and Mandarin was deemed a research priority. Average long-term rms 1/3-octave band speech spectra were generated for 60 Taiwanese-Mandarin speakers with gender, age, and language as the three major independent variables. The male speakers differed significantly from the females in the lowfrequency bands across languages and age groups. However, a slight gender difference in the $4000-\mathrm{Hz}$ band was observed only in the young adult group (aged 20-40) for both Taiwanese and Mandarin. Across languages, the elder group (aged 41-60) differed significantly in the low-frequency bands for both genders, but high-frequency differences existed only in the females. The overall spectra will provide a useful reference for hearing aid gain prescription in the Chinese-speaking communities. Cross-language comparisons can also shed insight on the speech communication processes. [Work supported by the Council of Labor Affairs.]

4aSCa13. Effects of spectral mismatch on sentence recognition for noise-band processors. John J. GalvinIII and Qian-Jie Fu (Dept. of Auditory Implants and Percept., House Ear Inst., 2100 W. 3rd St., Los Angeles, CA 90057)

For cochlear implant (CI) users, the cochlear insertion depth and limited spatial extent of the electrode array causes a mismatch between the frequency content of speech signals and the place of stimulation along the cochlea. To examine the effects of such a spectral mismatch on HINT sentence recognition in noise, five normal-hearing $(\mathrm{NH})$ listeners were tested using noise-band speech processors designed to simulate a variety of cochlear insertion depths. The speech reception threshold (SRT) was measured for two conditions: (1) analysis and carrier bands matched to the insertion depth, and (2) analysis bands fixed over a wide range while carrier bands varied according to insertion depth. In the matched condition, subjects' performance remained flat along the entire range of insertion depths; on average, the SRT was $5 \mathrm{~dB}$ lower than the mismatched condition, indicating that a frequency-limited, but spectrally matched processor provided more benefit. In the mismatched condition, performance was flat over most of the range of insertion depths and declined only for extremely basal or apical insertions. The results indicate that electrode insertion depth and the resulting spectral mismatch is probably not the limiting factor affecting speech recognition or contributing to the variability in performance observed among CI users.

4aSCa14. Effects of lexical and talker variability factors on spoken word recognition in native and non-native, normal and hearingimpaired listeners. Sumiko Takayanagi, Donald D. Dirks, Anahita Moshfegh, P. Douglas Noffsinger (UCLA School of Medicine, Los Angeles, CA 90095, takayana@ucla.edu; Natl. Ctr. for Rehab. Auditory Res., OR 97207, and West LA VAMC, Los Angeles, CA 90073), and Stephen A. Fausti (Natl. Ctr. for Rehab. Auditory Res., Portland, OR 97207)

The effects of lexical difficulty and talker variability on word recognition were examined in four groups of listeners: native English/normal hearing; native English/hearing impaired; non-native English/normal hearing; and non-native English/hearing impaired (hearing level matched to the native hearing impaired). Lexical difficulty was measured by the difference in performance to 75 lexically "easy" and "hard" words based on word frequency and Neighborhood Activation Theory [Luce and Pisoni (1998)]. The effect of talker variability was measured by the difference in performance between single and multiple talker (nine talkers) conditions. The familiarity of the 150 words was rated on a seven-point scale. An up-down adaptive procedure was used to determine the sound pressure 
level for $50 \%$ performance. Non-native listeners in both normal and hearing-impaired groups required a greater intensity for equal intelligibility than for the comparative native normal and hearing-impaired listeners. Results, however, showed significant effects of lexical difficulty and talker variability in all four groups. Structural equation modeling demonstrated that an auditory factor estimated by pure tone average, etc., accounts for four times more variance to performance than does a linguistic fluency factor measured by word familiarity ratings and native versus non-native status, however, the linguistic fluency factor is also essential to the model fit.

4aSCa15. Speaking clearly for learning-disabled children: Sentence perception in noise. Ann R. Bradlow (Dept. of Linguist., Northwestern Univ., 2016 Sheridan Rd., Evanston, IL 60208), Nina Kraus, and Erin Hayes (Northwestern Univ., Evanston, IL 60208)

This study sought to establish connections between two findings regarding speech perception in special populations. First, children with learning problems often have difficulty perceiving speech in noise. Second, naturally produced clear speech is more intelligible for adult hearingimpaired listeners. Given recent interest in the use of digital enhancement techniques in speech and language training procedures for learningdisabled children, we wanted to investigate whether naturally produced clear speech yields perception benefits for this population. We presented children with learning problems $(n=46)$ and normal children $(n=17)$ with sentences embedded in noise. Factors that varied were speaking style (plain versus clear) and signal-to-noise ratio (high versus low). Results indicated that: (1) children with learning problems had poorer sentencein-noise perception than normal children; (2) both groups had poorer perception with increasing noise levels; and (3) both groups benefited from naturally produced clear speech. There were no significant two- or threeway interactions between listener group, noise level, and speaking style. In combination with acoustic analyses of the plain and clear sentences, these findings have broad implications for our understanding of the speech perception deficits in this population, and the design of appropriate training procedures. [Work supported by NIH-NIDCD Grants DC 03762 and DC 01510.]

4aSCa16. Adaptive thresholds for spoken sentence materials. Theodore Bell (Dept. of Commun. Disord., King Hall B119, 5151 State University Dr., Cal State L.A., Los Angeles, CA 90032-8170), Richard Wilson (Quillien Veteran Affairs Medical Ctr., Mountain Home, TN), Jane Enrietto, and Carmen Morris (Cal State L.A., Los Angeles, CA 90032-8170)

Two experiments document the reliability of a speech discrimination test designed for use with an adaptive procedure to measure thresholds for sentences and words. Stimulus words were categorized as either high usage or low usage based on published indices of word usage, and also as either high confusability or low confusability depending on phonetic similarity to other words in the lexicon. The first experiment employed 20 young normal-hearing adults, each presented with two 20-item lists of sentences from each of the four lexical categories. One week later, the testing sequence was repeated. A five-factor ANOVA was employed using word usage, word confusability, list sample, and test-retest delay; an adaptive procedure, converging determined sentence thresholds in $\mathrm{dB}$ level. Test-retest thresholds, both immediate and delayed, were within 2 $\mathrm{dB}$ for all lists. There was a 1.5-dB difference between high- and lowusage words. A second experiment examined thresholds for groups of hearing-impaired individuals categorized by audiometric configuration and speech discrimination scores. Each subject group was presented two sentence lists varying in lexical characteristics based on the Neighborhood Activation Model. The difference between lexical items varied significantly with speech discrimination scores and audiometric configuration of the listeners. [Work supported by Veteran Affairs R\&D Contract V621P3664.]
4aSCa17. Co-modulation masking release (CMR) in consonant recognition. Bom Jun Kwon (Dept. of Auditory Implant and Percept., House Ear Inst., 2100 W. 3rd St., Los Angeles, CA 90057-1922)

In the psychoacoustic phenomenon of co-modulation masking release (CMR), detection of a target in a narrow-band noise is improved in the presence of a co-modulated flanking noise band. The present study investigates whether CMR could improve speech perception. Consonant recognition scores of bandlimited speech, 1200-2200 Hz, were measured under a modulated noise of the same bandwidth, and a second band of noise $(5000-7000 \mathrm{~Hz})$, which was either co-modulated or non-co-modulated with the first band. With a hypothesis that CMR improves discrimination of phonemes that are characterized by temporal cues, i.e., amplitude envelope, rather than spectral cues, two groups of consonants were selected for testing: stop consonants (similar temporal envelopes, but different spectra) and alveolar-dental consonants (similar spectra, but different temporal envelopes). Results agreed with the hypothesis: significantly higher recognition scores were obtained with the co-modulated noise band for alveolar-dental consonants, while the difference was marginal for stop consonants. This indicates that CMR can improve speech perception as well as psychophysical thresholds. Co-modulation in the flanking band seems to enable listeners to better " hear out" subtle temporal speech cues in noise, leading to better recognition. [Work supported by Quota scholarship and NIDCD.]

4aSCa18. Effects of filtering preceding noise on the intelligibility of voiced plosives. William A. Ainsworth (MacKay Inst. of Commun. and Neurosci., Keele Univ., Staffordshire ST5 5BG, UK) and Teresa Cervera (Univ. of Valencia, Spain)

The intelligibility of voiced plosives in continuous noise is greater than in noise which is coincident with the speech [W. A. Ainsworth and G. Meyer, J. Acoust. Soc. Am. 96, 687-694 (1994)]. It is suggested that this could be due to some adaptation process. In order to test this hypothesis, experiments have been performed to measure the intelligibility of voiced plosives in isolated syllables with different durations of preceding noise. The effects of the spectral shape of the noise have also been examined. It was found that, on average, $800 \mathrm{~ms}$ of band-stop filtered noise preceding the syllable increased the recognition rate of the plosives by $14 \%$, bandpass filtered noise by $12 \%$, low-pass filtered noise by $7 \%$, but high-pass filtered noise by only $3 \%$ compared with co-gated noise. The time constant of the adaptation process was estimated to be $165 \mathrm{~ms}$. [Work supported in part by EU TMR Network SPHEAR.]

4aSCa19. Across-frequency timing in speech perception: Effective processing of contrasting temporal speech patterns requires proper synchronous alignment. Eric W. Healy and Sid P. Bacon (Psychoacoustics Lab., Dept. of Speech and Hearing Sci., Arizona State Univ., P.O. Box 871908, Tempe, AZ 85287-1908, ewh@asu.edu)

Although narrow-band temporal speech patterns produce virtually no intelligibility when presented individually, a contrasting pair of such patterns can produce appreciable intelligibility [E. W. Healy, Dissertation, University of Wisconsin-Milwaukee (1998)]. In the current study, the role of across-frequency timing in speech perception was investigated using these contrasting temporal speech patterns which allow the strict isolation of across-frequency integration. Across-band timing was investigated by varying the synchrony of paired temporal patterns. Individual temporal patterns were created by amplitude modulating tones at 750 and $3000 \mathrm{~Hz}$ to follow the temporal fluctuations of corresponding 1/3-octave sentences. The pair of individually unintelligible narrow-band temporal patterns were mixed and presented to listeners either in temporal synchrony, or with offsets of $12.5,25,50$, or $100 \mathrm{~ms}$. High intelligibility scores in the aligned condition were reduced when as little as $12.5 \mathrm{~ms}$ of misalignment was introduced, and performance fell to values near zero at the largest misalignment. These results indicate that normal-hearing young listeners are relying heavily on across-channel timing information provided by the syn- 
chronous temporal patterns. Further work employing this technique may provide insight into the role of timing in the suprathreshold speech processing deficits of elderly or hearing-impaired listeners. [Work supported by NIDCD.]

4aSCa20. Discriminant analysis of acoustic cues to three Green Mong tones. Jean E. Andruski (ASLP, 581 Manoogian Hall, Wayne State Univ., Detroit, MI 48202)

Green Mong is a tone language with an inventory of seven contrastive tones. The tonal system of Green Mong incorporates both pitch and phonation-type distinctions, and appears to make use of many of the acoustic parameters which have been shown to correlate with phonationtype cross-linguistically. This study looks at three Green Mong tones that have similar pitch contours, but are distinguished by the distinctive use of breathy, creaky, and modal phonation. Acoustically, the three tones are distinguished to some degree on the basis of pitch, relative amplitude of lower and higher harmonics (H1-H2), duration, vowel quality, and voice onset time. Discriminant analysis is used to estimate the value of these different cues for classifying tokens into tone categories based on observed characteristics in tone production. Discriminant functions were generated using measurements from $270 \mathrm{CV}$ words produced by six speakers, for which every combination of three vowels $(/ \mathrm{i} /, / \mathrm{u} /, / \mathrm{a} /)$ five consonants, and three tones (breathy, creaky, modal) was attested. Results of the analyses will be presented, as well as results for classification of a separate, test set of $102 \mathrm{CV}$ tokens produced by the same six speakers.

4aSCa21. Relation of pitch glide perception and Mandarin tone identification. Terry L. Gottfried (Dept. of Psychol., Lawrence Univ., Appleton, WI 54912) and Devon Riester (Kenyon College, Gambier, OH 43022)

Second language (L2) learners differ considerably in their ability to learn new phonetic contrasts in the L2. For example, Gottfried and Suiter (1997) observed that many native speakers of American English were highly accurate in their identification of Mandarin Chinese tones, while others, with similar years of experience in learning Mandarin, were very poor. The current study compared listeners' identification of Mandarin tones with their ability to perceive the pitch direction of sine-wave glides. Sine waves of $400 \mathrm{~ms}$ with starting frequencies of 250 and $300 \mathrm{~Hz}$ were created with $0-$ to $50-\mathrm{Hz}$ sweeps up or down from the initial frequencies. Listeners who had no experience with Mandarin indicated whether the pitch went up, down, or remained the same. After testing on the sine waves, listeners identified the tone (high-level, mid-rising, low-dipping, and high-falling) of intact and silent-center (with only the initial and final portions) Mandarin /1/-vowel syllables. Analysis revealed that listeners with musical training performed significantly better on intact Mandarin syllables than nonmusic majors, but no difference was observed for silentcenters. Voice majors seemed to have a particular advantage. Significant correlations were also found between tests of glide perception and tone identification (ranging from 0.530 to 0.642 ).

4aSCa22. Effects of time reversal on consonant identification. Sneha V. Bharadwaj and Peter F. Assmann (School of Human Development, Univ. of Texas at Dallas, Box 830688, Richardson, TX 75083-0688, snehab@utdallas.edu, assmann@utdallas.edu)

Time-reversed speech has the same long-term amplitude spectrum as natural speech, but is largely unintelligible. To study the perceptual effects of disrupting the temporal integrity of formant transitions on consonant identification, CVC syllables were divided into brief, successive, nonoverlapping segments and each segment was time reversed. In experiment 1 ,
CVC syllables from two talkers were time reversed using 20, 40, 60, 80, or 100-ms temporal windows. The initial consonant was either $/ \mathrm{p} /, / \mathrm{t} /, / \mathrm{k} /$, $/ \mathrm{b} /, / \mathrm{d} /, / \mathrm{g} /, / \mathrm{s} /, / \mathrm{J} /, / \mathrm{f} /$, or $/ \mathrm{v} /$. The medial vowel was $/ \mathrm{i} /, / \mathrm{u} /$, or $/ \mathrm{s} /$, and the final consonant was $/ \mathrm{t} /$. Identification accuracy (12 listeners) showed a progressive decline from $99 \%$ for unmodified syllables to $63 \%$ for the 100 -ms window. Time reversal was less disruptive when syllables were presented in a natural carrier sentence, dropping to $75 \%$ for a 100 -ms time window. Experiment 2 replicated this pattern of findings with a larger sample of ten talkers. The effects of time reversal were not uniform across the 12 consonants, but showed highly disruptive effects on the identification of stops and relatively minor changes in fricatives. Overall, the results suggest that time reversal provides a useful method for quantifying the role of dynamic information in consonant perception.

4aSCa23. Analysis-by-synthesis and acoustic landmarks. Richard S. McGowan (CReSS LLC, 1 Seaborn Pl., Lexington, MA 02420)

In the 1950s and 1960s Ken Stevens and Morris Halle proposed a model for human speech recognition that incorporated analysis-bysynthesis. In the more recent past the notion of acoustic landmarks has arisen from the speech group at MIT as part of a model for human lexical access from features by human listeners. Here, it is proposed that these ideas are fruitful ones for incorporation into a method (constraint and refinement) for training a machine with its own vocal tract to imitate human speech, while learning the relation between articulation and acoustic output. The machine would be trained in stages starting with the attempts to imitate the sequence of relatively open articulations and closed articulations. Landmarks, based on acoustic criteria, would be placed near the peaks in the amplitude envelope, allowing the machine to constrain the type of articulatory activations. This constraining step would involve, initially, an overly restrictive set of phonotatic rules, such as all sequences are obstruent-vowel sequences. The refining step would be the analysisby-synthesis with the machine's vocal tract. As the stages progress, the machine would attend to more acoustic properties of the speech signal with different kinds of landmarks and increase the generality of the phonotatic rules.

4aSCa24. Auditory robustness and duration of vocalic cues. John J. Ohala and Alan C. Yu (Dept. of Linguist., 1203 Dwinelle Hall, Univ. of California, Berkeley, Berkeley, CA 94720-2650, ohala@socrates.berkeley.edu)

Building on an idea of Stevens [ICPhS (1980)] and Stevens and Keyser [Language (1989)], Lang and Ohala (LO) [ICSLP (1996)] hypothesized that the most commonly used features to differentiate vowels in languages would be those few that can be detected in a relatively short time. Other features could be used to augment the vowel inventory at the expense of requiring more time for their perception. They found support for this idea in a perceptual study of end-truncated English vowels. We conducted a similar experiment on the perception of 18 contrasting vowels in Cantonese including monophthongs and diphthongs and both rounded and unrounded front vowels. The stimuli were vowel fragments starting at $30 \mathrm{~ms}$ after an initial [s] and end truncated into pink noise at 20-ms intervals thereafter. Twenty Cantonese heard randomized sets of these stimuli and attempted to associate each with the vowel in 18 different key words symbolized by Chinese characters. The resulting confusion matrices of the shorter fragments revealed not only the expected high confusions between monophthongs and diphthongs but also between rounded and unrounded front vowels. The latter contrast, which is indeed used less in language's vowel inventories, must require more time to perceive, supporting the LO claim. 


\title{
Session $4 \mathrm{aSCb}$
}

\section{Speech Communication and Archives and History: Speech Communication Within the Acoustical Society of America}

\author{
Patricia A. Keating, Chair \\ Department of Linguistics, University of California, Los Angeles, 3125 Campbell Hall, Los Angeles, California 90095-1543
}

Chair's Introduction-11:10

\section{1:15}

4aSCb1. Speech communication within the Acoustical Society of America. Katherine Harris (Haskins Labs., 270 Crown St., New Haven, CT 06511), Peter Ladefoged (UCLA, Los Angeles, CA 90095), and Kenneth Stevens (MIT, Cambridge, MA 02139)

Speech communication has been an important field of the Acoustical Society from its earliest years. Harvey Fletcher, the first President of the Society, along with many colleagues at Bell Telephone Laboratories, provided new data and techniques for investigating speech. The invention of the sound spectrograph during World War II led to greater understanding of the physics of speech, and this in turn produced developments in speech synthesis, summarized through the mid 1980's by Klatt [J. Acoust. Soc. Am. 82, 737-793 (1987)]. Physiological, psychological and linguistic aspects of speech have also been much investigated within the Society.

\section{A NOTE ABOUT THE ASA HISTORY LECTURE SERIES}

In 1997, the ASA Committee on Archives and History conceived a plan for a series of invited lectures on each of the technical areas of the Society which would memorialize the significant achievements and milestones of each of its twelve technical committees and one interdisciplinary technical group during the first three quarters of the Society's first century.

With the cooperation of the technical committees, distinguished individuals are selected to review the history of their particular technical speciality and present a lecture which shows how that activity has developed and has contributed to the Society at large and to the broad field of acoustics as well. At the meetings in Columbus, Ohio and Atlanta, Georgia, the first four History Lectures were presented: Gabriel Weinreich on Musical Acoustics, Robert Beyer and David Blackstock on Physical Acoustics, Stanley Ehrlich on Engineering Acoustics and Ewart Wetherill on Architectural Acoustics. At each subsequent meeting two additional lectures will be scheduled including those in Noise and Speech Communication at this meeting in Newport Beach.

The invited lecturers have been asked to prepare a written manuscript of their lectures which will be published in a commemorative book for the 75th Anniversary of the Society to be celebrated in 2004. The Archives and History Committee and the individual technical committees/group welcome comments and suggestions on both the History Lecture Series and on the proposed ASA Diamond Anniversary Book. Volunteers to assist the committees would be most welcome too. Contact Henry Bass, Chair, Committee on Archives and History, pabass@ sunset.backbone.olemiss.edu 


\title{
Session 4aUW
}

\section{Underwater Acoustics and Signal Processing in Acoustics: Acoustic Time Reversal and Acoustic Communications}

\author{
David R. Dowling, Chair \\ Department of Mechanical Engineering and Applied Mechanics, University of Michigan, 2019 Autolab 2121, \\ Ann Arbor, Michigan 48109-2121
}

Chair's Introduction-8:10

\section{Contributed Papers}

$8: 15$

4aUW1. Time-reversal pulse compression in shallow water with a single-element source-receiver. Charles F. Gaumond and David Fromm (Acoust. Div. Naval Res. Lab., 4555 Overlook Ave. SW, Washington, DC 20375-5320)

A time-reversing, full-water-column, source-receiver array has been previously demonstrated to focus and time compress a field at the position of the source through the use of all the propagating modes of the water column [W. S. Hodgkiss et al., J. Acoust. Soc. Am. 105, 1597-1604 (1999)]. Simulations are presented that show the limitations of using a single-element echo repeater instead of a full-water-column array. The system consists of a source located at the center of a vertical line array (VLA) and a distant, single-element echo repeater (SEER). The echo field produced by the SEER has very limited vertical extent of time compression at the range of the source and VLA. This vertical extent can be extended with the use of received signals from a varying number of adjacent hydrophones. The tradeoffs involved with extending the compression are shown. The simulations are generated using RAM in a simple downward-refracting shallow-water environment. [Work supported by ONR.]

8:30

4aUW2. Broadband performance of a time-reversing array in shallow water. Karim G. Sabra and David R. Dowling (Dept. of Mech. Eng., Univ. of Michigan, Ann Arbor, Ann Arbor, MI 48109-2121, drd@engin.umich.edu)

Future underwater communication systems may be developed from the spatial and temporal focusing properties of a time-reversing array (TRA). These properties ensue from the interactions between the underwater environment and the TRA. In a shallow-water sound channel, the influence of the TRA element number $(6,12,20)$ and spacing $(5 \mathrm{~m}, 3 \mathrm{~m})$, and source-array range (1 to $40 \mathrm{~km}$ ) on the spatial focusing and temporal compression will be presented. Here, the results show that compromises are necessary. Short ranges produce better spatial focusing while longer ranges produce better temporal compression. In addition, theoretical results for the performance of the TRA in an underwater environment with an ambient noise field has been evaluated for a family of pulses that span the range from narrow- to broadband. The theory, which has been the subject of previous presentations, accounts for the number of array elements and the time-bandwidth product of the signal. Based on this theory and numerical experiments, we will first investigate the effect of the sound channel characteristics on the performance of broadband signals and then compare broadband signals to narrow-band signals for several sourcearray ranges. [Work sponsored by the Office of Naval Research.]
$8: 45$

4aUW3. Robust diagnosis for the focal stability in time-reversal acoustics. H. C. Song, W. A. Kuperman (Scripps Inst. of Oceanogr., Univ. of California, San Diego, La Jolla, CA 92093-0238), T. Akal (SACLANT Undersea Res. Ctr., 19138 La Spezia, Italy), W. S. Hodgkiss, S. Kim, and G. Edelmann (Univ. of California, San Diego, La Jolla, CA 92093-0238)

For a time-independent medium, one can use stored probe pulses to focus on a specific location in time-reversal acoustics. However, the temporal variability of the ocean is expected to limit such a procedure. In the earlier low-frequency $(445 \mathrm{~Hz})$ experiments, we found that probe pulses up to 1 week old still produced a significant focus at the original probe source location. In the recent high-frequency $(3500 \mathrm{~Hz})$ experiments, however, stability of the focus was limited to less than an hour or even a few minutes, depending upon the oceanographic variability since the acoustic wavelength is much smaller than the one for $445 \mathrm{~Hz}$. A quick and robust diagnosis for the focal stability appears to be the modal group velocity versus phase velocity (gp curves) derived from the sound-speed profile, where the phase speed is directly related to the mode number and the slopes of the curves separate different groups of modes. In this paper, we investigate the stability and scintillation of the focus observed during the May 2000 time-reversal experiment using the gp curves.

9:00

4aUW4. Spatial resolution of time reversal arrays in shallow water. Seongil Kim, Geoffrey F. Edelmann, William S. Hodgkiss, William A. Kuperman, Hee Chun Song (Scripps Inst. of Oceanogr., Univ. of California, San Diego, La Jolla, CA 92093-0238), and Tuncay Akal (SACLANT Undersea Res. Ctr., 19138 La Spezia, Italy)

A series of time reversal (phase conjugation) experiments was performed in shallow water including a range-dependent slope environment. Time reversal arrays implemented with center frequencies of 445 and $3500 \mathrm{~Hz}$ achieved sharp focal regions up to ranges of 30 and $13 \mathrm{~km}$, respectively, in $110-130 \mathrm{~m}$ shallow water. Analysis with an image method indicates that the focal size approaches the diffraction limit of an array for given waveguide conditions, i.e., waveguide geometries and attenuation. The measured focal size has implications for the maximum achievable resolution of matched field processing, which is a computational implementation of the phase conjugation process. [Work supported by ONR.] 
4aUW5. Steering nulls in TRM with distortionless response at focal location-Theory and simulation. J. S. Kim, W. A. Kuperman, and H. C. Song (Scripps Inst. of Oceanogr., Univ. of California, San Diego, La Jolla, CA 92093, jkim@mpl.ucsd.edu)

The time-reversal mirror uses the received signal from a probe source to refocus the signal at the probe source location by back-propagating the time-reversed signal. In this study, a method to place a null at an arbitrary position while maintaining a distortionless response at the probe source location, assuming that the transfer function is known at that point, is proposed and demonstrated via simulation in Pekeris ocean waveguide. It is further shown that the null can be placed in the vicinity of probe source at the same depth using the theory on the invariants of ocean waveguide, without any environmental information. The approach is based on the adaptive weighting, of which formulation is a classical minimization problem with a constraint known as the minimum variance with distortionless response. The proper weighting for an array is applied before backpropagation. Finally, it is demonstrated that the null can be placed at the probe source location.

\section{9:30}

4aUW6. Applying the time-reversal operator to detection in a shallow-water environment. David Fromm and Charles F. Gaumond (Acoust. Div. Naval Res. Lab., 4555 Overlook Ave. SW, Washington, DC 20375-5320)

The decomposition of the time-reversal operator (DORT) is a method of isolating scatterers with a multiple-source/multiple-receiver system [C. Prada et al., J. Acoust. Soc. Am. 99, 2067-2076 (1996)]. A series of simulations is shown that elucidates the resolution of a DORT system in a reverberant, shallow-water environment. The system consists of vertical line arrays of sources and receivers in monostatic and vertically bistatic configurations. Two basic resolution problems are considered: the ability to discriminate two scatterers in the water column and the ability to detect a single scatterer in the presence of bottom clutter. The performance of the DORT system is shown relative to array configuration, location of scatterer in the water column, and the level and type of bottom clutter. [Work supported by ONR.]

\section{9:45}

4aUW7. Forward-scatter barrier with a time-reversal mirror. W. A. Kuperman (Scripps Inst. of Oceanogr., Univ. of California, San Diego, La Jolla, CA 92093-0701), T. Akal (SACLANT Undersea Res. Ctr., 19138 La Spezia, Italy), W. S. Hodgkiss, S. Kim, G. Edelmann, and H. C. Song (Univ. of California, San Diego, La Jolla, CA 92093-0238)

The classic difficulty in constructing an acoustic trip line barrier is that the forward-scattered field from the target must be extracted from the (usually) much more intense direct blasting arriving beam, i.e., "looking into the sunlight effect." During the time-reversal experiment conducted in May 2000, we investigated the forward-scatter barrier concept using a six-element transponder at $65-\mathrm{m}$ depth drifting along with a ship, which traversed the trip line between two moored vertical arrays separated by 5 $\mathrm{km}$ in $110-\mathrm{m}$ water depth. A $10-\mathrm{ms} \mathrm{cw}$ pulse from a probe source (PS) located at the bottom of the vertical receive array (VRA) is received and time-reversed at the source/receive array (SRA). The transmitted timereversed signal is then refocused at the PS location of the VRA. This time-reversed signal is also captured by the transponder simulating the forward-scatter target and is retransmitted to the VRA with various amplitudes simulating different forward-scatter target strengths. With an appropriate time delay, the main blast and the transponder signal arrived at the VRA simultaneously. For comparison, one-way broadside transmissions were also made.

\section{0:15}

4aUW8. Underwater acoustic communication by passive phase conjugation: Theory and experiment. Darrell R. Jackson, Daniel Rouseff, Warren L. J. Fox, Christopher D. Jones (Appl. Phys. Lab., College of Ocean and Fishery Sci., Univ. of Washington, Seattle, WA 98105, rouseff@apl.washington.edu), James A. Ritcey (Univ. of Washington, Seattle, WA 98105), and David R. Dowling (Univ. of Michigan, Ann Arbor, MI 48109)

A new method for coherent underwater communication called passive phase conjugation is evaluated. The technique takes its name because of conceptual similarities to active phase conjugation methods that have been demonstrated in the ocean [Kuperman et al., J. Acoust. Soc. Am. 103, 25-40 (1998)]. In contrast to active techniques, however, the array in passive phase conjugation need only receive. This makes the method plausible for scenarios where spatially compact sources might be communicating to a distant receive-only array. Compared to other approaches for coherent communication, the computational burden is low allowing the method to be evaluated in the field in nearly real-time. Results from an experiment conducted in Puget Sound near Seattle in May 2000 are reported. Various modulation schemes and array geometries were employed. Measurements were made at several ranges and water depths in a rangedependent environment. [Work supported by ONR.]

\section{0:30}

4aUW9. Self-equalization communications using a time-reversal mirror. G. Edelmann (Scripps Inst. of Oceanogr., Univ. of California, San Diego, La Jolla, CA 92093-0238), T. Akal (SACLANT Undersea Res. Ctr., 19138 La Spezia, Italy), W. S. Hodgkiss, S. Kim, W. A. Kuperman, and H. C. Song (Univ. of California, San Diego, La Jolla, CA 92093-0701)

A time-reversal mirror (TRM) was used to transmit self-equalization communication sequences in both range-independent and range-dependent environments. The TRM consisted of 29 source/receive elements centered at $3.5 \mathrm{kHz}$. A 2-ms pure-tone pulse was transmitted from a probe source at a range of $10 \mathrm{~km}$ from the TRM. A vertical receive array with 32 elements was colocated with the probe source to measure the spatial resolution of the TRM focus and bit error rate. The communication sequences were encoded using BPSK and higher-order phase-shift keying. As a comparison, single-source and broadside communication transmissions were also made.

\section{$10: 45$}

4aUW10. Using spatial coherence to improve the performance of underwater acoustic communications. T. C. Yang (Naval Res. Lab., Washington, DC 20375) and Martin Siderius (SACLANTCEN, La Spazia, Italy)

Phase-shifted keying modulation schemes offer an efficient use of signal power for a given bandwidth in a communication channel. Successful communication often requires multichannel data (spatial diversity) to combat the signal fading due to random fluctuations in the channel. For each channel, a signal-to-noise ratio (SNR) of $>10 \mathrm{~dB}$ is often required to estimate the channel impulse response for proper placement of the tap coefficients. Array beamforming can be used to improve the SNR, but when to use spatial diversity or beamforming is an issue long debated but not yet satisfactorily answered. It is shown with at-sea data that an effective use of beamforming versus spatial diversity depends on the phone spacing relative to the signal coherence. Beamforming requires the signals to be correlated between the sensors, whereas spatial diversity requires the opposite. Neither can fulfill the job of the other. By choosing phones according to spatial coherence and combining beamforming with diversity, bit error rates of the order of $\sim 0.001$ were demonstrated using Advent 99 data over a period of $\sim 9$ hours with an SNR as low as $\sim 1 \mathrm{~dB}$ at the phone level. [This work is supported by the Office of Naval Research and SACLANTCEN.] 


\section{1:00}

4aUW11. Analytic performance evaluation of coherent equalizers in time-varying acoustic channels. James C. Preisig (Dept. of Appl. Ocean Phys. and Eng., Woods Hole Oceanogr. Inst., Woods Hole, MA 02543)

Coherent equalization is one of the primary methods of implementing high-speed acoustic communications systems in underwater environments. This environment is often characterized by extended delay and Doppler spreads with different Doppler characteristics for the different delay taps of the time-varying impulse response. An analytic technique is presented which enables the evaluation of the performance of least-squares-based coherent equalizers in such channels. The technique is based upon statespace formalisms developed in the Kalman filtering literature. Comparisons between predicted and simulated equalization performance for realistic channel models are presented.

\section{1:15}

4aUW12. Guidelines for underwater acoustic data telemetry system design. Geoffrey Edelson, Francine Menas, and Michael Richman (Advanced Systems, Sanders, A Lockheed Martin Co., P.O. Box 868, Nashua, NH 03061-0868)
The use of acoustic communications in any number of projected applications requires trade-offs within the realm of technologies currently available and those projected for the future. Analyses must be performed to predict the performance, strengths, and limitations of competing approaches. Because underwater acoustic data telemetry can be viewed as a union of sonar and communications, it suffers from the same environmental degradation as sonar and communications systems. Efforts undertaken to date have shown that system performance is dictated by received signalto-noise ratio and multipath complexity, spread, and rate-of-change. Comparisons between different signaling technologies can be made using a variety of measures of effectiveness. These metrics provide a detailed structure for the evaluation of the performance envelope as a function of environmental and platform conditions. We have compiled published results on a large selection of modems in terms of range-rate product, bandwidth efficiency, range, frequency, and power. Based on these compiled results and on simple physical models, design rules-of-thumb have been derived to ensure that proposed system designs have communication requirements that are reasonable and robust. While these rules may seem somewhat conservative, the sample points reported in the literature may not have been achieved reliably or more than once. 


\section{Meeting of the Standards Committee Plenary Group}

\section{ORGANIZATION OF STANDARDS COMMITTEE PLENARY GROUP MEETING}

-S1 ACOUSTICS-U.S. Technical Advisory Group (TAG) for IEC/TC 29 Electroacoustics and ISO/TC 43 Acoustics

- S2 MECHANICAL VIBRATION AND SHOCK-U.S. Technical Advisory Group (TAG) for ISO/TC108 Mechanical Vibration and Shock

-S3 BIOACOUSTICS-U.S. Technical Advisory Group (TAG) for ISO/TC 43 Acoustics, IEC/TC 29 Electroacoustics, and ISO/TC 108/SC4 Human Exposure to Mechanical Vibration and Shock

-S12 NOISE-U.S. Technical Advisory Group (TAG) for ISO/TC 43/SC1 Noise

The meeting of the Standards Committee Plenary Group will precede the meetings of the Accredited Standards Committees $\mathrm{S} 1, \mathrm{~S} 3$ and S12, to take place in the following sequence on the same day:

$\begin{array}{lll}\text { S12 } & \text { 9:45 a.m. to 12 noon } & \text { California Salon 1 } \\ \text { S3 } & \text { 2:00 p.m. to 3:30 p.m. } & \text { Yawl Room } \\ \text { S1 } & \text { 3:45 p.m. to 5:00 p.m. } & \text { Yawl Room }\end{array}$

Discussion at the Standards Committee Plenary Group meeting will consist of national items relevant to all S Committees, plus a review of the international standardization (U.S. TAG) activities including reports on recent meetings and planning for forthcoming meetings.

Members of S2 on Mechanical Vibration and Shock (and U.S. TAG for ISO/TC 108 and five of its Subcommittees, SC1, SC2, SC3, SC5, and SC6) are also encouraged to attend the Standards Committee Plenary Group meeting even though the S2 meeting will take place on day earlier, on Wednesday, 6 December 2000, at 9:00 a.m.

The U.S. Technical Advisory Group (TAG) Chairs for the various international Technical Committees and Subcommittees under ISO and IEC, which are parallel to S1, S2, S3 and S12 are as follows:

\begin{tabular}{|c|c|c|}
\hline U.S. TAG Chair/Vice Chair & TC or SC & U.S. TAG \\
\hline \multicolumn{3}{|l|}{ ISO } \\
\hline $\begin{array}{l}\text { P. D. Schomer, Chair } \\
\text { H. E. von Gierke, Vice Chair }\end{array}$ & ISO/TC 43 Acoustics & $\mathrm{S} 1$ and $\mathrm{S} 3$ \\
\hline $\begin{array}{l}\text { P. D. Schomer, Chair } \\
\text { H. E. von Gierke, Vice Chair }\end{array}$ & ISO/TC 43/SC1 Noise & S12 \\
\hline D. D. Reynolds, Chair & ISO/TC 108/SC4 Human Exposure to Mechanical Vibration and Shock & S3 \\
\hline D. J. Evans, Chair & ISO/TC 108 Mechanical Vibration and Shock & S2 \\
\hline $\begin{array}{l}\text { R. H. Mehta, Chair } \\
\text { K. Won, Vice Chair }\end{array}$ & ISO/TC 108/SC1 Balancing, including Balancing Machines & S2 \\
\hline A. F. Kilcullen, Chair & $\begin{array}{l}\text { ISO/TC 108/SC2 Measurement and Evaluation of Mechanical Vibration } \\
\text { and Shock as Applied to Machines, Vehicles and Structures }\end{array}$ & S2 \\
\hline D. J. Evans, Chair & ISO/TC 108/SC3 Use and Calibration of Vibration and Shock Measuring Instruments & S2 \\
\hline $\begin{array}{l}\text { R. L. Eshleman, Chair } \\
\text { R. F. Taddeo, Vice Chair }\end{array}$ & ISO/TC 108/SC5 Condition Monitoring and Diagnostics of Machines & S2 \\
\hline G. Booth, Chair & ISO/TC 108/SC6 Vibration and Shock Generating Systems & S2 \\
\hline IEC & & \\
\hline V. Nedzelnitsky, U.S. TA & IEC/TC 29 Electroacoustics & $\mathrm{S} 1$ and $\mathrm{S} 3$ \\
\hline
\end{tabular}




\title{
Meeting of Accredited Standards Committee (ASC) S12 on Noise
}

P. D. Schomer, Chair S12, and Chair, U.S. Technical Advisory Group (TAG) for ISO/TC 43/SC1, Noise 2117 Robert Drive, Champaign, Illinois 61821

R. D. Hellweg, Vice Chair, S12

Compaq Computer Corp., Acoustics Lab, Mechanical Engineering Group, MRO1-3/D3, 200 Forest Street, Marlborough, Massachusetts 01752

H. E. von Gierke, Vice Chair, U.S. Technical Advisory Group (TAG) for ISO/TC 43/SC1, Noise 1325 Meadow Lane, Yellow Springs, Ohio 45387

Accredited Standards Committee S12 on Noise. Working group chairs will report on their progress for the production of noise standards.

Scope of S12: Standards, specifications and terminology in the field of acoustical noise pertaining to methods of measurement, evaluation and control; including biological safety, tolerance and comfort and physical acoustics as related to environmental and occupational noise.

\section{Session 4pAAa}

\section{Architectural Acoustics: The Technical Committee on Architectural Acoustics Vern O. Knudsen Distinguished Lecture}

\author{
Angelo J. Campanella, Chair \\ Campanella Associates, 3201 Ridgewood Drive, Hilliard, Ohio 43026-2453
}

Chair's Introduction-2:30

4pAAa1. Electronic architecture: Historical perspectives, current thinking, and future implications for architectural acoustic design. Paul Scarbrough (134 Washington St., D205, Norwalk, CT 06854, paulscrbro@aol.com)

Few subjects in acoustics generate more controversy than electronic architecture, that is, the use of electronic tools to alter the acoustics of a performance space, church, rehearsal hall, or other facility. And yet, few advances in acoustics offer the promise of electronic architecture. This paper will trace the development of electronic architecture from the first tentative and highly creative experiments in the 1930s to the mature technologies that have evolved in recent years. What precipitated the development of electronic architecture? Who were the pioneers? How did the technology develop? And where do we stand today? Paralleling these technological advances has been an on-going philosophical debate. Is there ever a place for electronics in the concert hall or opera house? When and where should these systems be used? What obligations do acoustical design professionals have to performers? To the concert-going public? To the art of acoustical design? Finally, what does the continuing evolution of these techniques hold for the future of architectural acoustic design? 


\title{
Session 4pAAb
}

\section{Architectural Acoustics: Building Acoustics Test Standards}

\author{
Kenneth P. Roy, Chair \\ Innovation Center, Armstrong World Industries, P.O. Box 3511, Lancaster, Pennsylvania 17604 \\ Chair's Introduction-3:45 \\ Invited Papers
}

3:50

\begin{abstract}
4pAAb1. Low-frequency impact sound rating of floor systems. Alf Warnock (Inst. for Res. in Construction, Natl. Res. Council, Ottawa, ON K1A 0R6, Canada)

An extensive series of measurements of airborne and impact sound insulation on joist floors of various types was recently completed at NRC. About 190 floors with different joist types, subfloors, ceiling types, ceiling support systems, and type and thickness of sound absorber were constructed and sound insulation measured to frequencies lower than required by standards. The joist types comprised solid wood, wood trusses, I-joists, and steel joists. Glass, rock, and cellulose fiber sound-absorbing material were used. Three concrete slabs were included in the series and a few of the joist floors had concrete toppings installed. Impact devices used on the floors included the standard ISO/ASTM E492 tapping machine, a walker, a JIS 1419 tire impactor, and two experimental rubber balls. The impact measurements provide an extensive, consistent set for examining alternative impact tests and possible modifications to standard tests using the ISO tapping machine. Correlations among the sound levels from the different impactors show that levels from the ISO tapping machine correlate well with those from a walker at low frequencies, although the spectra are quite different in shape. An overview of the data and the prospects for a new test method and rating system will be presented.
\end{abstract}

\section{4:10}

4pAAb2. Impact sound rating of floor toppings. Alf Warnock (Inst. for Res. in Construction, Natl. Res. Council, Ottawa, ON K1A 0R6, Canada)

Resilient materials either alone or under a slab of material are commonly placed on top of a hard floor surface to reduce impact sound transmission to rooms below. It is often impossible to select among such floor toppings when they have been tested on different base floor assemblies and only composite impact sound insulation ratings are available. ISO 140-8 measures the improvement due to a floor topping when placed on a concrete slab. The improvement may then be used to estimate the impact sound insulation of floors incorporating concrete slabs. In the project to be described, reductions in impact sound level were measured for a variety of toppings on several different floor types. The measurements confirmed that the ISO procedure works well and that relatively small specimen areas can be used without serious error for evaluation of floor toppings. The measurements showed that these improvements may not be applied to joist floors with lightweight subfloors such as plywood, but are useful for joist floors with thin concrete toppings. ASTM is adopting this test protocol for concrete slabs and is working on a modification that will provide data useful for toppings on joist floors with only light wood subfloors.

\section{Contributed Papers}

\section{4:30}

4pAAb3. Evaluating a method for field measurement of enclosure noise reduction. Felix Z. Sachs and David C. Byrne (U.S. Army Ctr. for Health Promotion and Preventive Medicine, Aberdeen Proving Ground, MD 21010-5403)

Measurement of noise reduction for sound isolating enclosures in situ is often complicated by limited access to all enclosure surfaces and indeterminate reverberation conditions. Both laboratory and field noise reduction measurements were made on an audiometric enclosure. The laboratory measurements followed ASTM method E596 using a reverberation chamber. The field method consisted of placing a "calibrated" loudspeaker at $1 \mathrm{~m}$ from the enclosure surface, applying a broadband signal and measuring $1 \mathrm{~mm}$ from the enclosure surface as well as inside the enclosure. The speaker calibration consisted of mapping the sound levels at $1 \mathrm{~m}$ in front of the speaker cone in an anechoic chamber. The field noise reduction is estimated using the inside and outside sound measurements. Since the loudspeaker sound field is localized, a surface area adjustment for the field noise reduction is required. The adjustment factor was derived from the E596 noise reductions. The factor is shown to be related to the loudspeaker calibration. [The opinions or assertions contained herein are the views of the authors and are not to be construed as official or as reflecting the views of the Department of the Army or the Department of Defense.]

\section{$4: 45$}

4pAAb4. Flanking sound transmission in wood-framed construction. Trevor Nightingale, Robin Halliwell, and David Quirt (Inst. for Res. in Construction, Natl. Res. Council, Ottawa, ON K1A 0R6, Canada)

This paper presents results from the first phase of a project to characterize flanking sound transmission at wall/floor junctions in wood-framed constructions suitable for multifamily residential buildings. This phase of the study focused on the junction between a wood joist floor and a separating wall with two rows of $38 \times 89-\mathrm{mm}$ studs. The objective of the study was to identify details that simultaneously provide good noise control and fire resistance. This paper presents the experimental approach and key results from the acoustical measurements. In addition to comparing how five common fire control solutions for the floor/wall junction affect the airborne and impact sound transmission, several generic retrofit modifications to reduce the structural noise transmission were tested. Parameters studied included the floor-joist orientation (parallel or perpendicular to the 
separating party wall), and the type of subfloor (plywood or oriented strand board). The study has shown that suitable details can provide both adequate fire resistance and noise control. The technical approach provides a framework for extending beyond established laboratory measurement procedures, to predict performance in actual buildings.

\section{5:00}

4pAAb5. Polish standardization of acoustic parameters of buildings in connection with ISO standards. Iwonna Zuchowicz-Wodnikowska and Anna Izewska (Bldg. Res. Inst., Ksawerow Str. 21, 02-656 Warsaw, Poland)

One of the foundations of Polish standardization in building acoustics for many years has been to establish the Polish Norms, as EN ISO Standards translated into the Polish language. In recent time, following establishment of ISO 717, it became necessary to amend the Polish Standard
(PN-B-02151-3:1999) in which the required acoustic insulation were defined. The new quantities of air-borne noise insulation are adapted to two model types of noise spectrum: pink noise characteristic, e.g., for residential noise, and road traffic noise with a considerable content of acoustic pressure levels in low- and medium-frequency ranges. The standard provides the following basic rules to determine the apparent indices of acoustic insulation (depending on the model spectrum): $\mathrm{R}^{\prime} \mathrm{A} 1=\mathrm{R}^{\prime} \mathrm{W}+\mathrm{C}$ or $\mathrm{R}^{\prime} \mathrm{A} 2=\mathrm{R}^{\prime} \mathrm{W}+\mathrm{Ctr}$. The standard also includes an informative supplement describing the simplified computing method of the flanking transmission. The key advantage of this standard lies in its obligatory character, i.e., it must be adhered to in both the design as well as execution of buildings, and it ensures the basic standard of acoustic performance. To cater to the needs of the designers, the Acoustic Division of ITB prepared a classification of building products comprising the indicated standard for measurement or computation and the class symbol and the range of the single-number quantity.

\title{
Session $4 \mathrm{pAB}$
}

\section{Animal Bioacoustics: William Cummings Session on the Acoustics of Whales and Dolphins I}

\author{
D. Vance Holliday, Chair \\ BAE Systems, 4669 Murphy Canyon Road, Suite 102, San Diego, California 92123
}

Chair's Introduction-2:00

Invited Papers

4pAB1. First known study, identification, and characteristics of fin and blue-whale vocalizations in the Northeast Pacific with historical notes and anecdotes. William C. Cummings (Oceanographic Consultants, 5948 Eton Ct., San Diego, CA 92103, oshendbs@aol.com) and Paul O. Thompson (San Diego, CA 92103)

In 1964 we were tasked by the Navy to identify and describe low-frequency, high-level noise mainly in the 1/3rd-octave frequency band containing $20 \mathrm{~Hz}$. Due to AGC and other presently antiquated processing then used at Northeast Pacific sound surveillance stations (SOSUS), acoustical monitoring was often confounded for significant annual periods heightening concern during that era of Cold War. Using data from continuous recordings for Gordon Wenz's ambient noise studies, we characterized the causative individual sources mainly as trains of approximately 1 -sec pulsed pairs and as long series of about 20 -sec paired moans. We determined seasonality, noise acceleration, geographic variation, representative source levels, and identity of the sources which turned out to be whales. This paper describes methods and findings of several years' study to identify and describe this "noise" employing numerous expeditions by sea and aircraft, now a more than 30-year-old precedent effort for which the authors (or their colleagues whose contributions shall be specified) have never been fully recognized by the military or by contemporary scientists. Intermingled we include trivia, such as the fate of the invaluable Wenz recordings, and interesting accounts of simultaneous Northwest Atlantic investigations by Schevill, Watkins, and colleagues including the present first author.

\section{$2: 30$}

4pAB2. Characteristics of chorusing sounds of humpback whales wintering in waters off western Maui. Whitlow W. L. Au, Marc O. Lammers, Paul E. Nachtigall (Hawaii Inst. of Marine Biol., Univ. of Hawaii, P.O. Box 1106, Kailua, HI 96734), Joseph Mobely (Univ. of Hawaii-West O'ahu, Honolulu, HI), and William C. Burgess (Greenride Sci., Inc., Santa Barbara, CA)

The dominant biological sound in the waters of west Maui during the humpback whale winter season in Hawaii is the chorusing sounds of singing humpback whales. A portable data logger was used to record chorusing sounds during most of the 1998 season. Sounds were digitized for 4 min every $\frac{1}{2} \mathrm{~h}$ and the data were stored on a hard disk. The results showed a peak in the sound pressure level between mid-February and mid-March coinciding with the peak in the number of whales sighted by aerial surveys. Significantly higher levels of chorusing sounds were also recorded at night. Sound levels began to increase during sunset, raising to a peak near midnight and remained relatively high until sunrise, after which the levels progressively decreased to a minimum between 11:00 a.m. and 3:00 p.m. That more humpback whales appear to sing at night may reflect a switch to sexual advertisement as the primary male mating strategy at this time; it may also indicate that daylight and vision play key roles in the formation of competitive groups. It is suggested that the relative number of humpback whales may be estimated by monitoring changes in sound pressure levels. 
4pAB3. Three-dimensional localization of blue whales using matched-field processing techniques: Unusual behavior, unanswered questions. A. M. Thode (MIT, 77 Massachusetts Ave., Cambridge, MA 02139), G. L D'Spain, and W. A. Kuperman (Scripps Inst. of Oceanogr., San Diego, CA 92093)

Recently, matched-field processing (MFP) and global inversion methods were used to track the three-dimensional trajectories of six blue whales, during a 1996 experiment off San Miguel Island in California, conducted off the research platform FLIP, using a 48-element, tilted vertical hydrophone array. Once the animals' positions and the propagation environment were determined, source levels were obtained for individual animals, and potential propagation effects were removed from their sounds. Out of the six whales studied, one showed unusual behavior. Initially it swam directly towards FLIP, while producing a diverse set of calls that included three strong FM sweeps. It then fell silent, altered its course to avoid FLIP, and began calling again as it swam away. Its closest point of approach to FLIP coincided with the arrival of a powerful tidal bore. The maximum source levels estimated from this animal were $10 \mathrm{~dB}$ lower than those from the other animals, and the FM sweeps showed signs of a possible resonance, which remained even when possible propagation effects were removed. The ability to precisely position blue whales has raised interesting questions about baleen whale sound production, the purpose behind these sounds, and the potential association between feeding animals and internal waves. [Work sponsored by ONR.]

3:10

4pAB4. Seasonal contribution of mysticete vocalization to ambient noise in southern California waters. David A. Helweg (Code D351, SSC San Diego, 49620 Beluga Rd., San Diego, CA 92152, helweg@spawar.navy.mil)

Many species of baleen whales reside or migrate annually through California waters. During these times some species of vocalizing whales can be detected, identified, and tracked with SOSUS arrays. Historical research by W. Cummings documented strong seasonal changes in narrow-band ambient sound associated with then unidentified sounds. Contemporary research shows these sounds to be blue and fin whale vocalizations. The current study compares historical and contemporary narrow-band ambient measurements with respect to mysticete residency and movement through southern California waters. Seasonal peaks in ambient noise agree with earlier reports, but increased anthropogenic sound reduced the magnitude of the effect. Potential sources of changes in ambient noise and their effects on the whales are discussed. [Research supported by the Strategic Environmental Research and Development Program, Project CS-1082.]

3:30

4pAB5. Thirty years of Australian marine bioacoustics and the Cummings connection. Douglas H. Cato (Defence Sci. and Technol. Organisation, P.O. Box 44, Pyrmont, NSW 2009, Australia), Robert D. McCauley (Curtin Univ. of Technol., Bentley, Western Australia), Michael J. Noad, and Tracey Rogers (Univ. of Sydney, NSW 2006, Australia)

Marine bioacoustics research in Australian waters has developed for more than 30 years, and has revealed a wide diversity of sounds and acoustic environments. The number of researchers and the complexity of the experiments has increased over the years. In the early days we had little knowledge or experience, and so relied on advice and help from American researchers. Of those, Dr. William C. Cummings remains one of our greatest supporters. This paper reviews some of the main features of the Australian work and the Cummings influence. Some of the earliest work, on fish sounds, echoes his work of the 1960s. This has since developed substantially, and we are planning to apply methods developed by Cummings of combined video and acoustic observation to source identification. Humpback whale sounds have been studied continually for more than 20 years and have revealed the complex songs of similar overall structure to those of the northern hemisphere, but with some surprises. Recordings have been made of southern right whales and Antarctic seals, and this year we recorded a large set of diverse sounds from blue whales. This data set and visual / acoustic observations of humpback whales are being used in censusing.

3:50

4pAB6. Two independent sonar signal generators in the bottlenose dolphin: Physiologic evidence and implications. Ted W. Cranford (Dept. of Biol., San Diego State Univ., San Diego, CA 92166), Wesley R. Elsberry, Diane J. Blackwood (Texas A\&M Univ., College Station, TX), Jennifer A. Carr, Tricia Kamolnick, Mark Todd (Science Applications Intl. Corp., San Diego, CA), William G. Van Bonn, Donald A. Carder, Sam H. Ridgway (U.S. Navy Marine Mammal Prog., San Diego, CA), Devon M. Bozlinski, and Emily C. Decker (Univ. of San Diego, San Diego, CA)

Some indirect evidence exists for multiple sonar signal generators in odontocetes. Direct evidence was collected from two bottlenose dolphins by simultaneously measuring and digitizing internal nasal cavity pressure, nasal tissue motion, and acoustic pressure. Small catheters measured pneumatic pressure changes at the same depth within each bony nasal passage. A high-speed video endoscopy system revealed tissue motion at both pairs of phonic lips while two hydrophones measured acoustic pressure during biosonar target discrimination. The records clearly demonstrate that acoustic pulses can be generated at the phonic lips on the left and right sides, independently or simultaneously. We have only seen the left phonic lips generate whistles. Air pressure in the bony nasal passages rises and falls together, even if the activity patterns at the two pairs of phonic lips are different. Increasing pulse repetition rate or sound pressure level, and whistle production, are all normally accompanied by increasing nasal air pressure. Whistle production apparently requires greater nasal air pressure than does pulse generation. Discovering multiple sonar sources in a dolphin's head should cause us to reinterpret previous findings and re-evaluate physiologic limits on pulse repetition rate, signal bandwidth, frequency composition, and projection beam geometry. [Work sponsored by Office of Naval Research.] 
4pAB7. High-frequency auditory filter shapes in an Atlantic bottlenose dolphin. David W. Lemonds, Whitlow W. L. Au, Paul E. Nachtigall, Herbert L. Roitblat (Marine Mammal Res. Prog., Hawaii Inst. of Marine Biol., Univ. of Hawaii, P.O. Box 1106, Kailua, HI 96734, lemonds@hawaii.edu), and Stephanie A. Vlachos (Sea Life Park Hawaii, Waimanalo, HI 96795)

High-frequency auditory filter shapes were calculated for a 20-year-old female Atlantic bottlenose dolphin (Tursiops truncatus). Thresholds were determined for tones of 40,60,80, and $100 \mathrm{kHz}$ masked by notched noise. Auditory filter shapes were determined by fitting the integral of the roex $(\mathrm{p}, \mathrm{r})$ filter shape to the functions relating masked tonal threshold to notch width. Filter shapes were found to be approximately symmetric at the moderate noise level used. Equivalent rectangular bandwidths of the auditory filters ranged from $16 \%$ of center frequency at $40 \mathrm{kHz}$ to $11 \%$ of center frequency at $100 \mathrm{kHz}$. There was very little change in the bandwidths of the filters between 60 and $100 \mathrm{kHz}$, indicating that relative tuning sharpness increases as a function of frequency in this range. Efficiency of processing after the periphery was found to be maximal at 40 and $60 \mathrm{kHz}$ (better than 12-dB SNR) and to decrease gradually above $60 \mathrm{kHz}$. The efficiency estimates allowed for the reanalysis of critical ratio data collected previously for the same animal; the bandwidth estimates from the present and earlier study were found to be in good agreement.

\section{4:30}

4pAB8. Foraging behavior of the Hawaiian spinner dolphin observed with active acoustics. Kelly Benoit Bird and Whitlow Au (Hawaii Inst. of Marine Biol., 46-700 Lilipuna Rd., Kaneohe, HI 96744)

The mesopelagic boundary community (MBC) in the Hawaiian Islands is an important food resource for many organisms, including spinner dolphins (Stenella longirostris). To understand the role of this community in the feeding ecology of spinner dolphins, spatial and temporal patterns in the density of the MBC and relative abundance of dolphins were characterized using acoustic sampling. A $200 \mathrm{kHz}$ echosounder modified to sample directly into a computer was used to survey the leeward coast of Hawaii from 2100 until 0400. Acoustic data were mapped in ArcView to show the distribution of both the predator and its prey. Individual echoes were color-coded by target strength to allow the separation of spinner dolphins and mesopelagic organisms. Dolphin species identification from active acoustics was confirmed using a combination of passive acoustics and visual observations. The MBC migrated horizontally, raising questions about the accepted (Norris) model of spinner dolphin foraging in Hawaii. Observations of spinner dolphins suggest a more complex foraging pattern, compatible with patterns observed in their food resource. Spinner dolphin foraging patterns closely matched the spatial patterns of their prey both horizontally and vertically. Patches exploited by dolphins were also significantly different than other patches measured under similar conditions.

\section{4:50}

4pAB9. Blue whale calls recorded in the Gulf of Alaska. Kathleen Stafford (CIMRS/COAS Hatfield Marine Sci. Ctr., Newport, OR 97365)

Blue whales (Balaenoptera musculus) were known to range throughout the North Pacific Ocean, including the Gulf of Alaska, before whaling activities greatly reduced their numbers. Despite repeated dedicated aerial and shipboard surveys in the Gulf of Alaska and off the Aleutian Islands, very few blue whales have been seen in these waters [NMFS, Mar. Fish. Rev. 61, 3843 (1999)]. Recent studies have shown the utility of acoustic methods for monitoring blue whale calls in the northeastern Pacific [cf. Thompson and Friedl, Cetology 45, 1-19 (1982); Stafford et al., Mar. Mamm. Sci. 15, 1258-1268 (1999); Watkins et al., Oceanography 13, 62-67 (2000)] but none of these have surveyed the Gulf of Alaska. In order to address the question of whether or not blue whales are still found in the Gulf of Alaska, six hydrophones were deployed there in October 1999. The first data set was recovered in May 2000. A preliminary analysis of these data showed that both northeastern [cf. Rivers, Mar. Mamm. Sci. 13, 186-195 (1997)] and northwestern [cf. Thompson and Friedl (1982)] Pacific blue whale calls were recorded on the hydrophones. 


\title{
Session $4 p A O$
}

\section{Acoustical Oceanography: Acoustic Measurements of Sediment Transport}

\author{
Alex E. Hay, Chair \\ Department of Oceanography, Dalhousie University, Halifax, Nova Scotia B3H 4J1, Canada \\ Chair's Introduction-1:00 \\ Invited Papers
}

1:05

4pAO1. TRIDISMA: An EU program for utilizing acoustics for measuring suspended sediment processes. Peter D. Thorne (Ctr. for Coastal and Marine Sci., Proudman Oceanographic Lab., Bidston Observatory, Bidston, Prenton CH43 7RA, UK), Jon J. Taylor (Env. Sci., Univ. of East Anglia, UK), Victor F. Humphrey, and Christopher E. Vincent (Bath Univ., UK)

In this paper the application of high-frequency acoustic remote sensing is discussed with respect to the development of an instrument capable of resolving the dynamics of the benthic boundary layer, in response to the interaction of waves and currents, using sound backscattered by the sediments entrained by the flow. Within the TRIDISMA project an acoustic instrument has been developed which combines three distinct approaches to the analysis of backscattered signals in order to extract information on flow structure and the response of the sediment bed to the overlying flow. The TRIDISMA instrument combines pulse coherent Doppler and acoustic cross-correlation techniques to the problem of extracting profiles of velocity, while the amplitude of the backscattered signal measured at three frequencies is used to investigate the response of the suspension field in terms of both size and concentration profiles. In the present paper we describe the hardware and software architecture of the prototype instrument and demonstrate the systems capabilities with results obtained from flume studies of waves, currents, and combined flows.

\section{$1: 30$}

4pAO2. Rake, a wave measuring benthic acoustic stress sensor. Archie T. MorrisonIII (McLane Research Labs., Inc., Falmouth Technology Park, 121 Bernard E. Saint Jean Dr., East Falmouth, MA 02536, atmorrison@mclanelabs.com) and Albert J. WilliamsIII (Woods Hole Oceanogr. Inst., Woods Hole, MA 02543)

The Rake is an acoustic travel time current meter designed to make spatially and temporally dense velocity profile measurements in the continental shelf wave bottom boundary layer. The high levels of bottom shear stress generated in this extremely thin, oscillatory, boundary layer play an important role in the entrainment of sediment into the water column and also enhance the turbulent dissipation of energy in overlying steady flows. The array of acoustic transducers measures the horizontal velocity vector with millimeter vertical resolution at up to 64 heights. A multiplexing transmit/receive circuit permits measurement of the vertical component of velocity with 5 to $10 \mathrm{~cm}$ vertical resolution using the same array. Complete velocity profiles can be acquired within a $40 \mathrm{~ms}$ window. Measurement error due to velocity averaging over the sample volume of a single acoustic axis has been shown to be negligible to within $1 \mathrm{~mm}$ of the bottom both by calculation and experiment. Laboratory calibrations and experiments have been conducted in steady and oscillatory flow. A month-long deployment of a field prototype took place in December of 1996. Data from these analyses and experiments will be presented. [The development of the Rake was supported by ONR and NSF.]

\section{1:55}

4pAO3. Acoustic measurements of boundary layer turbulence, sediment concentration and sediment flux using the Bistatic Coherent Doppler Velocity and Sediment Profiler (BCDVSP). Tim Stanton (Oceanogr. Dept., Code OC/St, Naval Postgrad. School, Monterey, CA 93943)

A pulse-to-pulse coherent acoustic doppler using a bistatic transducer configuration has been developed to study oceanic boundary layers. The instrument uses a central narrow-beam transceiving transducer, with three surrounding fan-beam response receiving transducers directed toward the narrow volume ensonified by the central transducer. Short acoustic pulses ensonify approximately 1 -cm-long sections of the water column below the 2-deg-width transmitter transducer, and acoustic energy reflected from scatterers in the water column are received by all four transducers. Coherent sampling methods provide high resolution doppler frequency measurements. The four doppler estimates allow three component velocity vectors to be estimated concurrently with the backscatter amplitude in the same small volumes over a $75-\mathrm{cm}$ range, at $1-\mathrm{cm}$ resolution and $20-\mathrm{Hz}$ sample rate. Laboratory calibration of the acoustic backscatter using in situ sand samples provides sediment concentration profiles over a 0.001 to $25 \mathrm{~g} / 1$ concentration range. The BCDVSP has been used in two ONR studies of the oceanic bottom boundary layer (BBL) on the inner shelf. Examples of BBL turbulent structure and sediment concentration will be shown from the SandyDuck near shore experiment in 1997, and from two inner shelf sites during the Shoaling Waves Experiment. 
4pAO4. Coherent Doppler profiler measurements of near-bed suspended sediment fluxes for different bed states. Carolyn E. Smyth, Alex E. Hay (Dalhousie Univ., Halifax, NS B3H 4J1, Canada), and Len Zedel (Memorial Univ., St. John's, NF)

Vertical profiles of vertical turbulence intensity and vertical sediment fluxes were collected by an acoustic coherent Doppler profiler at two locations: Queensland Beach (Nova Scotia), and Duck (North Carolina). Observations of the turbulence intensity over different bed states (irregular ripples, cross ripples, linear-transition ripples, and flat bed) reveal that the near-bed turbulence levels are strongly affected by bed forms. This study examines the mechanisms of distributing suspended sediments and generating near-bed turbulence for the four bed states based on the characteristics of two previously observed mechanisms: diffusion and vortex shedding. Wave-phase averages of turbulence intensity, suspended sediment concentration, and suspended sediment fluxes are compared to vortex shedding and diffusion signatures. Evidence of vortex shedding is found for the low-energy ripples, but no signatures of diffusion are observed. Two diffusion models including a bed stress model, and an eddy diffusion model are found to predict near-bed turbulence levels reasonably well, but predictions are inconsistent with the trend of the data. A vortex-shedding model [J. F. A. Sleath, J. Fluid Mech. 182, 369-409 (1987)] predicts the vertical structure of the turbulence for rippled beds when the ripple wavelength is used as a ripple roughness.

\section{2:35}

4pAO5. Analysis of acoustic measurements of suspended sediments collected in a large-scale wave flume facility. Peter D. Thorne (Proudman Oceanographic Lab., Bidston Observatory, Bidston, UK)

Our capability to accurately predict suspended sediment concentration in the marine environment is relatively limited; generally, measuring techniques to obtain suspended concentration have been somewhat primitive. In recent years acoustics has been applied to the measurement of suspended sediments, and through a series of theoretical and experimental studies, a comprehensive description of the interaction of sound with suspensions of marine sediments has been developed. In the present work these developments have been applied to obtain detailed acoustic profiles of suspended sediment concentration under waves in a large-scale flume facility. Utilizing the acoustic concentration profiles, an assessment of suspended sediment models for predicting sediment concentration has been conducted, and the underlying intrawave mechanisms of sediment entrainment investigated.

\section{2:50}

4pA06. Three-dimensional imaging of sand transport processes over wave ripples. Peter Traykovski, James D. Irish (Appl. Ocean Phys. and Eng. Dept., Woods Hole Oceanogr. Inst., Woods Hole, MA 02543), and Alex E. Hay (Dalhousie Univ., Halifax, NS B3H 4J1, Canada)

While the interactions between bedforms, suspended sediment transport and the forcing hydrodynamics are inherently three dimensional, most observations have been either point samples as a function of time, or 1-D vertical imaging systems. Recently, acoustic imaging systems have been designed to make 2-D maps of either bedform plan view topography (rotary fan beam sonars) or suspended sand concentration and bedform profiles (rotary pencil beam sonars). In the winter of 1999-2000, Woods Hole and Dalhousie investigators combined several of these 1- and 2-D systems to produce a data set on the 3-D structure of the suspended sand concentration field and its relation to the seafloor topography and forcing hydrodynamics. The data revealed that although the wave orbital scale ripples can be quite 2-D at times (i.e., long crested), the suspended sediment has considerable structure in the along-crest direction. The bedforms were observed to migrate in the onshore direction, and clouds of suspended sand were observed to be re-suspended in the lee of a ripple crest and then were advected back and forth over the crest.
$3: 20$

4pA07. Field observations of bedforms in high sediment transport conditions. Anna M. Crawford and Alex E. Hay (Dept. of Oceanogr., Dalhousie Univ., Halifax, NS B3H 4J1, Canada)

In the first reported use of MHz-frequency acoustics to study smallscale bedforms in the near shore zone, Dingler and Inman were able to observe the evolution of transition ripples under energetic groupy waves near the flat bed threshold. They found that these low-relief, long-crested bedforms appeared to be obliterated under the largest waves in a group, and subsequently reformed in one or two wave periods [J. Dingler and D. Inman, Proc. 15th Coastal Conf., Vol. II, 2109-2126 (1976)]. More recently, measurements of linear transition ripples were made during an autumn storm event using scanning wide beam and fixed narrow beam sonars, as well as a high resolution laser-illuminated video system. These observations also indicate the occasional apparent disappearance of these ripples, and also their reformation at the same locations. The question arises as to whether the disappearance is apparent (the result of increased near bed sediment concentration and the definition of bed elevation based on reflection amplitude) or real (planing off of the ripple crests by sheet flow).

\section{3:35}

4pA08. A three-dimensional coherent Doppler profiler. Len Zedel (Phys. and Physical Oceanogr. Dept., Memorial Univ., St. John's, NF A1B 3X7, Canada, zedel@physics.mun.ca) and Alex Hay (Dalhousie Univ., Halifax, NS B3H 4J1, Canada, hay@phys.ocean.dal.ca)

A 1.7-MHz coherent Doppler sonar system has been designed for near-shore environments that provides three-dimensional velocity profiles over an $\mathrm{O}(0.5-\mathrm{m})$ depth range. The use of a bistatic geometry allows three independent components of velocity to be measured simultaneously in time and coincident in space. The system was calibrated in a tow-tank facility for velocities up to $2 \mathrm{~m} / \mathrm{s}$. For multiple pulse-pair ensembleaveraged velocity estimates generated at a rate of 30 profiles $/ \mathrm{s}$ in $0.7-\mathrm{cm}-$ depth bins, vertical velocity accuracies of the order $1 \mathrm{~cm} / \mathrm{s}$ are achieved. For the horizontal components (based on bistatic measurements), standard deviation in velocity estimates of about $5 \%$ is realized with absolute accuracies of about $2 \%$. In the configuration as tested, flow disturbance around the instrument introduces a bias of about 5\%: this disturbance is modeled using potential flow past a cylinder. The disturbance errors could be corrected to the free-stream velocities using the present data set. Such a correction would not, however, reflect the actual water speed at the point of measurement. [This research was funded by the Office of Naval Research Coastal Sciences Program, and the Natural Sciences and Engineering Research Council of Canada.]

4pAO9. Effects of the top transition layer in marine sediments on seabed roughness and volume scattering. Anatoliy N. Ivakin (CNRS/ LMA/ASM2, 31 Chemin Joseph-Aiguier, 13402 Marseille Cedex 20, France; also affiliated with AOPE/WHOI, Woods Hole, MA 02543, anivakin@hotmail.com)

Most models of high-frequency seabed scattering normally assume that the sediment is a statistically homogeneous half space and there is a discontinuity of acoustical parameters at the water-sediment interface. It is known, however, that in many cases properties of the very top layer of real marine sediments actually represent almost continuous transition within a few centimeters from those in water to those essentially constant in sediments at greater depths. At the Key West site, e.g., a definite transition layer in silty sand was found to be of 6-cm thickness. The discontinuity at the water-sediment interface, if it exists, is actually much smaller than assumed in conventional models of high-frequency scattering, where roughness scatter component is usually predicted to be strong 
and dominating for most types of sediments except very fine ones, such as mud and fine silt. In this paper, effects of the transition layer on both roughness and volume-scattering components are considered. These effects are shown to reduce substantially the role of roughness scattering at high frequencies. In particular, the roughness contribution is shown to be strongly dependent on real discontinuity of acoustical parameters at the water-sediment interface, which can be significantly reduced even for some sands due to existence of the transition layer. The possibly dominating role of volume scattering caused by continuous and discrete heterogeneity of the sediment is discussed. The necessity of more detailed study of physical properties of the transition sediment layer is emphasized. Related issues of interpretation of recent results on seabed scattering obtained in the Key West and SAX99 experiments are discussed as well. [Work supported by ONR-US and CNRS-France.]

\title{
Session 4pMUa
}

\section{Musical Acoustics: Historical Brasses and Related Instruments}

\author{
R. Dean Ayers, Chair \\ Department of Physics and Astronomy, California State University, Long Beach, California 90840-3901
}

Chair's Introduction-1:25

Invited Papers

$1: 30$

4pMUa1. An overview of the brass collection in the Kenneth G. Fiske Museum. Albert Rice (Kenneth G. Fiske Museum of The Claremont College, Claremont, CA 91711, alrice@cuc.claremont.edu)

The Kenneth G. Fiske Museum of Musical Instruments at The Claremont Colleges is one of only seven comprehensive musical instrument museums in the United States. Its collection of brasses and cup-mouthpiece instruments made of wood consists of over 400 instruments dating from the 18th through the 20th centuries. It includes cornets, trumpets, bugles, trombones, French horns, mellophones, euphoniums, tenor horns, ophicleides, keyed bugles, over-the-shoulder horns, serpents, cornettos, Russian bassoons, bass horns, tubas, instruments from Africa, Tibet, China, and other countries. The collection is especially rich in its representation of important 19th century American makers and of examples of valve designs such as Stoelzel, Berlin Pumpen, Vienna, Allen, and various designs of the Perinet valve. Valves were initially introduced by German makers in 1818, and were gradually accepted by players during the 1830s and 1840s. The Perinet valve of 1838 emerged as the standard design only by the 1870 s and 1880 s. With the use of slides of several instruments the presentation will illustrate four broad aspects of the collection: (1) the development of the low brasses; (2) types of valves; (3) brasses in Asia and other countries; and (4) unusual designs.

2:00

4pMUa2. The evolution of lip-reed instruments and their manufacture. Robert W. Pyle, Jr. (11 Holworthy Pl., Cambridge, MA 02138)

Lip-reed instruments began in prehistory as animal horns and tusks and have evolved into the brass instruments of modern orchestras and bands. After a brief look at instruments of biblical times and earlier, this paper will concentrate on developments from the 15th century to the present. By the mid-18th century, the typical trumpet bell contour had changed from an "animal horn" shape to something very close to that of the present-day trumpet and players had achieved remarkable technical proficiency, as shown in the compositions of Bach, Handel, and others. On the other hand, manufacturing techniques had changed hardly at all and the instruments were still mechanically simple. The introduction of steam power in the late 18th century profoundly changed metal-working techniques and, with the invention of the valve, led to an explosion in the variety and complexity of brass instruments. Of the numerous dead branches and twigs on the brass family tree of the 19th century, some were killed by bad acoustics, some by economics, and some by poor marketing. In our own day, computer-controlled machining not only has changed traditional manufacturing economics, but also has allowed the production of new components that would formerly have been prohibitively expensive.

2:30

4pMUa3. An acoustical comparison of the serpent and the ophicleide. D. Murray Campbell (Dept. of Phys. and Astron., Univ. of Edinburgh, Edinburgh EH9 3JZ, UK)

The serpent is a lip-excited wind instrument with an air column length of around $2 \mathrm{~m}$ and an approximately conical bore. In the form in which it emerged in France in the sixteenth century, it is a wooden tube with six finger holes and a chromatic compass of three octaves. The serpent suffers from some problems related to the fact that all the holes are covered directly by the fingers. The holes are grouped in two clusters, and the hole diameter is too small to provide adequate venting. The ophicleide, invented in the early nineteenth century, has a similar bore profile to the serpent. Eleven or more side holes in the thin-walled metal tube are covered by lever-operated padded keys; these allow most notes to be obtained without cross fingering, and the size and placing of the holes are not limited by the anatomy of the human hand. Several acoustical investigation techniques, including input impedance analysis, pulse reflectometry and excitation by an artificial mouth, have been used to investigate the extent to which the ophicleide is a successful solution to the acoustical problems posed by the serpent. 
4pMUa4. A survey of the historical and acoustical development of the horn. Brian Holmes (Dept. of Phys., San Jose State Univ., San Jose, CA 95192-0106)

In the 17th century, the hunting horn entered the orchestra. With its shallow mouthpiece and rapidly flared bell, the baroque horn had a bright sound like that of the trumpet. Indeed, the same musicians often played either instrument. In modern authentic performances, players use side-vents or hand-stopping to improve intonation, though no evidence suggests that 18th century players used these techniques. When the hand entered the bell, the bell was widened and the mouthpiece deepened, giving the instrument its modern character. Despite the invention of the valve, acoustics requires that the hand remain in the bell. The modern double horn, with its wider bell and rotary valves, was introduced by Kruspe in 1902. Older designs (such as the Vienna horn, with its narrower bell, terminal crook, and curious Vienna valves) persist in some areas. The drive to compete with trumpets for volume and recordings for accuracy has led some modern players to adapt higher pitched descant horns.

\section{$3: 30$}

4pMUa5. Design characteristics of nineteenth century American-made brass instruments. Richard P. Birkemeier (Dept. of Music, California State Univ., Long Beach, 1250 Bellflower Blvd., Long Beach, CA 90840, birkemei@csulb.edu)

The brass band was arguably the most popular form of entertainment for most American citizens in the nineteenth century. This ensemble flourished in nearly every town and eventually led directly to the development of jazz, the basis for all American popular music in the twentieth century. American brass instrument design underwent significant changes in a relatively short period of time during the second half of the nineteenth century. These design changes resulted in noticeable variations in instrumental timbre, changes that clearly had an effect on the music written for wind bands during this period. This presentation will feature two complete sets of nineteenth century brass band instruments, one from the Civil War era and one from the end of the century. They will be analyzed from the following perspectives: design specifications of bore, bell shape, valve configuration and mouthpiece design; weight and thickness of metal; and instrument timbre as demonstrated in live performance. It is hoped that the comparison of design characteristics followed by the actual sounds of the instruments will offer insights into this most important of relationships for the musician.

\section{Contributed Papers}

\section{4:00}

4pMUa6. Pitch drift as a result of just intonation. Patrick K. Mullen (Art Dept., CSU Long Beach, Long Beach, CA 90840)

The most easily recognized difficulty with just tuning is the large and confusing array of pitches that must be made available on a keyboard instrument in order to allow modulation to several keys. A given note name may have more than one pitch associated with it, sometimes several, depending on the context in which it is used. Using computer-generated instruments that provide a limitless supply of unique pitches, just intervals can be used exclusively and explored thoroughly. When applied to Giovani Gabrieli's Sonata Pian' E Forte, the strict use of just tuning forces the pitch center to drift lower as the music progresses. When pitches are held across certain chord changes, lower versions of previous pitches must be used in order to maintain just tuning. Though most nonkeyboard acoustic instruments, the brasses in this case, can vary the tuning of each pitch somewhat, most cannot match the degree of drift that occurs in the Gabrieli ("A" begins the piece at $440 \mathrm{~Hz}$ and ends it at $\sim 398 \mathrm{~Hz}$ !). For players of these instruments a compromise between physical tuning limitations and the ideal of just intonation must be made. Players use just intervals wherever possible, until the pitch drift forces a decision as to where the "rub" will be. The pitch-drift phenomenon will be demonstrated.

\section{$4: 15$}

4pMUa7. Musical instruments of antiquity as illustrated in The Adventures of Asterix the Gaul. Daniel A. Russell (Sci. \& Math Dept., Kettering Univ., 1700 W. Third Ave., Flint, MI 48504)

The Adventures of Asterix the Gaul, a series of books written by Goscinny and illustrated by Uderzo, between 1960 and 1999, have received much acclaim for the attention to detail in their drawings of ancient architecture and civilization. Equally interesting to a musical acoustician are the illustrations of musical instruments (including carnyx, buccina, lur, bagpipe, harp, lyre, pipes, drums, and singing) used by ancient Romans, Greeks, Egyptians, and Gauls. This talk will compare Uderzo's illustrations to photographs of period instruments and comment on their acoustic qualities, performance techniques, and the roles they played in their respective societies, both in real history and as experienced by Asterix and his friends. 


\title{
Session 4pMUb
}

\section{Musical Acoustics: Concert Performance by the Americus Brass Band}

\author{
R. Dean Ayers, Chair \\ Department of Physics and Astronomy, California State University, Long Beach, California 90840-3901
}

Chair's Introduction-5:00

\begin{abstract}
A performance technical session featuring the Americus Brass Band will be held on Thursday from 5:00 to 6:00 p.m. in the California Ballroom. The band re-creates the historical town band of Americus, Georgia, which became the regimental band of the 4th Georgia Volunteers in the Confederate Army. The Americus Brass Band has toured nationally for eight years, appearing at major Civil War battle reenactments, balls, and other related "living-history" events. They have produced three critically acclaimed CDs and recorded sound-track music for and/or appeared on-screen in a dozen major motion pictures and television shows. Playing their antique instruments and dressed in period costume, the Americus Brass Band will offer a rousing concert of music from the Civil War to the Buffalo Bill Wild West Show, demonstrating how brass instruments and brass music evolved in the United States during the last half of the nineteenth century. Visit them at: http://www.csulb.edu/ birkemei/abbweb/abb.htm
\end{abstract}

THURSDAY AFTERNOON, 7 DECEMBER 2000 CATAMARAN/TRIMARAN ROOMS, 1:30 TO 4:55 P.M. Session 4pNS

\section{Noise: Hearing Protection Standards}

\author{
Elliott H. Berger, Chair \\ E-A-R, 7911 Zionsville Road, Indianapolis, Indiana 46268-1657 \\ Chair's Introduction-1:30 \\ Invited Papers
}

$1: 35$

4pNS1. New ISO standards for hearing protectors. Torben Poulsen (Dept. of Acoust. Technol., Bldg. 352, DTU, DK-2800 Lyngby, Denmark, tp@dat.dtu.dk)

Working Group 17 under ISO/TC43/SC1 deals with measurement of hearing protector attenuation. WG17 is presently involved in revision of the present 4869-1 standard (especially sound field requirements and reproducibility estimations) and formulation of a new standard where inexperienced hearing protector users are mandatory as test subjects. The latter procedure has recently been introduced in the USA, Australia, and New Zealand. Both the present and the new standard use the Real Ear At Threshold (REAT) method. Results from investigations of the sound field in different test environments will be presented and the status of coming standards for impulse noise and for devices with active noise control will be mentioned.

\section{$1: 55$}

4pNS2. Ongoing experience with laboratory-based subject-fit REAT methods for measuring hearing protector attenuation. Elliott H. Berger and Ronald W. Kieper (E-A-R, 7911 Zionsville Rd., Indianapolis, IN 46268-1657)

As data have accumulated indicating the difficulty in basing estimates of field-delivered (i.e., real-world) protection for groups of users upon classical laboratory-derived optimum attenuation data, more attention has been directed towards standards intended to provide better simulations of the real world. The development of ANSI S12.6-1997 Methods for Measuring the Real-Ear Attenuation of Hearing Protectors, is one approach towards addressing this problem. The standard includes both experimenter-supervised fit and subject-fit methods. The latter method, designated as Method B, requires the use of audiometrically experienced subjects who are naive in the use of hearing protection. This method is intended to approximate the upper limits to the attenuation that can be expected for groups of occupational users. It yields mean attenuation values, more so for earplugs than earmuffs, that are substantially lower 
and standard deviations values that are higher than previously found using ANSI standards. This paper will present the latest Method-B test data, review ongoing studies designed to further evaluate the Method-B protocol, and will comment on efforts to more widely disseminate Method-B data and the associated noise reduction rating (Subject FIT) [NRR(SF)] which can be derived from such values.

\section{2:15}

4pNS3. Measurement and classification of hearing protectors in New Zealand and Australia. George Bellhouse (Bel Acoustics Consulting, P.O. Box 22 073, Khandallah, Wellington, New Zealand, george.bellhouse@xtra.co.nz)

An overview of the method of the measurement of the attenuation of hearing protectors using the subject-fit protocol according to the Joint Australian/New Zealand Standard AS/NZS 1270-1999: "Acoustics-Hearing Protectors" is presented including a comparison with ANSI S12.6-1997: "Methods of Measuring the Real-Ear Attenuation of Hearing Protectors"; Method "B": Subject Fit. In this Australian/New Zealand Joint Standard, hearing protectors are rated in terms of a "class" rather than given an attenuation value. The system of classifying hearing protectors is detailed including the actual method of the determination of the "class" of a hearing protector. The use of the Australia/New Zealand "class" system in the workplace is illustrated by way of an example. This describes the use of LAeq,T, the determination of LAeq, $8 \mathrm{~h}$ and how these parameters relate to the "class" of a hearing protector. The benefits and drawbacks of the system used are compared with other rating systems.

\section{$2: 35$}

4pNS4. Now what do we do with these good numbers? John R. Franks and William J. Murphy (Hearing Loss Prevention Section, NIOSH, M.S. C-27, 4676 Columbia Pkwy., Cincinnati, OH 45226-1998)

Present ANSI and ISO standards suggest calculating the mean real-ear-attenuation at threshold (REAT) and the standard deviations as well. These are used for calculating assume protection values (APVs) that are incorporated in calculations of the NRR in the United States and the SNR and HML in Europe and elsewhere. One of the discoveries from intense analysis of both experiment-fit and subject-fit REAT values is that they are not always unimodally and normally distributed around a mean or median, thus weakening the statistical relevance of the mean and standard deviation of the REATs. Methods for determining APVs that take into account the distribution of REATs and yet allow the computations to be otherwise unaffected will be presented. Additionally, suggestions for ratings other than the NRR, SNR, and HML will be made.

\section{2:55}

4pNS5. Measuring the noise attenuation of shotblasting helmets. Jacqueline A. Patel (Noise and Vib. Section, Health and Safety Lab., Harpur Hill, Buxton, Derbyshire SK17 9JN, UK, jacqueline.patel@ hsl.gov.uk)

European Standard EN 271: 1995 deals with the construction of air-fed shotblasting helmets. It specifies minimum requirements for face, eye, and respiratory protection and tests the noise generated by the breathing air supply. However, it does not test the helmet's ability to attenuate the very high levels of noise generated by the shotblasting process. This is a major omission. A test method was developed for measuring the noise attenuation provided by shotblasting helmets against typical shotblasting noise. It involved objective measurements using the head and torso simulator (HATS) currently prescribed by EN 271, fitted with miniature microphones. Using such a HATS was shown to give attenuation values that correlate well with those measured using human subjects. Results from this project showed: the HATS already used by manufacturers to show compliance of their product with EN 271 can also be used to provide information on the helmet's noise attenuation; and the same HATS can be used, in place of human subjects, to measure the air supply noise according to the method defined in EN 271. The findings from this work will be used to try to influence future revisions of EN 271 so that the noise attenuation of shotblasting helmets is considered.

\section{3:15-3:30 Break}

\section{$3: 30$}

4pNS6. Effect of active noise reduction (ANR) and passive hearing protectors on detectability of a vehicle backup alarm in noise. John G. Casali (Auditory Systems Lab., Virginia Tech, Blacksburg, VA 24060, jcasali@vt.edu), Erika Dabney (Xerox Corp., Rochester, NY), Gary S. Robinson (Virginia Tech, Blacksburg, VA 24060), and Dan Gauger (Bose Corp., The Mountain, MA)

In a within-subject laboratory experiment, ten subjects of normal-hearing abilities listened for a standard vehicle backup (reverse) alarm that was presented against either an 85 or $100 \mathrm{dBA}$ background noise having either a pink or red spectrum. Subjects' masked thresholds to the alarms were determined under four listening conditions: ANR earmuff, passive earmuff, foam earplug, and unprotected (at $85 \mathrm{dBA}$ only). To become immersed in a workload situation, subjects continuously attended to a computer-generated visual monitoring task while performing the auditory detection experiment. Analysis of variance and post-hoc tests were applied to the mean masked threshold results. In $85 \mathrm{dBA}$ noise, masked thresholds were significantly lower when any protector was worn, as compared to the unoccluded condition, lending support for the use of hearing protection with normal hearers when backup alarm signals need to be heard in noise. The ANR earmuff provided a significant advantage over the passive earmuff in both levels of the red noise, and for both noise spectra at $100 \mathrm{dBA}$. However, the foam earplug yielded lower masked thresholds than either other protector in $100 \mathrm{dBA}$ noise. Subjects' perceptions about efficacy of warning signal detection and about comfort revealed no differences among the three protectors. 
4pNS7. Hearing protector performance for impulse noise. Daniel L. Johnson (Brüel Bertrand \& Johnson Acoustics, 4719 Mile High Dr., Provo, UT 84604)

Using Army volunteers, a series of studies were performed at the Blast Overpressure Test Site in New Mexico to establish safe exposure levels for impulse noise. The results of these studies demonstrated that for properly worn hearing protection, the limit of human exposure is set by the threshold of nonauditory damage. This nonauditory threshold can be depicted by a simple formula based on peak pressure, $A$-duration, and number of impulses. Using this information, a standard for impulse noise will be proposed in which the attenuation of the hearing protective need not be measured or predicted. The protector need only to be shown to protect adequately. One method for evaluating this adequacy is based on a procedure that verifies that Temporary Threshold Shifts of Hearing are not occurring. Another method is to measure the total A-weighted energy under the protection device or devices. The standard will also include a computer model that will provide the means for the user to predict the risk of hearing injury by analyzing the waveform of the impulse.

\section{Contributed Papers}

\section{4:10}

4pNS8. A statistical classifier for hearing protector REAT data. William J. Murphy and John R. Franks (Hearing Loss Prevention Section, NIOSH, M.S., C-27, 4676 Columbia Pkwy., Cincinnati, OH 45226-1998)

Hearing protector attenuation data have been demonstrated to exhibit bimodal characteristics due to poorly fitting protectors. The data have been modeled with two distributions [Murphy and Franks, J. Acoust. Soc. Am. 106, 2262 (1999)]. The bimodal distribution model was used as the basis of a statistical classifier for an individual's attenuation data to identify whether or not the data come from a poor or well fit distribution. Training classes were developed from ANSI S12.6 Method B subject fit data [Franks et al., Ear Hear. 21, 218-226 (2000)] for the EAR Classic ( $n$ $=25)$, EAR Express $(n=20)$ and Howard Leight MAX $(n=25)$ earplugs. Real ear attenuation at threshold was measured under circumaural headphones for each earplug with a panel of 20 subjects. Preliminary results suggest that data from 500 or $1000 \mathrm{~Hz}$ yield the best ability to separate the attenuations.

\section{$4: 25$}

4pNS9. The measurement of impulse noise protection by earmuffs with the use of various sound sources. Jan Zera (Central Inst. for Labour Protection, ul. Czerniakowska 16, 00-701 Warsaw, Poland)

Impulse attenuation of 23 hearing protectors from various manufacturers was determined by the transmission-loss method. Impulses were generated by a foil blaster, a starter gun, or by hitting a steel plate with a hammer. Peak levels of the impulses ranged from 130 to $160 \mathrm{~dB}$. The measurements were carried out using an artificial test fixture (ISO/TR 4869-3:1989), and a KEMAR manikin. Peak sound pressure level of acoustic impulses was measured with two microphones, one of which was placed under the earmuff and the other one outside the earmuff. The best agreement between impulse attenuation and the protectors' M, L, and SNR parameters was found when the foil blaster was used as a sound source. The results also show that the attenuation measured for impulses is usually smaller than the H, M, L, and SNR values given in the product data specification. [Work supported by the Polish Committee for Scientific Research Grant No. SPR 03.7.21.]

\section{$4: 40$}

4pNS10. Noise attenuation of an insertion-type hearing protector by mathematical model and experimental verification. Samir N. Y. Gerges, Elizabete Y. N. Bavastri (Federal Univ. of Santa Catarina (UFSC), Mech. Eng. (EMC-LVA), Trindade, Florianopolis, SC, Brazil, CEP: 88040-9000, gerges@mbox1.ufsc.br), and Roberto A. Tenenbaum (COPPE, Univ. Federal de Rio de Janeiro, Rio de Janeiro, Brazil)

The experimental real ear attenuation at threshold (REAT) method is based on the standards ANSI S12.6-1997 and ISO 4869-1990 which consider measurements on qualified subjects, in a qualified acoustic chamber. The REAT method is very expensive, time consuming, and has a very poor frequency resolution. Mathematical and numerical models, such as the one-dimensional plan wave model, finite-element method, and boundary-element method offer cheap, quick, and high-resolution results, especially for comparison and product development. In this paper, the outer ear canal is considered as a straight tube with an open end and the other end closed with an eardrum membrane of human acoustic impedance. For the open ear canal case (without hearing protector on), plan acoustic waves are considered as incident and reflected waves. In the case of a blocked ear (with the insertion hearing protector on), a model of three mediums is considered; air hearing protector air. In this model only one longitudinal wave propagation is considered. Material density and speed of sound are the only parameters considered. The noise insertion loss is calculated as the difference of the sound pressure level at the eardrum without and with the protector on. The results are compared with controlled experimental measurements. 


\title{
Session 4pPA
}

\section{Physical Acoustics, Noise, Standards Committee S2, Signal Processing in Acoustics and Structural Acoustics and Vibration: Novel Optical Techniques for Measuring Surface Vibration}

\author{
James M. Sabatier, Cochair \\ National Center for Physical Acoustics, University of Mississippi, Coliseum Drive, University, Mississippi 38677 \\ Benjamin A. Bard, Cochair \\ 19 Benjamin Road, Belmont, Massachusetts 02478 \\ Chair's Introduction-1:25 \\ Invited Papers
}

$1: 30$

\begin{abstract}
4pPA1. Whole field vibration measurements with laser-based techniques. Amit K. Lal and Cecil F. Hess (MetroLaser, 18010 Skypark Circle, Ste. 100, Irvine, CA 92614)

This paper discusses two techniques, a time-domain technique and a space-domain technique, to remotely measure 2-D vibration characteristics of a surface. The first technique is based on using multiple diode laser vibrometers to simultaneously measure the surface vibration at multiple points. The principles of laser Doppler vibrometry are briefly discussed and various architectures of systems to perform landmine detection are presented and discussed. Surface velocity data corresponding to various types of soils shaken by a buried geophone are presented. The second technique is based on laser speckle interferometry using a single digital camera to instantaneously capture quantitative phase information. The phase information of two consecutive frames is then converted to velocity profiles of the vibrating surface. The principles of a new method to perform instantaneous phase shifting interferometry are presented and modal analysis data of turbine blades obtained with an all-digital optical system are discussed.
\end{abstract}

\section{2:00}

4pPA2. Vibration analysis by digital shearography. W. Steinchen, G. Kupfer, and P. Mäckel (FB 15, Dept. of Mech. Eng., Lab. of Photoelasticity, Holography and Shearography, Univ. of Kassel, D-34109 Kassel, Germany)

Digital shearography, a laser interferometric technique in conjunction with the digital image processing, has the potential for vibration analysis due to its simple optical system and sensitivity against small rigid body motions. This paper will focus on its recent developments for vibration analysis and for nondestructive testing (NDT) by dynamic (harmonic) excitation. With the introduction of real-time observation using an automatically and permanently refreshing reference frame, both small and large rigid body motions are greatly suppressed. The development of a smaller and more mobile measuring device in conjunction with a user-guided comfortable program SHEARWIN enables the digital shearography to be applied easily as an industrial on-line testing tool.

\section{2:30-2:45 Break}

\section{Contributed Papers}

\section{2:45}

4pPA3. Laser-ultrasonic measurements of thin-film properties using surface acoustic waves. Donna C. Hurley (Natl. Inst. of Standards \& Technol., Boulder, CO 80305, hurley@boulder.nist.gov ) and Adrian J. Richards (CSIRO Telecommunications \& Industrial Physics, Lindfield, NSW 2070 Australia)

High-frequency, laser-ultrasonic methods to evaluate the mechanical properties of thin films have been developed. The approach is based on the optical generation and detection of surface acoustic waves (SAWs) to determine the frequency dependence of the phase velocity (dispersion relation). Broadband or variable-frequency, quasi-plane wave SAWs are generated by a line-focused, 200-ps pulsed laser. The out-of-plane SAW displacement amplitudes are measured using a path-stablized Michelson interferometer with line-focus detection. The apparatus incorporates differential photodiode detection with a $-3-\mathrm{dB}$ bandwidth of $40 \mathrm{kHz}-800$ MHz. With this system, several displacement waveforms over a range of propagation distances are acquired and used to determine the SAW dispersion. Dispersion relations up to $200 \mathrm{MHz}$ are presented for a variety of specimens, including a series of $\mathrm{Si}$ wafers with TiN films $0.2-1.3 \mathrm{mi}-$ crometers thick and containing residual stress. The results illustrate the applicability of this technique to various conditions such as stiffening and loading films and isotropic and anisotropic substrates. The experimental dispersion curves obtained with this method are compared to theoretical predictions to evaluate the film elastic properties.

\section{3:00}

4pPA4. Optical matched-filter and phased-array detection of surface acoustic waves. Todd Murray and Sridhar Krishnaswamy (Ctr. for Quality Eng., Northwestern Univ., Evanston, IL 60208-3020)

A method to optically measure surface acoustic displacements simultaneously over an array of detection points is described. Optical phase gratings are used to create a detection array of laser beams that is directed to the specimen. The scattered beams from the detection array are then collected and combined with a single reference beam in a photorefractive crystal in a multiplexed two-wave mixing configuration. The output beams of the resulting multiplexed photorefractive interferometer are imaged onto either a single photodetector (matched-filter scheme) or to separate 
photodetector elements (phased-array scheme). The optical matched filter requires less electronic overhead. By using different object beam footprints, it is possible to configure optical matched-filter receivers that selectively detect SAWs (broadband, narrow-band, chirped, etc.) with enhanced sensitivity. The responses of equispaced and chirped array receivers to propagating SAWs are discussed. The more general phasedarray configuration, although requiring greater electronic overhead, provides time-domain signals from each element for postprocessing. This system can therefore be synthetically focused by applying appropriate phase shifts between the detected signals to create images of the acoustic scattering region. Both these multiplexed optical detection schemes, with their high spatial resolution and subnanometer displacement sensitivities, have applications in nondestructive testing and materials characterization.

\section{3:15}

4pPA5. Measurements of wineglass resonance using a fiber-optic probe. Andrew A. Piacsek (Dept. of Phys., Central Washington Univ., 400 E. 8th Ave., Ellensburg, WA 98926)

A fiber-optic probe (MTI-2000 Fotonic Sensor) is used by Central Washington University physics students to measure the peak displacement of such vibrating surfaces as acoustically driven wineglasses, parametrically shaken liquids, and soap films. This presentation will consist of a brief description of the fiber-optic measurement system, a discussion of its advantages and limitations, and sampling of some recent data obtained by students.

\section{$3: 30$}

4pPA6. Phase-amplitude-modulated laser and microwave vibrometers. Dimitri Donskoy, Nikolay Sedunov, and Edward Whittaker (Stevens Inst. of Technol., Hoboken, NJ 07030)

The paper presents results of a new development of remote vibration sensors using modulated electromagnetic (optical and microwave) signals. The microwave sensor (vibrometer) utilizes the effect of phase modulation of a cw microwave carrier reflected from vibrating surfaces or subsurfaces. The optical vibrometer uses a similar principle of phase modulation of the microwave frequency modulating envelope of the optical carrier. Both optical and microwave vibrometers can share the same signal, generating and receiving electronics and algorithms. Using different carriers but shared electronics, both sensors can be combined in one device offering versatile capabilities for various applications. The developed concept has been experimentally validated for both optical and microwave sensors. The $2.5-\mathrm{GHz}$ portable vibrometer has been designed and tested. The de- scription of the vibrometers, examples of their applications, and comparison with commercial LDV systems will be discussed in the presentation.

\section{$3: 45$}

4pPA7. Measurement of grain size using diffused ultrasound energy. Guy Lamouche, Martin Lord, and Andr Moreau (Industrial Mater. Inst.NRC, 75 de Mortagne, Boucherville, QC J4B 6Y4, Canada, guy.lamouche@nrc.ca)

A new method is proposed to determine grain size in steel sheets by combining a laser-ultrasound technique with ultrasound energy diffusion. A short laser pulse impinging on a metal surface generates an ultrasonic wave packet that propagates through the material. This wave packet is scattered by the discontinuities it encounters at the boundaries of the metallic grains. At the beginning, this energy is quite localized near the generation point and then spreads in the sheet in a way that can be described as a two-dimensional diffusion process. This diffusion process can be followed by measuring the ultrasound field amplitude at the surface of the sheet at various distances from the generation point. This observation is performed remotely by using a laser light that is frequency shifted by the surface movement (Doppler effect). Theoretical considerations suggest that there should exist a relationship between the size of the grains responsible for the ultrasound scattering and the diffusion coefficient describing the ultrasound energy diffusion process. We present preliminary experimental results showing that such a relationship does exist, thus providing a method to remotely measure grain size in steel sheets.

\section{4:00}

4pPA8. The modes and loss mechanisms of a high $Q$ mechanical oscillator. Xiao Liu, J. F. Vignola (SFA, Inc., Largo, MD 20774), S. F. Morse, D. M. Photiadis, A. Sarkissian, M. H. Marcus, and B. H. Houston (Naval Res. Lab., Washington, DC 20375)

In order to understand the unusually high $Q$ nature of the double paddle oscillator employed in a number of recent experiments, laser Doppler vibrometry (LDV) measurements of the normal modes of the oscillator were carried out. The observed resonance frequencies and mode shapes are in excellent agreement with three-dimensional finite element simulations. The second antisymmetric torsional mode was found to have exceptionally good vibration isolation because of its mode shape. This explains its extremely small low temperature $(T<10 \mathrm{~K})$ internal friction (2 $\times 10^{-8}$ ). A design criterion is established to minimize the damping of similar oscillators by correlating features of the measured and predicted mode shapes. 


\title{
Session 4pSA
}

\section{Structural Acoustics and Vibration: Sound/Structure Interaction}

\author{
Karl N. Grosh, Chair \\ Department of Mechanical Engineering and Applied Mechanics, University of Michigan, 2350 Hayward Street,
} Ann Arbor, Michigan 48109-2125

\section{Contributed Papers}

\section{$1: 30$}

4pSA1. Computational evaluation of satellite equipment panel modal densities and radiation efficiencies. Stephen C. Conlon (Hughes Space and Communications Co., Bldg. S24 MS D563, P.O. Box 92919, Los Angeles, CA 90009) and Stephen A. Hambric (Appl. Res. Lab., Penn State Univ., State College, PA 16804)

During the design phases of communications satellites Statistical Energy Analysis (SEA) methods are often used to predict critical equipment panel structural-acoustic vibration responses. Difficulties arise in the SEA modeling due to the nonhomogeneous nature of these complex equipment panels. Panel constructions are typically sandwich honeycomb (thick) cored panels with internal embedded heat pipes, external attached stiffeners, external thermal doubler plates, as well as the attached equipment itself. The resulting built up structure is very difficult to analyze using SEA theoretical asymptotic values. This work computationally evaluates the modal density and radiation efficiency of a typical communications satellite equipment panel utilizing a detailed finite-element model along with a lumped parameter radiation representation developed by Fahnline (1997). The full built up nonhomogeneous panel, as well as the uniform baseline panel, are evaluated using both idealized and realistic boundary conditions. The characteristics of the nonhomogeneous equipment panels are shown to vary significantly from commonly used SEA values. Simple curve fits are developed for future SEA evaluations of similar structures.

\section{$1: 45$}

4pSA2. Far-field radiation from a source in a flat rigid baffle of finite size. Joerg W. Panzer (New Transducers Ltd., Huntingdon PE29 6FW, UK, j.panzer@nxtsound.com)

The far-field radiation of a source in a flat rigid baffle of finite size is derived. The velocity distribution of the source is arbitrary as well as the shape of the source and the baffle. The derivation covers the two-sided as well as the single-sided radiating case. The approach makes use of the Kirchhoff-Helmholtz equation as well as of spatial Fourier transforms of the source and the baffle shape.

\section{2:00}

4pSA3. An analytical solution for the self and mutual radiation resistances of a rectangular plate. W. L. Li (United Technologies Carrier Corp., A+R Bldg., Carrier Pkwy., Syracuse, NY 13221)

It is widely accepted that, for an arbitrary acoustic wave number, the radiation resistance of a simply supported rectangular plate has to be calculated through numerical integration. In this study, an analytical solution for the self and mutual radiation resistances is obtained in the form of the power series of the nondimensional acoustic wave number. Unlike the previous analytical or asymptotic solutions, it is not subject to any of the restrictions usually imposed upon the acoustic wave number. A few numerical examples are given simply to verify the solution and elucidate the reliance of the self and mutual radiation resistances on the acoustic wave number and the plate aspect ratio. It is shown that the present formulae are extremely efficient by cutting the CPU time by orders of magnitude in comparison with the traditional numerical integration scheme. This investigation successfully fills the long-existing gap in solutions for the moderate wave numbers that are often of primary concerns in an acoustic analysis.

2:15

4pSA4. Introduction of complex layer analysis (CLA) for acoustic radiation problems. David Feit and Daniel T. DiPerna (CDNSWC, 9500 MacArthur Blvd., W. Bethesda, MD 20817, feitd@nswccd.navy.mil)

The pressure radiated by a planar vibrating surface with a prescribed harmonic time-varying surface velocity can be represented as the inverse Fourier transform of the product of the velocity transform and the spectral form of the acoustic surface impedance. This integral representation usually cannot be evaluated in closed form, but can, however, be evaluated asymptotically in the far field, i.e., at field points large compared to the acoustic wavelength. In this note a novel approach, "complex layer analysis" (CLA) is introduced which involves an approximation of the acoustic impedance in terms of a rational function. Contour integration is then used to obtain an explicit expression for the near-field pressure distribution as a sum of waves propagating with wave numbers corresponding to the poles of the approximate acoustic impedance. Such an expression makes explicit the notion of pressure-field generation localizing to points of discontinuity in the velocity distribution. [Work supported by the Carderock Division Naval Surface Warfare Center ILIR Program.]

2:30

4pSA5. Determination of design trends in the structural acoustic optimization of a multi-ply laminated composite cylindrical shell. W. M. Johnson and K. A. Cunefare (School of Mech. Eng., Georgia Tech, Atlanta, GA 30332-0405)

Structural acoustic optimization (SAO) is a multidisciplinary design technique used to determine the optimal acoustic properties of a structural design. Previous works have applied structural acoustic optimization to stiffened and unstiffened aluminum cylindrical shells subject to acoustic and structural vibration excitation. Most recently, SAO has also been applied to multiply laminated composite cylinders. In this case the ply angles served as the design variables but were not allowed to vary over the cylinder length. In this work we perform a structural acoustic optimization on a laminated composite cylinder with variable ply angles along the cylinder length. This is accomplished with a computational design tool incorporating finite- and boundary-element methods. It is anticipated that these results will help to clarify what design trends control the interior acoustic environment. [This work was supported by the Structural Acoustics Branch of NASA Langley Research Center and Graduate Student Research Program Grant (NGT-1-52167).] 
4pSA6. Predictive approaches and measurements of far-field radiation from submerged partially coated (compliant layer) cylindrical shells. Peter C. Herdic, Brian H. Houston, Douglas M. Photiadis, and Earl G. Williams (Naval Res. Lab., 4555 Overlook Ave., Washington, DC 20375, herdic@code7136.nrl.navy.mil)

In-water laboratory experiments involving spatially dense near-field and far-field responses ( $k a=1$ to 20$)$ are reported for a number of point driven cylindrical shells with varying amounts of compliant material attached to the exterior. The first framed shell has a square opening in the otherwise fully coated surface. Two modeling approaches are employed to predict the far-field radiation from this opening. The first method is based on the Helmholtz Integral Equation (HIE), using a simple baffled plate model where only surface quantities around the opening are projected. A square piston in a cylindrical baffle is also implemented as a second predictive approach. The predictions are found to be in general agreement with the actual radiated values determined through holographic projections to recover the off-board quantities. The discussion includes the conditions necessary for an accurate model. Additionally, two shells with different levels of interior structural complexity are each investigated using nearfield acoustic holography (NAH) where the degree of compliant material coverage is varied. The physics of these systems will be discussed, including the nature of the normal modes, the energetics, and the degree to which interesting phenomena such as "tunneling" are observed.

\section{3:00-3:15 Break}

\section{3:15}

4pSA7. Numerical and experimental characterization of the transmission loss of complex composite panels. Maxime Bolduc, Raymond Panneton, Noureddine Atalla, and Jean-Luc Wojtowicki (GAUS, Mech. Eng. Dept., Univ. de Sherbrooke, 2500 boul de l'universit, Sherbrooke, QC J1K 2R1, Canada)

The noise reduction in aircrafts is one of the top priorities for the aerospace industry. In this paper, a thorough investigation is done on the reliability of numerical FEM/BEM methods to predict the sound transmission loss of aircraft-type panels. The investigation is based on the comparison between numerical predictions, using commercial and in-house software, and measurements for different configurations of panels: single plate, double plate with air cavity, and double plate with cavity absorption. Clamped boundary conditions are used for both the simulation and experiments. The porous material is modeled following Biot theory and the diffuse sound field is simulated as a superposition of plane waves at various directions. For the experimental part, the transmission loss is obtained by setting the panel between a reverberant and an anechoic room. The transmitted power is measured with an intensity probe allowing fine characterization of the effects of complexities such as the boundary conditions and leaks. Furthermore, the quadratic velocity is measured as a means of validation for the mode shapes. Discussions on the numerical procedure to follow for providing reliable transmission loss predictions and a discussion on the discrepancies between the experiments and the numerical simulations will be done.

\section{3:30}

4pSA8. Transmission loss of heterogeneous porous materials. Noureddine Atalla (GAUS, Dept. of Mech. Eng., Univ. de Sherbrooke, QC, Canada) and Franck Sgard (LASH, DGCB URA CNRS 1652, ENTPE, 69518 Vaulx-en-Velin Cedex, France)

Recently, the authors have investigated the acoustic absorption performance of heterogeneous materials made up from a combination of thin porous patches, with different acoustic properties. It has been shown that absorption could be significantly increased in the case of properly designed macroperforated wools in a large frequency band. This paper investigates the transmission loss of similar macroheterogeneous materials. The studied materials are made up of porous elastic materials containing solid inclusions. They are placed into an infinite rectangular waveguide.
The numerical model used combines a finite-element formulation for the heterogeneous porous material and a modal description of the acoustic field in the waveguide. The coupling between the solid and the porous patches is accounted for naturally through the use of a modified Biot's formulation. Numerical results are presented to show the effect of several geometric and physical parameters on the transmission loss performance of heterogeneous porous materials.

\section{$3: 45$}

4pSA9. A new finite/infinite scheme to model acoustic trim under realistic working conditions. Jean-Pierre Coyette, Gregory Lielens, and Jean-Louis Migeot (Free Field Technologies, 16 Pl. de l'Universit, B-1348 Louvain-la-Neuve, Belgium)

The paper presents a finite-element scheme modeling all aspects of the behavior of acoustic trim: insulation, absorption, and damping. The models can combine different materials (viscoelastic, poroelastic, incompressible), acoustic fluid, and include complex effects like visco-thermal dissipation in thin air layers. Unbounded acoustic regions are modeled using stabilized conjugated infinite elements that present exceptional convergence properties. The structure can be subjected to a number of different excitations: kinematical (fixed motion), dynamical (discrete or distributed forces) or acoustical. An incident diffuse sound field or distributed pressure fluctuations corresponding to aerodynamic boundary layers can also excite the structure. The presentation will be organized as follows: (1) a brief review of the techniques involved: finite-element method for the structure and the acoustic near field, infinite elements for the acoustic far field; (2) material models considered: viscoelastic, poroelastic, incompressible, acoustic (including viscothermal effects); (3) excitation models: kinematical, dynamical, acoustical, aerodynamic; (4) validation of the approach; (5) three application cases: comparison of the dynamic behavior of a treated and untreated shell, acoustic transparency of an insulating sandwich, evaluation of the acoustic impedance of a porous layer.

\section{4:00}

4pSA10. Dynamic structural-acoustic-piezoelectric finite-element analysis of a sonar array for a supercavitating high-speed underwater vehicle. Robert M. Koch (Adv. Technol. Div. Naval Undersea Warfare Ctr., 1176 Howell St., Newport, RI 02841-1708, kochrm@npt.nuwc.navy.mil)

Three-dimensional nonlinear dynamic finite-element analysis (FEA) is utilized herein in order to examine the coupled structural, acoustic, and piezoelectric noise propagation physics within a high-frequency sonar array for a supercavitating underwater research vehicle. Specifically, steadystate dynamic simulations of the sonar array's coupled performance are carried out that model both (a) the acoustic radiation field resulting from electrically actuated ceramic array elements, and (b) the ceramic array element electrical response due to incident structure-borne excitation; fluid-borne excitation is being examined presently and may also be presented if completed in time. This paper focuses primarily on the interesting issues related to accurately modeling such a complex coupled system. For example, the frequency-dependent properties of a nonlinear elastomer absorption material within the array must be carefully characterized at very high frequencies in order to gain accurate results. Additionally, determining electrical properties of the piezoelectric ceramic material can pose a significant problem. Finally, performing a three-dimensional simulation at high frequencies with coupled physics analyses is not a trivial undertaking.. Laboratory shaker experiments performed in order to validate the modeling will also be presented.

\section{$4: 15$}

4pSA11. Effects of tube shape on nonlinear resonant oscillations of interior gas. Young-Doo Chun and Yang-Hann Kim (NOVIC, Dept. of Mech. Eng., KAIST, Science Town, Taejon 305-701, Korea, yhkim@mail.kaist.ac.kr)

An investigation is conducted to get an outline of designing the tube shape for a sonic compressor with high efficiency. When a closed tube oscillates with its resonant frequency, the interior acoustic properties such as density, velocity, and pressure undergo very large perturbation with 
nonlinear patterns. In order to analyze the nonlinear acoustic phenomena such as harmonic generation, shock formation, and resonant frequency shift, two-dimensional axisymmetric governing equations are derived and solved numerically. Numerical simulations are performed to investigate the effect of the tube shape that gives us maximum possible pressure.

Results show that the resonant frequency and patterns of pressure waves strongly depend on not only the tube shape but also the amplitude of driving acceleration. The degree of nonlinearity of pressure signals is measured by the newly defined nonlinear energy ratio of the signals. The nonlinear energy ratio well describes not only the energy transfer to higher harmonics but also the compression efficiency, so that it can be a good measure for designing tube shape.

THURSDAY AFTERNOON, 7 DECEMBER 2000

PACIFIC SALONS A, B AND C, 1:25 TO 5:15 P.M.

\title{
Session $4 \mathrm{pSC}$
}

\section{Speech Communication: Kenneth Stevens' Contributions to Speech Research: Influences and Future Directions}

\author{
Joseph S. Perkell, Cochair \\ Massachusetts Institute of Technology, Room 36-591, 50 Vassar Street, Cambridge, Massachusetts 02139-4307 \\ Stefanie R. Shattuck-Hufnagel, Cochair \\ Speech Communication Group Research Laboratories, Massachusetts Institute of Technology, 77 Massachusetts Avenue, \\ Cambridge, Massachusetts 02139
}

Chair's Introduction-1:25

Invited Papers

$1: 30$

4pSC1. Ken Stevens and cross-linguistic phonetic studies. Peter Ladefoged (Phonet. Lab., Linguist. Dept., UCLA, Los Angeles, CA 90095-1543, oldfogey@ucla.edu)

Many years ago we realized that one person's pathological voice quality might be a necessary part of another person's phonological contrast. Some American English speakers may have a very breathy voice, but a Gujarati speaker may need that voice quality to distinguish the word meaning "outside" from the word meaning "twelve." It was largely Ken Stevens's work, in personal communications, that enabled us to quantify these distinctions. This led to the notion that it was possible to describe a continuum of phonation types: voiceless, breathy, voiced, creaky, glottal closure. Using a modified version of another notion suggested by Stevens, we were able to consider possible quantal states along the parameter of glottal stricture. Further investigation of different languages suggested that contrasts along a single parameter may not be as straightforward as they seemed, and a number of "helping features" (yet another Stevens notion) were often involved. Despite being misled by the binary notions of colleagues at MIT, Ken Stevens's work has been of great help in linguistic phonetics.

2:00

4pSC2. A model of speech motor control and supporting data: Influences of quantal effects. Joseph S. Perkell (MIT-RLE, Rm. 36-591, 50 Vassar St., Cambridge, MA 02139) and Frank H. Guenther (Boston Univ., Boston, MA 02215)

A model of speech motor control is presented, along with examples of supporting data. In the model, segmental speech movements result from a process which plans a trajectory that passes through a sequence of phoneme-specific auditory goals. Some auditory goals are constrained by quantal relations between the vocal-tract area function and the resulting acoustics, as Stevens has proposed, and some are defined by quantal relations between motor commands and the area function, called biomechanical saturation effects. Both types of quantal relations make it possible to produce sounds that have relatively stable acoustic properties using somewhat imprecise motor commands. Therefore, quantal effects influence motor control strategies of individual speakers. They also influence sound patterns of languages, in that they help to define sounds such as the vowels /i/ and /a/ that occur frequently among languages of the world. Although there are other influences on speech motor control mechanisms and sound patterns, quantal effects are considered to be one of the defining characteristics of spoken language. [Work supported by NIDCD, NIH.]

\section{$2: 30$}

4pSC3. The "noisy" speech chain. Abeer Alwan (Dept. of Elec. Eng., UCLA, 405 Hilgard Ave., Los Angeles, CA 90095)

Kenneth Stevens' seminal contributions to the field of acoustic phonetics are far reaching and have important implications for modeling normal and disordered speech production and perception. His studies of the relations between underlying discrete linguistic features and the analog worlds of articulation, sound, and hearing have influenced a generation of speech researchers, including myself. Our studies of speech perception in noise are inspired by the work of Ken and his colleagues on uncovering the acoustic and perceptual correlates of linguistic features. I will report on experiments which examined acoustic and perceptual (in noise) cues for the 
place of articulation and voicing features for syllable-initial plosive and fricative consonants. Results are analyzed in terms of voicing, vowel context, manner of articulation, place of articulation, shape of the noise masker, and gender of the talker. One of the interesting findings is that certain attributes which cue a feature acoustically and perceptually in quiet conditions (such as the VOT for the feature voicing) do not correlate well with the perceptual robustness of the feature in noise.

3:00

4pSC4. Theoretical approaches to speech perception. Patricia K. Kuhl (Dept. of Speech and Hearing Sci. and Ctr. for Mind, Brain, and Learning, Univ. of Washington, Seattle, WA 98195)

Theoretical approaches to speech perception have shifted dramatically during the last 50 years. Early theories, such as Alvin Liberman's "motor Theory," focused on the psychological mechanisms underlying speech perception rather than listeners' analysis of the acoustic signal. In the 1970's, Ken Stevens' work on the acoustic cues for phonetic categories produced a very different view, an "invariance theory," that turned theoretical attention to the acoustic signal and the detailed phonetic information that listeners gleaned from its analysis. The last decade has seen another theoretical shift. Strongly influenced by developmental studies, new theories (e.g., Kuhl's native language magnet model, or NLM) combine the sensory and psychological approaches, arguing that listeners both perform a detailed analysis of language input and psychologically represent that information in a complex way that is unique to each language. The presentation will discuss these shifts and the kinds of data now necessary to produce the next theoretical advance. [Work supported by NICHD HD37954 and Human Frontiers RG0159 to PKK.]

\section{3:30-3:45 Break}

$3: 45$

4pSC5. Human communication with information systems. James Flanagan (Ctr. for Adv. Information Processing, Rutgers Univ., Piscataway, NJ 08854)

As networked information systems increase in complexity, natural modes of communication assume a greater role in the design of human-machine interfaces. Modalities of sound, sight, and touch are largely favored by humans, with conversational interaction being a central medium for information exchange. The distinguished research career of Professor Kenneth N. Stevens, celebrated in this special session on speech communication, has contributed directly to the basic understanding that supports current capabilities in voice interaction. While as yet primitive in comparison to human versatility, the technologies for sound, in concert with those for sight and touch, can be prudently applied to transcend the functionalities of traditional mouse and keyboard. This report describes a research effort in simultaneous utilization and fusion of sound, sight, and touch signaling in a networked collaborative environment. The conversational interaction depends upon automatic speech recognition, speech synthesis, and "hands-free" sound capture. Automatic face recognition, visual gesture, and eyetracking serve the sight modality. A force-feedback tactile glove provides manual gesture and grasp of virtual objects. And, intelligent software agents fuse and manage the multiple modes to develop a context-aware estimate of user intent. An experimental system utilizing these interface modes is applied to crisis management, and is demonstrated by videotape. [Components of this research were supported by National Science Foundation Contract Nos. IRI-96-18854 (STIMULATE) and IIS-98-72995 (KDI), DARPA Contract No. N66001-96-C-8510 (DISCIPLE), the Intel Corporation, Texas Instruments, and the Rutgers Center for Advanced Information Processing.]

\section{4:15}

4pSC6. Conversational Interfaces: Today and Tomorrow. Victor Zue (MIT Lab. for Computer Sci., 545 Technology Square, Cambridge, MA 02139)

For more than a decade, the Spoken Language Systems Group at the MIT Laboratory for Computer Science (with members made up of four generations of Ken Steven's disciples), have been conducting research leading to the development of conversational interfaces that enable users to access and manage information using spoken dialogue. Our research has been heavily influenced by Ken's teaching and research, ranging from acoustic landmark detection, acoustic-phonetic modeling, hierarchical phonological modeling using subword units such as syllables and features, lexical access strategies, and speech synthesis. While we are far from being able to develop systems capable of carrying on unrestricted conversations with users, we are beginning to field systems that have restricted domain expertise. For example, users can now speak to computers over the telephone, and receive weather, flight arrivaldeparture, and traffic information. One can even conduct air travel planning, and receive flight and fare information, all by simply speaking to the computer. This talk will highlight some of our recent work, and speculate on the future. [Work supported by DARPA and NSF.]

4:45

4pSC7. Retrieving distinctive features and segments from variable acoustic cues. Kenneth N. Stevens (Res. Lab of Elec. and Dept. of EECS, MIT, 50 Vassar St., Cambridge, MA 02139) and Samuel J. Keyser (Dept. of Ling., MIT, Cambridge, MA 02139)

It is assumed that words are stored in memory as sequences of bundles of distinctive features. Accessing words from the acoustic signal requires that these features be identified and organized into segments. Variability is a major obstacle to this process. Accounting for this variability requires a clear understanding of how variability arises when an utterance is generated by a speaker. It is proposed that variability arises from the operation of two principles: (1) Conservation: some of the defining gestures for a feature in a given segment may overlap with gestures from adjacent segments, causing weakening or masking of certain acoustic cues. (2) Enhancement: depending on the segmental and prosodic environment in which a feature occurs, additional gestures not directly specified by the 
feature may be introduced to enhance its perceptual salience. Conservation and enhancement provide a basis on which to select acoustic cues that reflect the gestures that produced them. Selection of acoustic cues for the features of consonant place, voicing, and nasality will be described and it will be shown that combinations of these cues can lead to feature identification with low error rate. [Work supported in part by grants from NIDCD.]

\title{
Session 4pSP
}

\section{Signal Processing in Acoustics, Underwater Acoustics and Speech Communication: Blind Deconvolution and Source Separation in Acoustics}

\author{
Leon H. Sibul, Chair \\ Acoustics Department, Pennsylvania State University, P.O. Box 30, State College, Pennsylvania 16804
}

Chair's Introduction-12:55

Invited Papers

1:00

4pSP1. Overview of blind signal separation, part I: Criteria and algorithms. Scott Douglas (Dept. of Elec. Eng., Southern Methodist Univ., Dallas, TX 75275 )

In blind signal separation, multiple independent source signals are separated from multiple linear mixtures of these signals without specific knowledge of either the source signal characteristics or the mixing conditions. This talk provides an introduction to the blind signal separation task. Three different problem formulations—signal separation of instantaneous mixtures, signal separation of convolutive mixtures, and multichannel blind deconvolution - are described, and their similarities and differences are highlighted. An overview of both information-theoretic and contrast-based separation criteria is then given. Natural gradient optimization procedures, when combined with such criteria, yield simple and useful blind signal separation algorithms. An example of speech separation of real room recordings illustrates the capabilities and limitations of one such approach.

\section{1:30}

4pSP2. Overview of blind signal separation, part II: Structures and applications. Kari Torkkola (Motorola Labs, 2100 E. Elliot Rd., MD EL 508, Tempe, AZ 85284)

The focus of this talk is a class of blind signal separation procedures, blind separation of convolutive mixing of signals. The emphasis is on acoustic signals and methods applicable thereof, and their capabilities and limitations. At least two possible taxonomies of separation methods are possible. These are based on how to parametrize the separating structure, and on the criteria used for separation. The former will be the emphasis of the presentation. Different criteria to solve the problem are only briefly touched upon. Possible application areas are enumerated, especially in acoustics. Two exemplary areas are audio signal enhancement in the presence of disturbing noise, and machine vibration analysis. Some issues on why it is not straightforward to move blind separation techniques into real world applications are elaborated. Limitations in the audio setup are discussed that make unconstrained BSS an extremely hard (if indeed solvable) problem. Some further questions are posed and directions for further research are presented.

\section{2:00}

4pSP3. Realistic application of acoustic blind source separation. Lucas Parra (Sarnoff Corp., CN5300, Princeton, NJ 08543)

Blind source separation in an acoustic environment is a challenging problem. The reverberation times in realistic rooms require long separation filters. They have to be estimated in very short times as the room responses change drastically with user location. In addition, convolutive blind separation has a number of indeterminations that make the identification of filter coefficients difficult. Decorrelation of changing cross-power spectra of nonstationary signal has been proposed as a separation criterion by various authors. We have presented in particular a frequency domain on-line algorithm that seeks to decorrelate cross-power spectra estimated over multiple times. We will report the performance of the algorithm in a realistic environment. The results will be compared to more conventional adaptive beamforming algorithms. Furthermore, speech recognition results for a command and control application with a distant talking user will be presented.

\section{2:30}

4pSP4. Blind source separation and blind deconvolution in experimental acoustics. Michael J. Roan and Josh Erling (The Appl. Res. Lab., Penn State Univ., P.O. Box 30, University Park, PA 16802, mjr110@psu.edu)

In active noise control, vibration control, and noise analysis, observed signals are typically a mixture of many sources, possible convolved with an unknown filtering function. A new area of research in the area of blind source separation and blind devonvolution (which has had application on the area of wireless communications) has promising application in noise and vibration control, 
radar/sonar, and noise analysis. The area of research exploits information theoretic criteria (rather than higher statistics) for blind source separation (BSS) and blind deconvolution (BDC). In this paper, several algorithms for BSS and BDC are presented. These algorithms are applied to experimental data. Experimental work in the areas of BSS has been with the separation speech signals from several speakers, and BDC work has been with speech signals convolved with artificial filters. It is shown through experimental results that several real acoustic filters (i.e., driven duct, driven duct with expansion chamber, and driven duct with Helmholtz resonator sidebranch) can be deconvolved. It is shown that application of the learned inverse filtering functions to the measured time series removes the effect of the filtering function. Finally methods for applying the algorithms for noise source identification, noise/vibration control, and noise analysis are presented.

3:00

4pSP5. Deriving optimal codes for speech and natural sounds using generalized independent component analysis. Michael S. Lewicki (Computer Sci. Dept. and Ctr. for the Neural Basis of Cognition, Carnegie Mellon Univ., 4400 Fifth Ave., Pittsburgh, PA 15213)

A Bayesian method for inferring an optimal basis is applied to the problem of finding efficient codes for natural sounds. The key to the algorithm is multivariate non-Gaussian density estimation, which is an equivalent independent component analysis when the form of the marginal density is fixed. An important advantage of the probabilistic framework is that it provides a method for comparing the coding efficiency of different bases objectively, and the derived codes can be shown to have better coding efficiency compared to traditional Fourier and wavelet bases. It also provides a method for Bayesian signal denoising and filling in of missing samples using a basis that is optimized to the structures in the data. When this framework is applied to deriving efficient codes of speech and natural sounds, the codes share many of the coding properties of the cochlear nerve. Time-frequency analysis shows that it is possible to derive both Fourier-like and wavelet-like representations by deriving efficient codes for different classes of natural sounds.

3:30-3:45 Break

\section{Contributed Papers}

\section{$3: 45$}

4pSP6. Blind source separation of mixtures of speech signals with unknown propagation delays. Phillip De Leon and Yunsheng Ma (Klipsch School of Elec. \& Computer Eng., New Mexico State Univ., Box 30001, Dept. 3-0, Las Cruces, NM 88003-8001, pdeleon@nmsu.edu)

Blind source separation of mixtures of speech signals has received considerable attention in the research community over the last 2 years. One computationally efficient method employs a gradient search algorithm to maximize the kurtosis of the outputs thereby achieving separation of the source signals. While this method has reported excellent separation results (30-50-dB SIR), it assumes a simple linear mixing model. In the general case, convolutional mixing models are used, however, this is a rather difficult problem due to causality and stability restrictions on the inverse not to mention length requirements in the FIR approximation. Research results with the general problem are modest at best. In this paper, we extend the kurtosis maximization ideas for source separation to include delays in the mixing model to at least account for propagation delays from speakers to microphones. The algorithm is designed to first estimate the relative delays of the sources within each mixture using a standard autocorrelation technique. These delay estimates are then used in the kurtosis maximization algorithm where the separation matrix is now modified to include these delays. Simulation results (using the TIMIT speech corpus) generally indicate good separation quality $(10-20 \mathrm{~dB})$ with little additional computational overhead.

\section{4:00}

4pSP7. Blind separation of $L$ sources from $M$ mixtures of speech signals. Phillip De Leon and Yunsheng Ma (Klipsch School of Elec. \& Computer Eng., New Mexico State Univ., Box 30001, Dept. 3-0, Las Cruces, NM 88003-8001, pdeleon@nmsu.edu)

In many real-world applications of blind source separation, the number of mixture signals, $L$, available for analysis often differs from the number of sources, $M$, which may be present. In this paper, we extend a successful and efficient kurtosis maximization algorithm used in speech separation of two sources from two linear mixtures for use in problems with arbitrary numbers of sources and mixtures. We examine three cases: underdetermined $(M<L)$, critically determined $(M=L)$, and overdetermined $(M$ $>L$ ). In each of these cases, we present simulation results (using the TIMIT speech corpus) and discuss separation matrix initialization issues and observed algorithm limitations. We find that in the critically determined case, the algorithm performs well (20-40 dB SIR) at separating four sources from four mixtures. For the other cases, our results are mixed. In the overdetermined case (two sources, three mixtures), the algorithm performs well (20-40 dB SIR) and we find that the extra mixtures do not result in better SIR measurements. In the underdetermined case (three sources, two mixtures), we are able to separate out at least one source (sometimes two) with the other output signals each containing pairs of the remaining sources.

\section{4:15}

4pSP8. Robust speech recognition in adverse environments by separating speech and noise sources using JADE-ICA. Shubha Kadambe (HRL Labs., LLC, 3011 Malibu Canyon Rd., Malibu, CA 90265, skadambe@hrl.com)

Spoken dialogue information retrieval applications are the future trend for mobile users. The typical presence of background noise in mobile environments causes significant reduction in speech recognition accuracy unless the recognizers are trained explicitly for each environment. However, it is not practical to train recognizers for every environment. One solution to this problem is to use an array of microphones and then separate the speech signals from noise by applying Blind Source Separation (BSS) techniques. BSS based on Independent Component Analysis (ICA) is one of the efficient techniques to separate mixed signals and JADE-ICA is one of ICA's variants that is applicable for non-Gaussian signals. The JADE-ICA is applied here, since generally speech and noise are nonGaussian signals. An array of two microphones was considered and two signal sources: speech and in-vehicle noise were assumed. Recognition accuracy experiments were conducted on 1831 utterances from 1831 speakers after separating the speech signals from noise using the JADEICA. The word recognition accuracy for three conditions: clean speech, noisy speech $(\mathrm{SNR}=0 \mathrm{~dB})$ and noise separated speech are $91.9 \%, 45.2 \%$ and $91.9 \%$, respectively. This indicates that using the JADE-ICA to separate speech from noise results in the same recognition accuracy as that of clean speech. The details on JADE-ICA and simulations will be provided in the full paper. 
were presented by Anemüller at the joint ASA/EAA/DAGA meeting, Berlin, 1999. The algorithm is based on the fact that the frequency-specific envelopes of speech signals exhibit correlation across different frequencies. This feature can be used to solve the "permutation problem" of frequency-domain-based blind source separation algorithms. Furthermore, it leads to separation of good quality since it results in a high number of constraints which must be fulfilled by the unmixed signals. Results for the separation of speech signals are presented for different mixing scenarios, including real-room reverberant mixing.

The problem of blind separation of a convolutive mixture of speech signals is considered. Signal separation is performed in the frequency domain. The amplitude modulation decorrelation algorithm for convolutive blind source separation of speech is described, the first ideas of which

$4: 45-5: 15$

Panel Discussion

THURSDAY AFTERNOON, 7 DECEMBER 2000

YAWL ROOM, 2:00 TO 3:30 P.M.

\title{
Meeting of Accredited Standards Committee (ASC) S3 on Bioacoustics
}

\author{
R. F. Burkard, Chair S3 \\ Hearing Research Laboratory, State University. of New York at Buffalo, 215 Parker Hall, Buffalo, New York 14214 \\ J. Franks, Vice Chair S3 \\ Robert A. Taft Laboratories, 4676 Columbia Parkway, Mail Stop C27, Cincinnati, Ohio 45226 \\ P. D. Schomer, Chair, U.S. Technical Advisory Group (TAG) for ISO/TC 43, Acoustics \\ 2117 Robert Drive, Champaign, Illinois 61821
}

D. D. Reynolds, Chair, U.S. Technical Advisory Group (TAG) for ISO/TC 108/SC4, Human Exposure to Mechanical Vibration and Shock

3939 Briar Crest Court, Las Vegas, Nevada 89120

H. E. von Gierke, Vice Chair, U.S. Technical Advisory Group (TAG) for ISO/TC 43, Acoustics and ISO/TC 108/SC4, Human Exposure to Mechanical Vibration and Shock

1325 Meadow Lane, Yellow Springs, Ohio 45387

V. Nedzelnitsky, U.S. Technical Advisor (TA) for IEC/TC 29, Electroacoustics

National Institute of Standards and Technology (NIST), Sound Building, Room A147, 100 Bureau Drive, Stop 8221,

Gaithersburg, Maryland 20899-8221

Accredited Standards Committee S3 on Bioacoustics. The current status of standards under preparation will be discussed. In addition to those topics of interest, including hearing conservation, noise, dosimeters, hearing aids, etc., consideration will be given to new standards which might be needed over the next few years. Open discussion of Committee reports is encouraged.

Scope of S3: Standards, specifications, methods of measurement and test, and terminology in the fields of mechanical shock and physiological acoustics, including aspects of general acoustics, shock, and vibration which pertain to biological safety, tolerance, and comfort. 


\title{
Meeting of Accredited Standards Committee (ASC) S1 on Acoustics
}

\author{
G. S. K. Wong, Chair S1 \\ Institute for National Measurement Standards (INMS), National Research Council, Ottawa, Ontario K1A OR6, Canada \\ P. D. Schomer, Chair, U. S. Technical Advisory Group (TAG) for ISO/TC 43, Acoustics \\ 2117 Robert Drive, Champaign, Illinois 61821 \\ T. J. Kuemmel, Vice Chair S1 \\ Quest Electronics, 510 South Worthington, Oconomowoc, Wisconsin 53066 \\ H. E. von Gierke, Vice Chair, U.S. Technical Advisory Group (TAG) for ISO/TC 43, Acoustics \\ 1325 Meadow Lane, Yellow Springs, Ohio 45387 \\ V. Nedzelnitsky, U.S. Technical Advisor (TA) for IEC/TC 29, Electroacoustics \\ National Institute of Standards and Technology (NIST), Sound Building, Room A147, 100 Bureau Drive, Stop 8221, \\ Gaithersburg, Maryland 20899-8221
}

\begin{abstract}
Accredited Standards Committee S1 on Acoustics. Working group chairs will report on their preparation of standards on methods of measurement and testing, and terminology, in physical acoustics, electroacoustics, sonics, ultrasonics, and underwater sound. Work in progress includes measurement of noise sources, noise dosimeters, integrating sound-level meters, and revision and extension of sound level meter specifications. Open discussion of Committee reports is encouraged.
\end{abstract}

Scope of S1: Standards, specifications, methods of measurement and test and terminology in the field of physical acoustics including architectural acoustics, electroacoustics, sonics and ultrasonics, and underwater sound, but excluding those aspects which pertain to biological safety, tolerance and comfort. 